US Army Corps

of Engineers ${ }_{\circledast}$

Engineer Research and

Development Center

Strategic Environmental Research and Development Program

Develop Accurate Methods for Characterizing and Quantifying Cohesive Sediment Erosion under Combined Current-Wave Conditions

Project ER 1497

Joseph Z. Gailani, Jesse D. Roberts, Sean P. Kearney

September 2017 
The U.S. Army Engineer Research and Development Center (ERDC) solves the nation's toughest engineering and environmental challenges. ERDC develops innovative solutions in civil and military engineering, geospatial sciences, water resources, and environmental sciences for the Army, the Department of Defense, civilian agencies, and our nation's public good. Find out more at www.erdc.usace.army.mil.

To search for other technical reports published by ERDC, visit the ERDC online library at http://acwc.sdp.sirsi.net/client/default. 


\title{
Develop Accurate Methods for Characterizing and Quantifying Cohesive Sediment Erosion under Combined Current-Wave Conditions
}

\author{
Project ER 1497 \\ Joseph Z. Gailani \\ Coastal and Hydraulics Laboratory \\ U.S. Army Engineer Research and Development Center \\ 3909 Halls Ferry Road \\ Vicksburg, MS 39180-6199 \\ Jesse D. Roberts, Sean P. Kearney \\ Sandia National Laboratories \\ 1515 Eubank SE \\ Albuquerque, NM 87123
}

Final report

Approved for public release; distribution is unlimited.

Prepared for Strategic Environmental Research and Development Program (SERDP) 48000 Mark Center Drive, Suite 17D08

Alexandria, VA 22350-3605

Under Work Unit W74RDV51808301, “Develop Accurate Methods for Characterizing and Quantifying Cohesive Sediment Erosion under Combined Current-Wave Conditions" 


\section{Abstract}

Aquatic cohesive sediments are often the final receptor of contaminants released by Department of Defense (DoD) activities. In estuaries, harbors, and channels, fine sediments can be mobilized and transported by the combined action of both relatively steady currents (forced by river flow, tides, and wind) and by the larger unsteady bottom shear stresses associated with higher-frequency oscillatory wave motions (either wind- or vessel-generated waves). Methods to measure contaminated sediment stability and erosion as influenced by wave processes are critical to assessing contaminant fate and associated risk as well as evaluating remediation options. This research focused on calibrating and verifying the applicability of a prototype flume designed to measure erosion of finegrained cohesive sediment under combined unidirectional and superimposed oscillatory bottom shear stress. These are similar to the shear-stress conditions that exist in wave-current environments. This research will provide more accurate methods for assessing contaminated sediment stability for many DoD and Environmental Protection Agency managed contaminated sediment sites.

DISCLAIMER: The contents of this report are not to be used for advertising, publication, or promotional purposes. Citation of trade names does not constitute an official endorsement or approval of the use of such commercial products. All product names and trademarks cited are the property of their respective owners. The findings of this report are not to be construed as an official Department of the Army position unless so designated by other authorized documents. 


\section{Contents}

Figures and Tables...

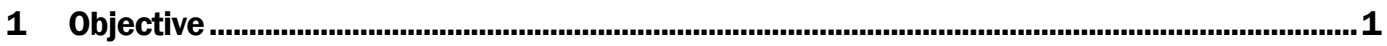

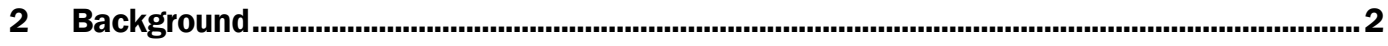

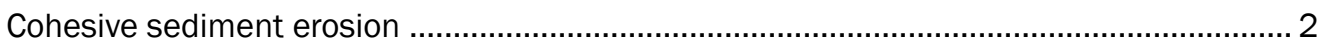

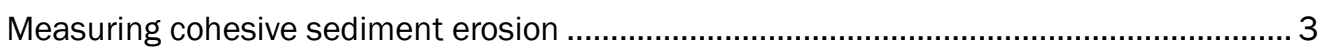

Cohesive sediment erosion algorithms .................................................................... 3

Cohesive sediment erosion under unsteady flow conditions ............................................. 4

Description of SEAWOLF flume...................................................................................... 5

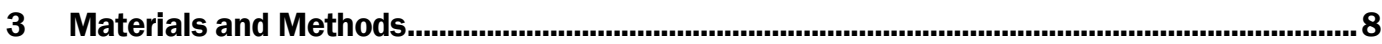

Task 1-Determine shear-stress time histories and turbulent structure within the enclosed SEAWOLF channel with smooth and rough walls under modulated flow ............. 9

Particle image velocimetry (PIV) operating principles...................................................... 10

PIV optical system and tracer particles.......................................................................... 11

Laser timing for unsteady flow measurements................................................................ 14

PIV image processing and spatial resolution................................................................... 15

Supporting theory for PIV-derived wall shear-stress measurements ................................... 15

Steady flow validation of PIV wall shear-stress measurements .......................................... 19

Phase averaging procedures for velocity and shear-stress results...................................... 20

Instantaneous flow field structure-two-point spatial correlations ...................................... 21

Instantaneous flow field structure-conditional velocity fields .............................................. 23

Task 2-Create data base of shear-stress time histories by demonstrating and applying newly developed optical sensing technology within SEAWOLF ............................ 24

MicroS, a micro-optical sublayer profilometer and shear-stress sensor ................................2 24

MiniLDV, a miniature laser doppler velocimeter (LDV) ..................................................... 26

Task 3-Demonstrate wave-current bottom shear-stress time histories representative of nature in SEAWOLF by manipulating flume and modulated flow

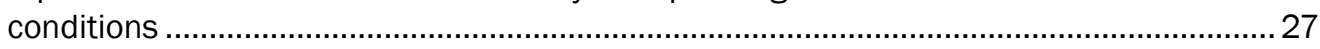

Task 4-Investigate uncertainty in shear-stress measurement techniques ......................28

4 Results and Accomplishments.

Task 1-Determine shear stress time histories and turbulent structure within the enclosed SEAWOLF channel with smooth and rough walls under modulated flow..... .29

Validation of PIV shear-stress measurements in a steady channel flow 
Cyclic modulation of wall shear stress and turbulent properties in oscillating

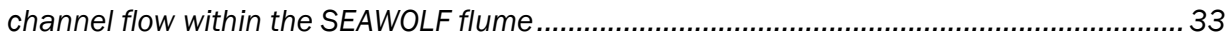

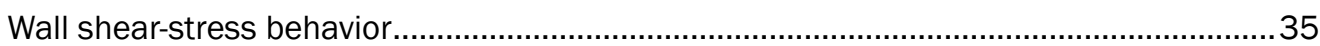

Visualizing the bulk flow in the intermittently turbulent flow (IT) regime (introduction to flow structure)

Turbulence structure in oscillating channel flow ..................................................................4 44

Task 2-Create data base of shear-stress time histories by demonstrating and applying newly developed optical sensing technology within SEAWOLF ...........................53

Optical shear-stress sensors demonstration ...................................................................... 53

Task 3-Demonstrate wave-current bottom shear-stress time histories representative of nature in SEAWOLF by manipulating flume and modulated flow

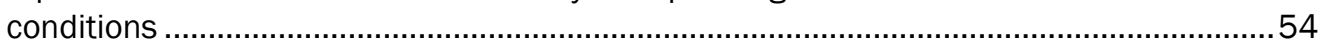

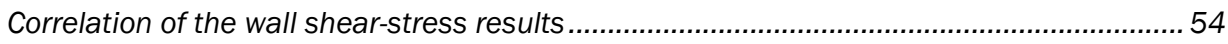

Acquisition of initial LDV data sets and comparison of LDV- and PIV-measured

shear stresses ................................................................................................................ 56

Measurement of instantaneous shear-stress fluctuations by LDV .....................................58

Parametric study of shear-stress waveforms generated by SEAWOLF ..................................60

Task 4-Investigate uncertainty in shear-stress measurement techniques .......................64

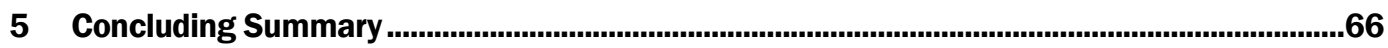

Task 1-Determine shear-stress time histories and turbulent structure within the enclosed SEAWOLF channel with smooth and rough walls under modulated flow

Cyclic modulation of wall shear-stress and turbulent properties in oscillating channel flow within the SEAWOLF flume. 66

Turbulence structure in oscillating channel flow .................................................................6 66

Task 2-Create data base of shear-stress time histories by demonstrating and applying newly developed optical sensing technology within SEAWOLF

Optical shear stress sensor demonstration.

Task 3-Demonstrate wave-current bottom shear-stress time histories representative of nature in SEAWOLF by manipulating flume and modulated flow conditions

Task 4-Investigate uncertainty in shear-stress measurement techniques 68

References

\section{Report Documentation Page}




\section{Figures and Tables}

\section{Figures}

Figure 1. SEAWOLF flume schematic. Three-tank system and flume that drives the unidirectional flow via a head difference $(\Delta h)$ between Tank $A$ and Tank $C$. Tank $B$ is overflow from Tanks $A$ and $C$, which is sent back to Tank $A$ via the pump.

Figure 2. Bottom view of circular insert hole in base plate where sample core is inserted. Rubber seals have been waterproofed with silicon and secured with nuts and hex bolts. 6

Figure 3. Dual pistons, which create the oscillatory flow in the flume..................................................

Figure 4. Conceptual depiction of the PIV system. ...........................................................................1

Figure 5. Schematic of the SEAWOLF test facility and PIV Instrumentation. ....................................12

Figure 6. Representative particle images at low- and high-magnification settings. ...........................13

Figure 7. Timing diagram for PIV measurements in oscillating flow.

Figure 8. High-magnification PIV results from a steady turbulent boundary layer: (a) streamwise velocity profiles expressed in terms of inner variables, (b) wall shear-stress data and comparison to Blasius correlation.

Figure 9. MicroS optical shear-stress sensor.

Figure 10. Conceptual operation of the miniLDV sensor.

Figure 11. Representative fits to near-wall velocity profiles recorded in an oscillating boundary-layer flow at $\operatorname{Re}_{\delta}=1220$. Data are shown in dimensional units and plotted on linear scales to illustrate the effectiveness of near-wall fitting. .

Figure 12. Phase-resolved shear-stress data for the test-matrix cases with $10 \mathrm{sec}$ wave period.

Figure 13. Phase-resolved shear-stress data for the $5 \mathrm{sec}$ wave period cases.

Figure 14. Phase modulation of the bulk velocity, wall shear stress, and channelaveraged turbulence.

Figure 15. Representative instantaneous velocity vector fields obtained during the acceleration phase of the half cycle at $\operatorname{Re}_{\delta}=1220$

Figure 16. Representative instantaneous velocity vector fields obtained during the deceleration phase of the half cycle at $\operatorname{Re}_{\delta}=1220$.

Figure 17. Near-wall mean velocity profiles at $R e_{\delta}=1220$ displayed in inner units. The viscous scales vary throughout the cycle and have been computed using phase-averaged values of the wall shear stress.

Figure 18. Phase-averaged modulation of the profiles, in outer units, of the turbulence quantities across the channel half height at $\operatorname{Re}_{\delta}=1220$, as acquired using lowmagnification PIV. Streamwise turbulence intensity, $u_{\mathrm{rms}} / U_{0}$; vertical turbulence intensity, $V_{r m s} / U_{0}$; Reynolds shear stress, $\left\langle u^{\prime} v^{\prime}\right\rangle / U_{0}{ }^{2}$; and rate of turbulence production, $\delta_{s} / U_{0}{ }^{3}$ $\left\langle u^{\prime} v^{\prime}\right\rangle \partial\langle u\rangle / \partial y$. Distance from the wall is normalized by the Stokes thickness, $\delta_{s}$.

Figure 19. Phase-averaged modulation of bulk velocity (top); wall skin friction (middle); inplane turbulent kinetic energy and turbulence production integrated across the channel height (bottom).

Figure 20. Instantaneous velocity vector measurements during the acceleration portion of the half cycle. 
Figure 21. Instantaneous velocity vector measurements during the deceleration portion of the half cycle.

Figure 22. Two-point spatial correlation of the streamwise velocity fluctuations at 10 different phase angles over the first half cycle. The event location, $y_{\text {ref, }}$ is located at $y / H=$ 0.137 .

Figure 23. Two-point spatial correlation of the wall-normal velocity fluctuations at 10 different phase angles over the first half cycle. The event location, $y_{\text {ref, }}$ is located at $y / H=$ 0.137.

Figure 24. Linear stochastic estimates of the expected velocity field given a $\lambda_{c i}$ event at $y_{\text {ref }}$ $/ H=0.137$ and $x / H=0$.

Figure 25. Measured (symbols) and modeled (solid line) LDV shear-stress response in steady channel flow.

Figure 26. Comparison of LDV- and PIV-measured shear stresses for 5 sec SEAWOLF wave period.

Figure 27. Comparison of LDV- and PIV-measured shear stresses for 10 sec SEAWOLF wave period.

Figure 28. Instantaneous shear-stress wave form measured using LDV in SEAWOLF.

Figure 29. Representative LDV shear-stress data sets for two different flow rate conditions at $10 \mathrm{sec}$ wave periods.

Figure 30. Phase-averaged data for operating condition 40_10_open_off.

Figure 31. Cycle minimum and maximum phase-averaged shear stresses correlated with their corresponding minimum and maximum phase-averaged flow rates.

Figure A1. Shear-stress results for operating condition 50_5_45_on. ............................................73

Figure A2. Shear-stress results for operating condition 50_5_60_on. ............................................73

Figure A3. Shear-stress results for operating condition 50_5_open_off. ............................................ 74

Figure A4. Shear-stress results for operating condition 50_5_45_off. ............................................... 74

Figure A5. Shear-stress results for operating condition 50_5_60_off. ...............................................75

Figure A6. Shear-stress results for operating condition 50_10_open_on.........................................75

Figure A7. Shear-stress results for operating condition 50_10_45_on................................................76

Figure A8. Shear-stress results for operating condition 50_10_open_off..........................................76

Figure A9. Shear-stress results for operating condition 50_10_45_off................................................77

Figure A10. Shear-stress results for operating condition 50_10_60_off. .........................................77

Figure A11. Shear-stress results for operating condition 40_10_45_off. ..........................................78

Figure A12. Shear-stress results for operating condition 40_10_45_on..........................................78

Figure A13. Shear-stress results for operating condition 40_10_60_on............................................79

Figure A14. Shear-stress results for operating condition 40_10_open_off.........................................79

Figure A15. Shear-stress results for operating condition 40_10_open_on........................................80

Figure A16. Shear-stress results for operating condition 40_5_open_on. ..........................................80

Figure A17. Shear-stress results for operating condition 40_5_45_on.............................................81

Figure A18. Shear-stress results for operating condition 40_5_60_on............................................81

Figure A19. Shear-stress results for operating condition 40_5_open_off..........................................82

Figure A20. Shear-stress results for operating condition 40_5_45_off.............................................82

Figure A21. Shear-stress results for operating condition 40_5_60_off.............................................83 
Figure A22. Shear-stress results for operating condition 50_5_open_on........................................83

\section{Tables}

Table 1. Summary of experimental test matrix. Actual metered flow rates are shown, which may differ slightly than the target flow rates in some cases....................................................31

Table 2. Summary of wall shear-stress results. ................................................................................

Table 3. Summary of peak phase-averaged flow and shear-stress data in SEAWOLF. ......................61 


\section{Preface}

This study was conducted for the Strategic Environmental Research and Development Program (SERDP), Work Unit W74RDV51808301, "Develop Accurate Methods for Characterizing and Quantifying Cohesive Sediment Erosion under Combined Current-Wave Conditions.” The technical monitor was Dr. Andrea Leeson of SERDP.

The work was performed by the Coastal Processes Branch (HF-C) of the Flood and Storm Protection Division, U.S. Army Engineer Research and Development Center, Coastal and Hydraulics Laboratory (ERDC-CHL). At the time of publication, Mr. Mark Gravens was Chief, CEERD-HF-C; Dr. Cary A. Talbot was Chief, CEERD-HF; and Mr. W. Jeff Lillycrop, CEERD-HT, was the Technical Director for Navigation. The Deputy Director of CHL was Mr. Jeffrey R. Eckstein, and the Director was Mr. José E. Sánchez.

At the time of publication of this report, COL Bryan S. Green was the Commander of ERDC, and Dr. David W. Pittman was the Director. 


\section{Acronyms}

$\begin{array}{ll}\text { DL } & \text { disturbed laminar flow } \\ \text { DoD } & \text { Department of Defense } \\ \text { EPA } & \text { Environmental Protection Agency } \\ \text { ERDC } & \text { U.S. Army Engineer Research and Development Center } \\ \text { FY } & \text { Federal Fiscal Year (1 October to 30 September) } \\ \text { GPM } & \text { gallons per minute flow } \\ \text { IT } & \text { intermittently turbulent flow } \\ \text { L } & \text { laminar flow } \\ \text { PI } & \text { principal investigator } \\ \text { PIV } & \text { particle image velocimetry } \\ \text { PTV } & \text { particle tracking velocimetry } \\ \text { SEAWOLF } & \begin{array}{l}\text { Sediment Erosion Actuated by Wave Oscillations and Linear } \\ \text { Flow }\end{array} \\ \text { SERDP } & \begin{array}{l}\text { Strategic Environmental Research and Development } \\ \text { Program }\end{array} \\ \text { UCSB } & \text { Sandia National Labs } \\ & \text { University of California at Santa Barbara } \\ & \end{array}$




\section{Unit Conversion Factors}

\begin{tabular}{|l|c|l|}
\hline Multiply & By & To Obtain \\
\hline degrees Fahrenheit & $($ F-32)/1.8 & degrees Celsius \\
\hline feet & 0.3048 & meters \\
\hline gallons (U.S. liquid) & $3.785412 \mathrm{E}-03$ & cubic meters \\
\hline inches & 0.0254 & meters \\
\hline inch-pounds (force) & 0.1129848 & newton meters \\
\hline microns & $1.0 \mathrm{E}-06$ & meters \\
\hline pints (U.S. liquid) & 0.473176 & liters \\
\hline pounds (force) & 4.448222 & newtons \\
\hline pounds (force) per square foot & 47.88026 & pascals \\
\hline yards & 0.9144 & meters \\
\hline
\end{tabular}




\section{Executive Summary}

\section{Objective}

The proposed research goal is to develop laboratory methods to reliably measure and predict erosion of contaminated fine-grained sediments under bottom shear-stress conditions that represent combined wave and current action. The shear-stress conditions for erosion measurements will replicate those expected in the field (nonbreaking wave, shallow water, estuarine, harbor, and coastal environments). The specific objective of this project is to calibrate and validate an innovative mobile erosion flume designed to measure cohesive sediment erosion under combined wavecurrent conditions.

\section{Background}

Aquatic cohesive sediments are often the final receptor of contaminants released by Department of Defense (DoD) activities. In estuaries, harbors, and channels, fine sediments can be mobilized and transported by the combined action of both relatively steady currents (forced by river flow, tides, and wind) and to an even greater extent by the larger, unsteady bottom shear stresses associated with higher-frequency oscillatory wave motions (either wind- or vessel-generated waves). Methods to measure contaminated sediment stability and erosion as influenced by wave processes are critical to assessing contaminant fate and associated risk as well as evaluating remediation options.

\section{Materials and methods}

This research focused on calibrating and verifying the applicability of a prototype flume designed to measure erosion of fine-grained cohesive sediment under combined unidirectional and superimposed oscillatory bottom shear stress. These are similar to the shear-stress conditions that exist in wave-current environments. The new flume is based on the design of a well-documented, frequently utilized flume for measuring cohesive sediment erosion under unidirectional bottom shear stress representing steady current (Roberts et al. 1998; McNeil et al. 1996). Preliminary results using the prototype flume (Sediment Erosion Actuated by Wave Oscillations and Linear Flow [SEAWOLF]) for the combined unidirectional and oscillatory flow have been published (Jepsen et al. 2002). 
Bottom shear stress under wave/current conditions has been studied extensively in the field and in large-scale laboratory wave flumes (Grant and Madsen 1979; Dibajnia and Watanabe 1992; Wikramanayake and Madsen 1994). The time history of shear stress that the sediment bed experiences under these combined conditions is therefore well understood in theory, and algorithms exist that accurately predict shear stress (Bijker 1971; Swart 1976). The SEAWOLF flume does not replicate the wave/current hydrodynamic conditions but rather replicates only the bottom shear stress that causes erosion. Free surface waves creating high-bottom shear cannot be developed in a field-deployable device at reasonable cost. The interior of the flume channel must be instrumented to demonstrate that bottom shear stress over the sediment surface in the flume is equivalent to specified wavecurrent conditions. The first step in demonstrating flume validity to wavecurrent conditions is to demonstrate that bottom shear stress over the sediment surface in the SEAWOLF flume replicates shear stress due to combined wave-currents. To demonstrate this, two instruments have been incorporated into the flume, one for measuring both near-bed and full-field flow structure (including velocity profile) and the second that simply measures near-bed velocity profile from which shear stress can be derived. The first system is a novel high-magnification, particle-based velocimetry system that utilizes particle-image velocimetry (PIV) and particle-tracking velocimetry (PTV) algorithms to resolve micro- and macro-scale flow structure as well as the velocity profile to within $7 \mu \mathrm{m}$ of the test section wall. Classical velocimetry systems are typically deployed in lower-energy, steady-flow, free-surface environments. Implementation within a closedchannel, high-shear, rapidly time-varying environment is a new application for these devices and one of the most challenging research aspects of this project. Issues related to time scale, turbulence, and secondary circulation under which these instruments have not been tested had to be analyzed.

The second system, the miniLDV, is a self-contained and permanently aligned laser Doppler velocimeter. The miniLDV probe contains a laser, miniature beam shaping optics, receiving optics, and a detection system. The size of the probe volume (measurement domain) is $\sim 25 \mu \mathrm{m}$ by $50 \mu \mathrm{m}$ by $150 \mu \mathrm{m}$. The system is mated with a computer-controlled linear traversing system allowing for resolution of near-bed velocity profiles.

Based upon the very interesting results from the hydrodynamic data collection activities, and with the Strategic Environmental Research and Development Program (SERDP) review panel approval, the path forward 
changed in 2008 to focus exclusively on hydrodynamic calibration and validation. Verification is required to prove that the bottom shear stresses created in the flume represent bottom shear stresses under naturally occurring wave-current conditions. Demonstrating flume applicability to wave-current environments requires designing standard operating procedures for SEAWOLF flume operation and collecting sufficient comparative laboratory data to prove SEAWOLF shear-stress replication methodology.

The addition of the miniLDV sensor will bring significant added benefit to the project, providing a coordination/comparison of several state-of-theart instruments never before tested in this type of environment. This rapidly varying, enclosed, high-shear environment is required not only for this project but for all cost-effective, high-energy sediment erosion experiment methods. Cost-effective methods will require replicating shear stress instead of hydrodynamic conditions. Therefore, the benefits of the shear-stress validation and procedure comparison will greatly benefit the larger sediment transport research community.

\section{Results}

Two major hydrodynamic studies are presented in this document. First, PIV was used to study the cyclic modulation of the wall shear-stress and turbulence properties of an oscillating channel flow. The PIV instrument employed utilized a dynamically adjusted delay between the laser pulses to accommodate the wide variations in velocity encountered in the oscillating flow. Both high- and low-magnification digital PIV recordings were obtained to reveal the near-wall boundary layer structure and wall shear stress, as well as the full-field turbulence throughout the channel. Presented are wall shear-stress and global turbulence data for Stokesthickness Reynolds numbers of $\operatorname{Re}_{\delta}=1220,2033$, and 2875. The results reveal a fully developed turbulent state, relaminarization, and an explosive transition back to turbulence. The flow is examined in detail for the case at $\operatorname{Re}_{\delta}=1220$, where instantaneous PIV realizations at low magnification reveal the structure of the flow during relaminarization and transition back to turbulence. High-magnification PIV results are used to reveal the phase modulation of the mean velocity profiles in the viscous sublayer and logarithmic layers through the half cycle and quantitative profiles of inplane Reynolds stresses and turbulence production are presented. To our knowledge, this is the first PIV investigation of this canonical unsteady 
turbulent channel flow, and these results represent a needed contribution to the limited turbulence data that exists for unsteady wall flows.

Second, the structure of turbulence in an oscillating channel flow with nearsinusoidal fluctuations in bulk velocity is investigated. Phase-locked particle-image velocimetry data in the streamwise/wall-normal plane are interrogated to reveal the phase-modulation of two-point velocity correlation functions and of linear stochastic estimates of the velocity fluctuation field given the presence of a vortex in the logarithmic region of the boundary layer. The results reveal the periodic modulation of turbulence structure between large-scale residual disturbances, relaminarization during periods of strong acceleration, and a quasi-steady flow with evidence of hairpin vortices that is established late in the acceleration phase and persists through much of the deceleration period. Again, to the best of the authors' knowledge, this is the first structural information for an oscillating flow derived from direct velocity measurements.

Also in 2008, a newly developed commercial miniLDV sensor was identified for potential application within the SEAWOLF. While reliable, PIV-derived measurements of shear-stress time histories offer limited amounts of data. Characterization of a single wave-current frequency and amplitude operating condition generally requires a full day of testing and an additional 1-2 days for analysis of the PIV data for the extraction of wall shear-stress histories. For the purposes of obtaining large parametric data sets that vary in frequency, amplitude, and bed-roughness conditions, a more rapid shearstress measurement must be incorporated. In previous years, a floating element mechanical shear stress sensor was tested that could be calibrated to a shear-stress value. These mechanical sensors have been applied for testing in SEAWOLF with mixed results; the sensors responded to timevarying shear stresses but displayed weak signal levels that were easily corrupted by electrical noise and suffered from time-dependent drift in sensor voltage, which corrupted the measurement as well.

As an alternative to mechanical shear-stress measurements, two newly developed commercial optical sensor technologies were tested, the microS and miniLDV, which (1) offer calibration-free measurements, (2) improved signal-to-noise characteristics, and (3) have been proven to work in harsh and dirty environments. The miniLDV significantly outperformed the microS in initial testing, and therefore it will be implemented within the SEAWOLF facility. 


\section{Benefit}

The proposed research will provide more accurate methods for assessing contaminated sediment stability for many DoD and Environmental Protection Agency managed contaminated sediment sites. The hydrodynamic validation exercises will produce stand-alone products that will benefit multiple other sediment transport projects. The final methods developed for measuring and predicting contaminated sediment erosion under wave-current conditions will be peer reviewed, cost effective, and standardized. Such methods do not presently exist, adding significant uncertainty in predicting contaminant fate and associated risk in shallowwater, coastal, estuarine, and harbor environments. The proposed research will develop methods to reduce uncertainty that presently complicates risk assessment and evaluation of remediation options.

\section{Transition plan}

There is a strong need for capabilities to measure erosion rates for buried contaminated sediment under wave-current conditions. A similar need previously existed for steady (current only) conditions. The transition of SEDflume (the current-only equivalent to the proposed wave-current flume) from research tool to frequently used field device for contaminated site evaluation was rapid (fewer than 3 years) due to the strong field-user need and well-documented, peer-reviewed flume validation. Similarly, the transition of the SEAWOLF flume from research tool to site evaluation device is expected to be rapid for two reasons: (1) the flume will be validated, and there are no alternative, cost-effective field devices for measuring erosion rates under these wave-current conditions and (2) there is a strong need in the remediation community for quantifying wavecurrent erosion of fine-grained sediments. An additional transition phase will involve incorporating erosion algorithms into existing predictive models such as the Advanced Hydraulics Model (AdH) and Long Term FATE (LTFATE) model (U.S. Army Engineer Waterways Experiment Station 1998). 


\section{Objective}

This Strategic Environmental Research and Development Program (SERDP) Project addresses SERDP Statement of Need CUSON-06-03, Assessment and Measurement of Processes Impacting the Fate and Transport of Contaminants in Sediments. The project specifically addresses the following high-priority needs outlined in the Statement of Need (SON):

Develop and evaluate site characterization tools to measure the rates of important sediment physical processes affecting the fate and transport of contaminants, and develop improved methods for incorporating uncertainty into measurements of fundamental fate and transport processes.

The overarching goal of Project ER 1497 is to develop methods to reliably measure and calculate/predict erosion of buried, contaminated, finegrained sediments under combined wave and current stresses. The methods will be developed for wave-current, shear-stress regimes typically encountered at contaminated sediment sites (shallow water, harbor, estuarine, and coastal environments). The measurement devices will be housed in a mobile trailer so that the measurements can be obtained at the site with minimum disturbance to the tested sediments.

This project includes the following technical objective:

Calibrate and validate an innovative mobile erosion flume designed to measure cohesive sediment erosion under combined wavecurrent shear-stress conditions. Devices have been previously developed and demonstrated for measuring cohesive sediment erosion and the variation with depth under unidirectional, steady current. This project involves developing and validating a similar flume for combined current-wave, unsteady flow conditions. The flume will be capable of measuring erosion variation with depth below the sediment/water interface so that the well-documented increase in resistance to erosion with consolidation is measured.

Achieving this technical objective will address the need for cost-effective, accurate methods for evaluating the potential for resuspension of buried contaminated sediments that can be incorporated into risk assessment framework. 


\section{Background}

Aquatic cohesive sediments are often the final receptor of contaminants released by Department of Defense (DoD) activities. These sediments have accumulated at numerous DoD sites. Understanding the stability and erosion of these fine sediments is critical to assessing contaminant fate and evaluating remediation options. In estuaries, bays, harbors, and channels, fine sediments can be mobilized and transported by the combined action of both relatively steady currents (forced by river flow, tides, and wind) and to an even greater extent by the larger unsteady bottom shear stresses associated with higher-frequency oscillatory wave motions (either wind- or vessel-generated waves). Contaminated site remediation is very expensive. Optimizing impact of remediation decisions requires defining conditions under which buried contaminated sediment may erode.

\section{Cohesive sediment erosion}

The majority of contaminated sediments are a mixture of sand, silt, and clay. These sediments are often referred to as cohesive because, unlike pure sand, bonding between particles influences sediment behavior. The complexity of cohesive sediment erosion is well documented (Gailani et al. 2001; Jepsen et al. 1997; Jepsen et al. 2002; McNeil et al. 1996; Roberts et al. 1998). The erosion characteristics of these sediments cannot be described using the well-established methods developed for sandy sediments, where erosion is primarily a function of grain size distribution and applied shear stress. Cohesive sediment erosion rates will be affected not only by grain size but also by bulk density, mineralogy, pore water chemistry, organic content, the presence of gas bubbles, and other factors. It is qualitatively understood that, depending on the conditions, small variations in one or more of these bulk properties may have an order of magnitude or more effect on erosion rates. However, insufficient data are available to quantify the effects of these properties on erosion rates. At present, when attempting to quantify erosion rates and conditions for a contaminated cohesive sediment bed, site-specific sediment erosion measurements are required to parameterize erosion equations. In addition, because bulk density will affect erosion rates, these rates will change with depth below the sediment-water interface even for well-mixed sediment, adding an additional level of complexity to erosion testing. Sitespecific measurement methodology exists for erosion under steady conditions; however, no validated devices or methods exist for measuring 
cohesive sediment erosion (and erosion variation with depth) under unsteady conditions similar to wave-current environments.

\section{Measuring cohesive sediment erosion}

The U.S. Army Engineer Research and Development Center (ERDC) and Sandia National Laboratories (SNL) have developed two portable field/laboratory flumes to measure cohesive sediment erosion rate variation with depth for steady flow conditions. The first flume is a variation of the University of California-Santa Barbara (UCSB) SEDflume (McNeil et al. 1996) for unidirectional, steady, high shear-stress flow. Prior to SEDflume development, only surficial sediment erosion could be reliably measured using portable erosion devices (Tsai and Lick 1986; Maa et al. 1993; Parchure and Mehta 1985). Erosion characteristics of underlying contaminated sediments measured using SEDflume are often quite different than the surficial sediment due to the effects of consolidation, biological activity, and other processes. A second flume, the ASSET flume (Jepsen et al. 2005; Roberts et al. 2003) that includes all the capabilities of SEDflume as well as the capability to separate bedload and suspended load transport of the eroded material, has also been developed. The flumes are designed for cost-effective assessment of cohesive sediment erosion rates. Flume erosion tests replicate the applied shear stress at the sediment bed (as opposed to flow velocity) and relate erosion to applied stress and sediment bulk properties. One major benefit of replicating shear stress instead of velocity regime is flume size. Shear stress can be replicated in a relatively small flume and with a small sediment sample size. Flumes that replicate flow regime must be significantly larger, require a larger sediment sample, and are generally not field deployable.

\section{Cohesive sediment erosion algorithms}

Algorithms based on relationships between erosion rate, applied shear stress, and sediment bulk properties ultimately allow for the predictive modeling of bottom sediment fate and transport and sediment bed change. Extensive SEDflume application was used to develop algorithms for cohesive sediment erosion variation with depth under unidirectional flow (Roberts et al. 1998). These algorithms have been demonstrated to be applicable to most natural cohesive sediments. These algorithms require site-specific parameterization developed through SEDflume analysis on the sediments of interest. Therefore, flume portability to the field is considered essential to quantifying erosion rates for existing sediment beds because 
transporting collected sediment cores for site-specific testing will result in an excessive disturbance that may alter erosion behavior. SEDflume and ASSET flume permit on-site assessment of erosion variation with depth for minimally disturbed sediment cores extracted from the contaminated bed and have been repeatedly demonstrated to accurately quantify high-shear, cohesive sediment erosion rate variation with depth through numerous laboratory and field applications (Jepsen et al. 1997; McNeil et al. 1996; Roberts et al. 1998). Flume data are used to develop predictive methods for analyzing the stability and/or erosion potential of cohesive sediment beds, including stratified beds. These predictions are used in risk assessment, site management, and assessment of remediation options for contaminated sediment sites (Borrowman et al. 2006). The Environmental Protection Agency (EPA), for example, utilizes SEDflume at most Superfund sites where buried contaminated sediment deposits are an issue. The flume is also commonly used in developing dredged material management plans and assessing the impact of cohesive sediment deposits on surrounding ecosystems.

\section{Cohesive sediment erosion under unsteady flow conditions}

Unidirectional, flow-induced, shear-stress conditions in the SEDflume and ASSET flume are a reasonable representation of shear-stress conditions in rivers or in deep water where wave influence on bottom shear stress is not significant. However, many contaminated sediment sites are in the shallow waters of lakes, reservoirs, harbors, large rivers, estuaries, or coastal regions where wave action influences near-bottom shear stresses. SEDflume and ASSET flume erosion rates measured under steady, unidirectional flow are not applicable to these environments. Therefore, additional flumes are required that represent the time-varying conditions experienced in waveinfluenced environments. The ERDC and SNL recently developed a prototype flume for measuring erosion under unidirectional flow with a superimposed oscillatory motion; these conditions represent shear conditions under a combined wave-current environment. The new flume is the previously defined SEAWOLF (Jepsen et al. 2002; Kearney et al. 2008). SEAWOLF is based on the proven SEDflume and ASSET flume designs. Like its predecessors, SEAWOLF mimics the shear-stress time history induced by given site conditions, as opposed to mimicking the actual hydrodynamics of the field, and will be a cost-effective, field-deployable method for measuring erosion rate variation with depth for minimally disturbed core samples. Algorithms based on sediment parameterization achieved by SEAWOLF flume testing will be developed in this research 
project so that contaminated sediment fate and transport can be predicatively modeled in combined wave-current environments. To this end, the new flume requires validation of applicability, similar to the multiyear effort required to validate SEDflume.

\section{Description of SEAWOLF flume}

SEAWOLF features a $2 \mathrm{~m}$ long, straight, clear polycarbonate flume channel (Figure 1). The channel has a false bottom at the center where a 10 $\mathrm{cm}$ diameter sediment core extracted directly from the field site (or created in the laboratory) is placed (Figure 2). Sediment cores can be up to $1 \mathrm{~m}$ in length below the initial sediment bed surface. As with SEDflume, the sediment core is eroded within the flume channel test section to quantify erosion rate. Sediment traps at each end of the flume channel remove sediments from the system such that core erosion in the test section is not influenced by sediment-laden water or bedload from previously eroded material.

Figure 1. SEAWOLF flume schematic. Three-tank system and flume that drives the unidirectional flow via a head difference $(\Delta h)$ between Tank $A$ and Tank $C$. Tank $B$ is overflow from Tanks $A$ and $C$, which is sent back to Tank A via the pump.

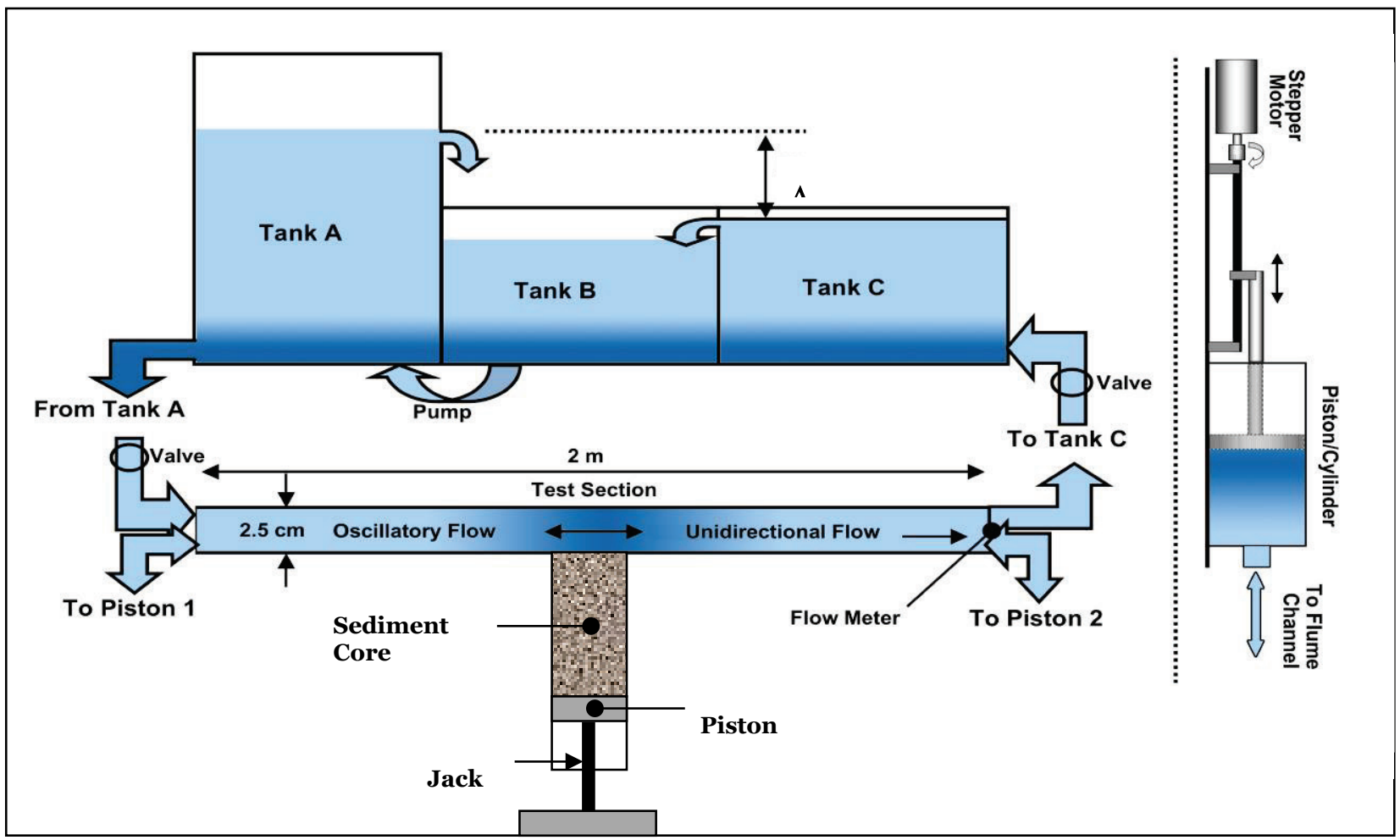


Figure 2. Bottom view of circular insert hole in base plate where sample core is inserted. Rubber seals have been waterproofed with silicon and secured with nuts and hex bolts.

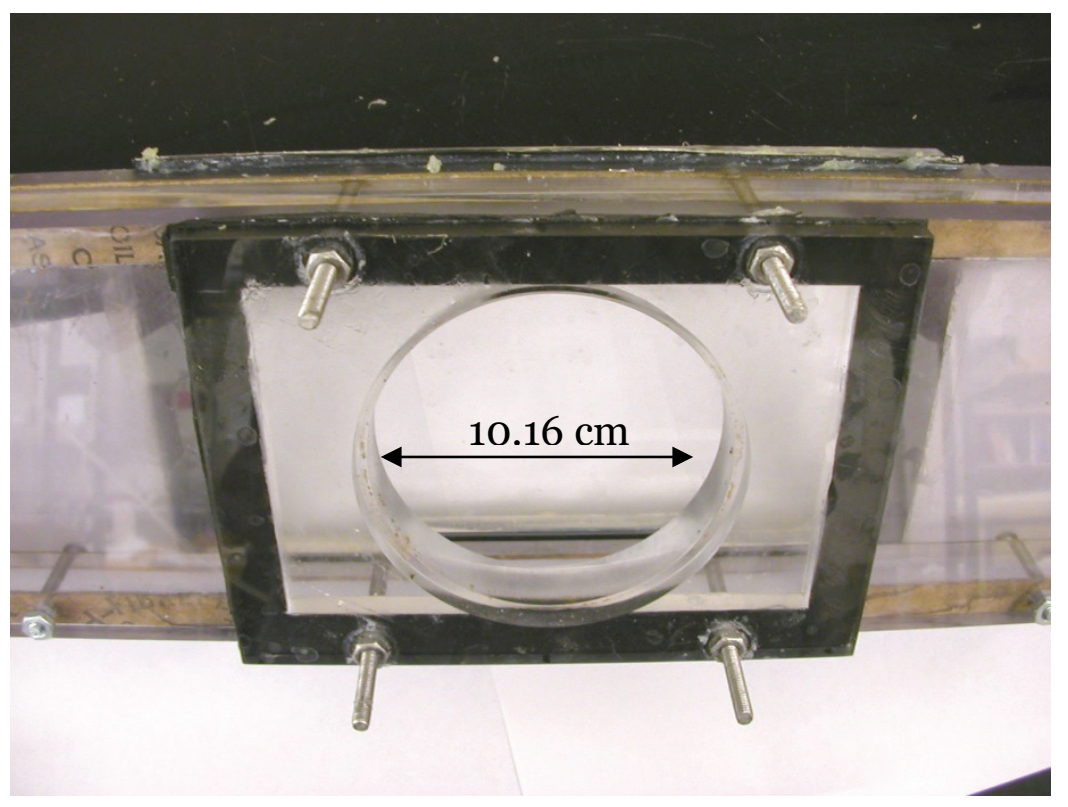

Tanks A and C are connected to opposite ends of the flume channel test section. Water is pumped from Tank B to Tank A to maintain the desired head in Tank A. The head in Tank A is greater than the head in Tank C. This head difference, $\Delta h$, which drives the unidirectional flow in the SEAWOLF test section, can be adjusted between each erosion test to represent user-specified mean current velocities. Both Tank A and Tank C overflow into Tank B to maintain constant $\Delta h$ during an erosion test. A magnetic flow meter attached directly to one end of the channel provides real-time measurements of flow conditions.

Two pistons (Figure 3) working in tandem (Figure 1) generate an oscillatory flow over the test section that represents wave-induced orbital velocities at the sediment bed. A computer, stepper motor, and linear ballscrew arrangement control the piston strokes that govern the velocity time history and period of the oscillatory flow. In addition, valves at each end of the channel connecting to Tank $\mathrm{A}$ or Tank $\mathrm{C}$ are used to control both the unidirectional flow rates and the backflow into the tanks from the oscillatory flow. Piston movement and head difference are preprogrammed by the operator before each erosion test. 
Figure 3. Dual pistons, which create the oscillatory flow in the flume.

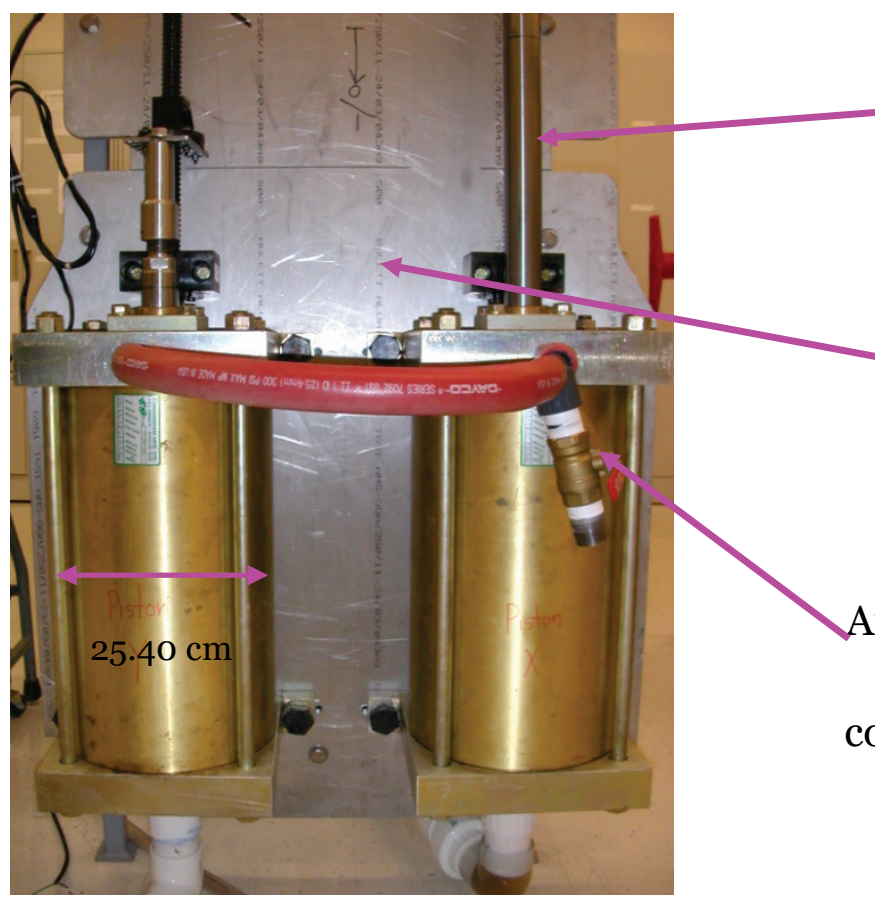

Drive Shaft

Aluminum Plate attached

to the tank support structure

Air Release Valve with flexible tubing connecting the pistons

The rectangular, cross-section test channel is $2 \mathrm{~m}$ long and $20 \mathrm{~mm}$ high $\times$ 105.4 $\mathrm{mm}$ wide (aspect ratio $=5 \cdot 5: 1$ ). All PIV measurements are made in a streamwise/wall-normal plane, which is located at the spanwise centerline at a streamwise location of $1 \mathrm{~m}$, or 52.5 channel heights, from the channel ends. BK-7 optical flats of $50.8 \mathrm{~mm}$ diameter are mounted flush with the test-section floor and ceiling at the measurement location to allow for passage of the Nd:YAG laser sheet used in the PIV measurements. The test-section ceiling, floor, and sidewalls are fabricated from $12.7 \mathrm{~mm}$ thick acrylic. A BK-7 optical flat is mounted in one of the sidewalls for PIV imaging at right angles to the laser sheet.

The test channel was sufficiently long to provide fully developed mean velocity profiles under steady flow conditions, as confirmed by PIVmeasured velocity profiles that conformed to the well-established law of the wall, and wall shear stresses that were generally within $10 \%$ of the Blasius correlation (White 1991) for fully developed turbulent wallbounded flow. The $2 \mathrm{~m}$ test-section length was limited by space constraints and was short enough that end effects were present, as fluid that began the forcing cycle at the measurement station left and re-entered the test section during the course of a forcing cycle. 


\section{Materials and Methods}

Based upon the very interesting results from the hydrodynamic data collection activities, and with the SERDP review panel's blessing, the path forward changed in 2008 to focus exclusively on hydrodynamic calibration and validation. Therefore, the objective evolved to include a series of experiments to validate the applicability of the prototype SEAWOLF flume to replicate shear-stress time histories in naturally occurring wave-current conditions. The change in path forward has necessitated a redevelopment of the milestones for this research project. The new tasks and subtasks are outlined below.

- Task 1 - Determine shear-stress time histories and turbulent structure within the enclosed SEAWOLF channel with smooth and rough walls under modulated flow

1.1 Assess the need to modify SEAWOLF Flume Channel to contain all oscillating flow and modify as necessary

1.2 PIV measurements in smooth-walled channel flows

1.3 PIV measurements in various rough-walled channel flows

- Task 2 - Create data base of shear-stress time histories by demonstrating and applying newly developed optical sensing technology within SEAWOLF

2.1 Identify and test capabilities of an optical shear-stress sensor within SEAWOLF

2.2 Implementation of optical shear-stress sensor in smooth-walled channel flow

2.3 Optical shear-stress measurements in rough-walled channel flow 2.4 Generate comprehensive database of shear-stress time histories in SEAWOLF using optical shear-sensor technology measurements

- Task 3 - Demonstrate wave-current bottom shear-stress time histories representative of nature in SEAWOLF by manipulating flume and modulated flow conditions

3.1 Assessment of existing knowledge of shear-stress time histories in naturally occurring bottom boundary layers 
3.2 Configure SEAWOLF Flume for replication of wave-current boundary layer shear stress

3.3 Replicate naturally occurring wave-current conditions in SEAWOLF Flume

- Task 4 - Investigate uncertainty in shear-stress measurement techniques

4.1 Assess uncertainty in PIV shear-stress measurements

4.2 Assess uncertainty of optical sensor shear-stress measurements

These tasks outline the specific objective of this research, which is to focus on quantifying and controlling time-varying bottom shear stress within the enclosed channel of the SEAWOLF for combined unidirectional and oscillatory flow (modulated flow). These combined flows are representative of near-bottom hydrodynamic conditions in a wave-current environment and are therefore representative of shear stress applied to the sediment bed. Velocity profile and turbulence length scale have not been satisfactorily measured in an enclosed channel for the purpose of quantifying wall (bed) shear stress in modulated flow. Therefore, it is imperative to measure phase-resolved turbulent flow structure and wall shear stress within a confined flume with modulated flow. Once measurement techniques have been demonstrated, the next step is to control the turbulent flow structure to construct a desired shear-stress time history that is relevant to nature by adjusting bottom roughness, flume structure, and flow parameters.

\section{Task 1-Determine shear-stress time histories and turbulent structure within the enclosed SEAWOLF channel with smooth and rough walls under modulated flow}

Erosion is generally quantified as a function of bottom shear stress. Shear stress is a function of the near-bottom velocity profile (not mean velocity) and sediment bed roughness. The complex, time-varying bottom shear stress in SEAWOLF had not been extensively measured or tested prior to Strategic environmental Research and Development Program (SERDP) funding. To accurately quantify wave-current induced erosion, the SEAWOLF bottom shear-stress time series within the flume must reflect the time series of shear stress due to a specified combined current-wave condition that occurs in nature. Currently, theory cannot describe time series shear stress within an enclosed channel subject to oscillating flow (theory can be and is used to relate flowrate to shear stress in SEDflume 
and the ASSET flume-no measurements were deemed necessary in these steady flow flumes). Shear-stress time history in the flume channel must be quantified through direct measurement of near-bottom velocity profile, leading to a calculation of bottom shear stresses. These measurements permit control of piston movement and head difference to replicate bottom shear-stress time histories generated during specified wavecurrent conditions.

\section{Particle image velocimetry (PIV) operating principles}

The PIV technique is described in picture form in Figure 4. With PIV, the flow of interest is seeded with particles that are both small and light enough to faithfully track the flow. The particles are illuminated using a planar sheet of pulsed laser light. The laser pulses must be short enough to effectively freeze the particle field, such that particle motion during the laser illumination is negligible. For this purpose, pulsed Nd:YAG lasers, that provide visible $532 \mathrm{~nm}$ (green) light with a short, $10 \mathrm{~ns}$ pulse duration, are typically used. Simple cylindrical and spherical lens elements are typically used to re-form the laser-light output into a thin sheet of light, which illuminates the tracer particles in the flow. For PIV measurement, two laser pulses are delivered in succession with a known, prescribed time delay, $\Delta t$, between the laser pulses. An interline transfer CCD camera is then used to record two digital particle images in rapid succession, with exposures which are timed such that each image frames one of the laser pulses. The particle-image pairs contain information about an instantaneous snapshot of the velocity field in the plane of the laser sheet. The digital images are broken down into 16-32 pixel square interrogation windows and the average displacement, $\Delta \mathbf{x}$, of the particles in each window during the time $\Delta t$ between the laser pulses is determined using statistical cross-correlation techniques. The velocity vector in each interrogation spot is then determined from

$$
\mathbf{u}=\Delta \mathbf{x} / \Delta t
$$


Figure 4. Conceptual depiction of the PIV system.

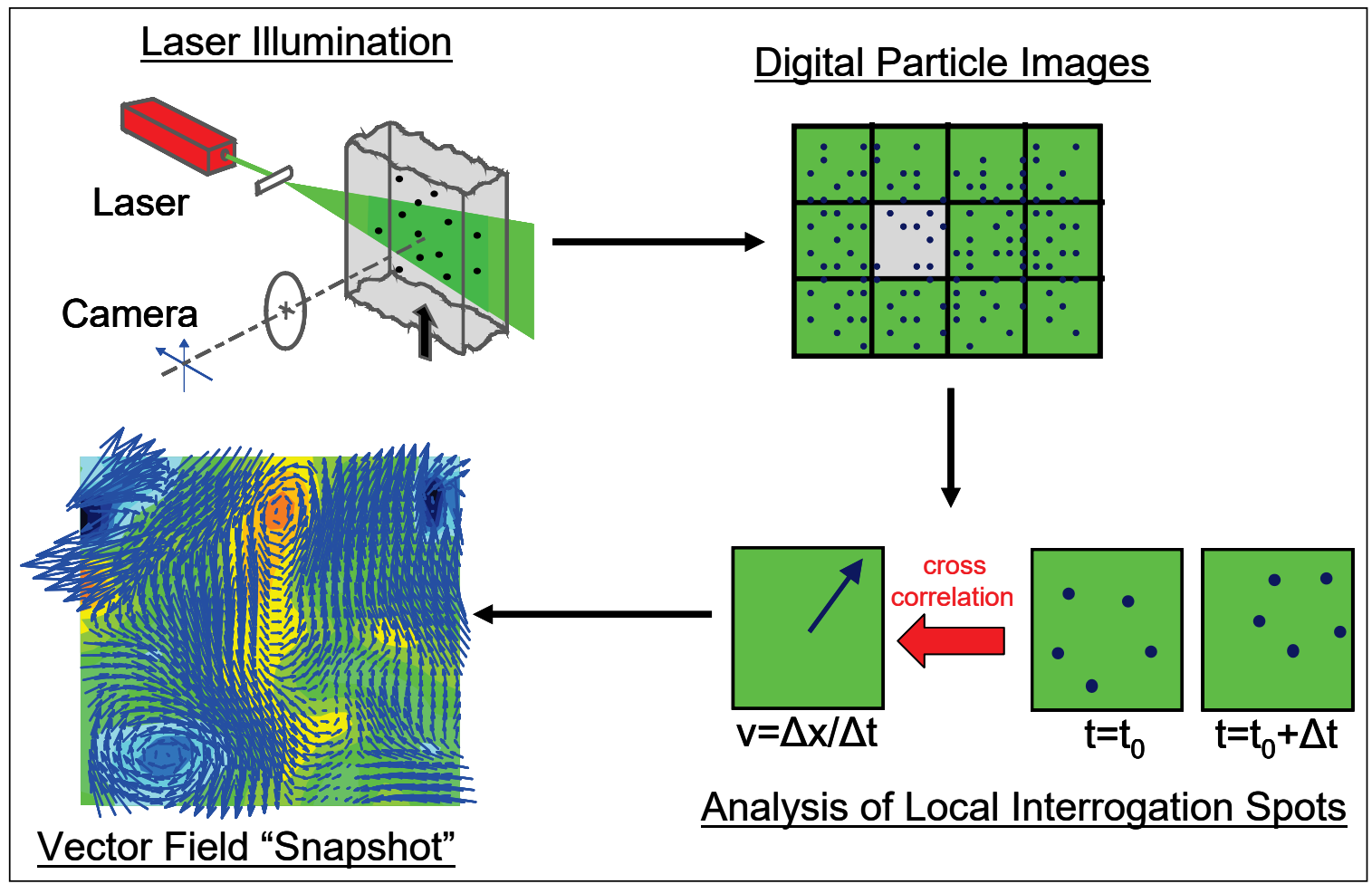

Since both the horizontal and vertical particle displacements of the tracer particles can be determined from the two-dimensional (2D) particle images, $\mathbf{u}$ is then a vector quantity containing the two components of the velocity field, $u$ and $v$ (horizontal and vertical), in the plane of the laser sheet. When this process is repeated at each interrogation spot in the images, 2D vector maps, such as those shown in Figure 4 are obtained. These maps reveal the instantaneous structure of a complex turbulent flow. Many such maps can then be statistically analyzed to extract the mean velocity field and the statistical fluctuations of the velocity which define the turbulent Reynolds stress tensor.

\section{PIV optical system and tracer particles}

A schematic of the PIV optical system is presented alongside the drawing of the SEAWOLF channel in Figure 5. A dual-cavity, frequency-doubled Nd:YAG laser (Continuum MiniLite) provides Q-switched laser pulses of 8 ns duration, with energies of $\sim 25 \mathrm{~mJ} /$ pulse at a wavelength of $532 \mathrm{~nm}$. Particle images were captured on an interline-transfer CCD camera (Kodak ES 1.0) with $1024 \times 1024$ pixel format and subsequently stored for crosscorrelation postprocessing. PIV experiments at two magnification settings were conducted: (1) a low-magnification (0.33:1) setting, attained using a 
$105 \mathrm{~mm}$ Nikkor lens at $f / 8$ aperture, was used to obtain PIV recordings with a field of view that spanned the full height of the channel and (2) a highmagnification $(\sim 3.45: 1)$ setting, using the same $105 \mathrm{~mm}$ lens with a $2 \times$ teleconverter and extension rings, to obtain a field of view which was nominally $2.5 \mathrm{~mm}$ square. The low-magnification PIV experiments allowed viewing of the large-scale behavior of the unsteady flow field while the highmagnification data sets permit resolution of the viscous sublayer (described in detail in subsequent sections) and obtain a direct measurement of the wall shear-stress modulation throughout the cycle.

Figure 5. Schematic of the SEAWOLF test facility and PIV Instrumentation.

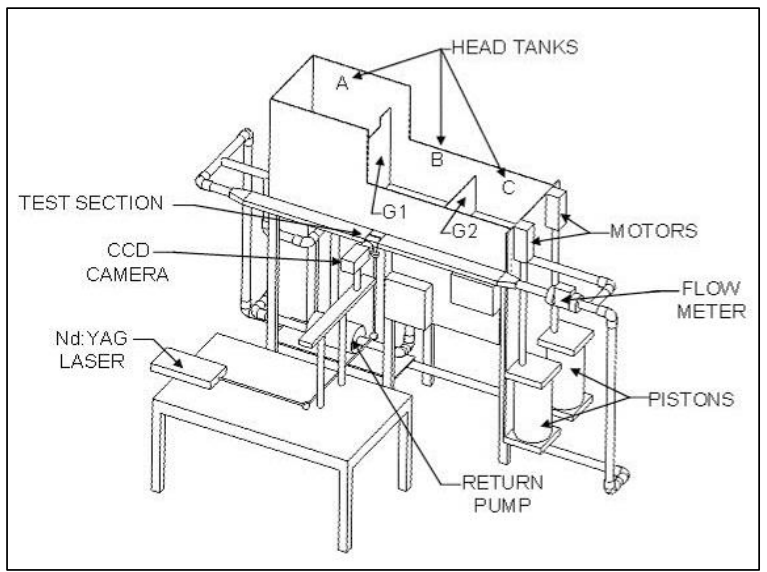

The flow was seeded with $1.03 \mathrm{~g} / \mathrm{cc}, 5-\mu \mathrm{m}$-diameter polyamide particles, which were nearly neutrally buoyant in water. The SEAWOLF facility was further contaminated by residual sediment particles from earlier testing, which were estimated to be as large as $35 \mu \mathrm{m}$, and could not be completely removed. The flow-tracking ability of both seed and contaminant particles was estimated from the particle Stokes number:

$$
S k=\frac{\rho_{p} d_{p}^{2}}{18 \mu} f_{c}
$$

where $\rho_{p}$ is the particle density, $d_{p}$ is the particle diameter, $\mu$ is the dynamic viscosity, and $f_{c}$ is a characteristic frequency of the flowfield that can be estimated from the rates of strain present in the flow. For a worstcase estimate, $f_{c}$ was computed using the viscous timescale, $t^{+}=v / u_{\tau}^{2}$, where $u_{\tau}$ is the friction velocity, and $v$ the kinematic viscosity, as $t^{+}$provides an estimate of the strain rate in the high-velocity-gradient region at the wall. The polyamide particles, as expected, yielded $S k$ values of order $10^{-3}$, 
compared to values of 0.1 to 0.7 for the sediment particles. These worstcase values were small enough to guarantee faithful particle tracking in most cases, with the exception of sediment contaminants in the very nearwall region and only at the high end of the shear stresses present in this study. As discussed in section IV, particle tracking does not impact the estimates of wall shear stress, which were validated using a steady turbulent channel flow as a test case.

Representative high- and low-magnification particle images are shown in Figure 6. The low-magnification images are of excellent quality and permitted extraction of high-resolution PIV results. Good particle-image density was observed in the high-magnification realizations, but the quality of the high-magnification results was degraded somewhat as the diffraction-limited spot size becomes comparable or better than the particle diameters, resulting in a greater disparity in particle-image size between seed and contaminant particles. No processing of the particle images was performed to differentiate between small and large particle images, and velocity data were necessarily obtained from both seed and contaminant particles.

Figure 6. Representative particle images at low- and high-magnification settings.

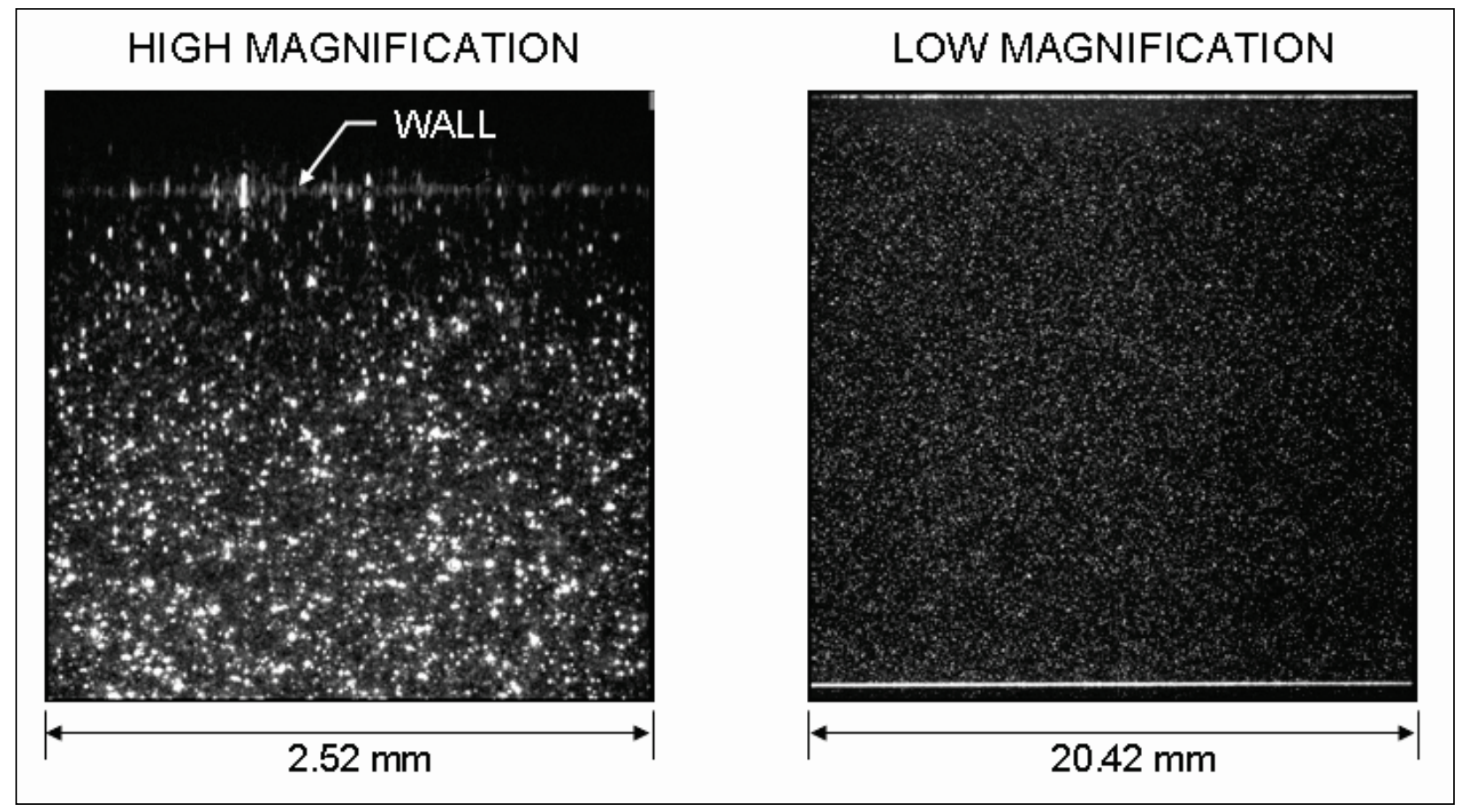




\section{Laser timing for unsteady flow measurements}

The large variation in bulk velocity through the cycle required a continuous varying of the time delay between the Nd:YAG laser pulses used for PIV. A timing diagram for the PIV measurements is provided in Figure 7 to illustrate this procedure. All timing pulses were generated using counter/ timer chips provided with commercial data-acquisition cards, controlled using a custom LABVIEW program. A fixed number of 40 preprogrammed laser-pulse delays were manually entered into a list in the LABVIEW software, which was coded to provide a set of 40 triggers per flow cycle, and then there was a wait until the next cycle was initiated by the SEAWOLF pistons. The start of the flow-forcing cycle was indicated by the transistortransistor logic (TTL) pulse (uppermost in Figure 5) from an optical switch, which was actuated at the end of travel of one of the pistons when the path between its light source and photodiode was broken by a metal tab that traveled with the piston shaft. Trigger pulses for the CCD camera and laser A (second and third pulse trains in Figure 7) were provided in uniform $T / 40$ increments, where $T$ is the period of the flow forcing cycle. The delay between laser A and laser B was then varied (fourth pulse train in Figure 7) using the preprogrammed list to the LABVIEW code, which cycled automatically through the sequence and then stood by until the beginning of the next forcing cycle was triggered by the optical switch. The trigger to the CCD camera was also used to acquire data from a volume flow meter and a thermocouple located near the entrance to the test section simultaneously with the PIV recordings. The variable delay between laser pulses was set to obtain a maximum streamwise displacement of nominally $10-15$ pixels in the particle-image pairs, which yields a dynamic velocity measurement range of 100-150:1 for the streamwise component and 10-20:1 for the vertical component, based on a commonly assumed (Raffel et al. 2002) \pm 0.1 pixel uncertainty in the location of the cross-correlation peak.

Figure 7. Timing diagram for PIV measurements in oscillating flow.

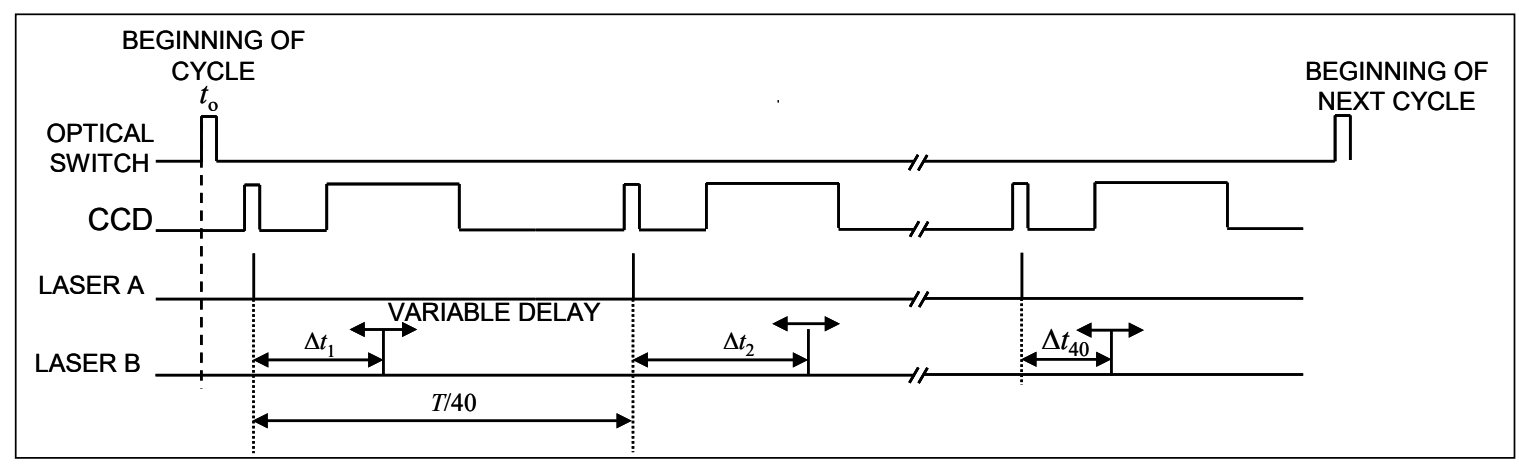




\section{PIV image processing and spatial resolution}

Our particle images were interrogated using cross-correlation algorithms implemented in the DaVis commercial software package (DaVis 2006). The procedure was initiated using a coarse $64 \times 64$ pixel interrogation-window size with no window offset, which was required since the direction of the streamwise velocity was not known a priori. The coarse field was liberally interpolated and smoothed to provide an estimate for window displacement in subsequent passes with finer resolution. The final PIV result was obtained using $16 \times 16$ pixel windows with 50\% spatial overlap. Outliers were removed from the vector fields, and holes in the final vector estimates were conservatively interpolated. The spatial resolution in the velocity vector data was $160 \mu \mathrm{m}$ and $19.6 \mu \mathrm{m}$ for the low- and high-magnification measurements, respectively. When scaled to inner variables, the corresponding in-plane spatial resolutions range from 1.7 to 13.1 viscous wall units for the low-magnification data and 0.2 to 1.6 wall units at highmagnification, for a measured range of wall shear stresses of 0.1 to $6 \mathrm{~Pa}$. The out-of-plane resolution in the data was estimated at 2 to 20 wall units, based on an assumed $200 \mu \mathrm{m}$ overlap of the laser sheets.

\section{Supporting theory for PIV-derived wall shear-stress measurements}

A key objective of this effort is the determination of wall shear stress by direct measurement of the gradient of the phase-resolved velocity profile at the wall, using the well-known constitutive relation:

$$
\tau_{w}=\mu \frac{d u}{d y}
$$

where $\tau_{w}$ is the wall shear stress, $\mu$ is the dynamic viscosity, and $d u / d y$ is the slope of the mean velocity profile at the wall, which is to be estimated from high-magnification PIV data. For this procedure to be valid, a sound justification is sought for a linear fit to the PIV-measured velocity data in some region close to the wall. The following analysis is a review of wellknown dimensional scaling laws for flow in the innermost portions of a boundary layer, very close to the wall, as presented by Hinze (1987) ${ }^{1}$. This analysis justifies the use of a linear representation of the velocity profile in the near-wall region, which makes PIV shear-stress determination possible.

\footnotetext{
${ }^{1}$ See section 7-5 of Hinze (1987) for a discussion of scaling in the inner portion of the boundary layer.
} 
Very close to the wall, the dynamics of the boundary layer flow are dominated by the momentum transfer at the wall, through the shear stress, given by Equation 3. If Equation 3 is divided by the density, $\rho$, a velocity scale that is dependent on the shear stress is obtained, which drives the flow in the near wall region:

$$
\frac{\tau_{w}}{\rho}=v \frac{d u}{d y}=u_{\tau}^{2}
$$

where $v$ is the kinematic viscosity and $u_{\tau}$ has units of velocity and is commonly referred to as the friction velocity, which is the appropriate velocity scale for the near-wall flow. Dimensional analysis of Equations 2 and 3 reveals that the near-wall velocity profile $u=u\left(u_{\tau}, v, y\right)$, which can be reduced to a functional relationship between dimensionless variables using the $\Pi$ theorem:

$$
\frac{u}{u_{\tau}}=f\left(\frac{u_{\tau} y}{v}\right)
$$

or

$$
u^{+}=f\left(y^{+}\right)
$$

Equation (6) is a functional form or scaling law, which holds in the socalled inner region of a boundary layer, very far from the boundary layer edge, channel centerline, or other boundary, so that the shear stress at the wall solely governs the flow dynamics. As a result, $u^{+}$and $y^{+}$are referred to as inner variables.

The form of the function $f\left(y^{+}\right)$will dictate the equation that is needed to fit the PIV data in the near-wall region. In the immediate vicinity of the wall, the no-slip condition requires that the velocity be near zero. Transport of momentum is dominated by the molecular collisions represented by the viscosity so that the velocity profile is entirely determined by Equation 3, which is integrated from the wall to a near-wall position, $y$, to obtain the following expression for the near-wall velocity profile:

$$
u(y)=\frac{\tau_{w}}{\rho v} y=u_{\tau} y^{+}
$$


which can be rearranged by dividing by $u_{\tau}$ to obtain

$$
u^{+}=y^{+} \text {for } y^{+}<5
$$

Equations 7 and 8 are valid in the very low-momentum region immediately adjacent to the wall, where viscous diffusion is the dominant force affecting the velocity profile. Experimentally, Equation $6 \mathrm{~b}$ has been shown to be valid for only a very thin region between the wall and $y^{+}=5$, beyond which turbulent motion begins to impact the transport in addition to purely viscous forces. This relationship reveals that the velocity profile in this viscous sublayer is linear and that a linear fit to PIV data measured very near the wall is justified for the determination of $d u / d y$ and the wall shear stress through Equation 3.

Proceeding farther from the wall, turbulent mixing just begins to impact the transport of momentum in the near-wall region and becomes comparable or even greater than the molecular viscous forces. The impact of this turbulent mixing manifests itself as an apparent time-averaged shear stress, which can be expressed in a form similar to Equation 3 using a turbulent or eddy viscosity, vt. The combined impact of the viscous and turbulent shear forces can then be written as

$$
\frac{\tau}{\rho}=\frac{\tau_{\text {viscous }}+\tau_{\text {turbulent }}}{\rho}=\left(v+v_{t}\right) \frac{d u}{d y}
$$

Experiments show that by a distance of approximately $y^{+}=30$, the turbulent mixing dominates and $v_{t}>>v$. Based on physical arguments (Akhavan et al. 1991) vt can be expressed as

$$
v_{t}=\kappa u_{\tau} y
$$

where $\kappa$ is a proportionality constant. If Equation 10 is substituted into Equation 7 and the turbulent-mixing-dominated case is considered, with $v t>>$, for the region for $y^{+}>30$, the following is obtained:

$$
\frac{\tau}{\rho}=u_{\tau}^{2}=\kappa u_{\tau} y \frac{d u}{d y}
$$

or in dimensionless quantities, 


$$
1=\kappa y^{+} \frac{d u^{+}}{d y^{+}}
$$

Equation 12 can be integrated to obtain the following functional form for the mixing-dominated region of the inner layer:

$$
u^{+}=\frac{1}{\kappa} \ln y^{+}+B \text { for } y^{+}>30
$$

For steady, wall-bounded turbulent flows, the constants have been determined experimentally to be $\kappa=0.41$ and $B \sim 5^{-6}$.

Equations 7 and 13 represent analytical forms for turbulent boundary layers. Since the dimensionless variables, $u^{+}$and $y^{+}$, in these expressions depend on the value of the wall shear stress, these equations can be used to fit the near-wall PIV data for the determination of the wall shear stress. The linear expression for the viscous sublayer in Equation 8 is valid for steady and unsteady, laminar and turbulent flows. Furthermore, Equation $6 \mathrm{~b}$ has no empirically determined constants and is thus a general way of determining shear stress from measured velocity profiles. The drawback to Equation 8is that the viscous sublayer is often very thin, and velocity measurements very close to the wall are extremely challenging. The logarithmic region for $y^{+}>30$ is simpler to access experimentally, and fits to the form of Equation 10 are often used for the measurement of wall shear stress in steady turbulent boundary layers (Clauser 1956; Kendall and Koochesfahani 2006). The derivation of Equation 10 presented here is for steady turbulent flows only, but Akhavan et al. (1991) has shown that the logarithmic form is also valid for unsteady turbulent boundary layers as well. The two main drawbacks to logarithmic fitting in unsteady flows are (1) the log law only applies under turbulent conditions, while the oscillating and pulsating flows considered here undergo periods of laminar and transitional flow, and (2) the constants $\kappa$ and $B$ in the log law are not well known for unsteady flows, and they can change throughout the forcing cycle, further making Equation 10 difficult to apply in unsteady flows.

Fitting of the viscous sublayer and use of Equation 3 was determined to be the best option for PIV-based shear-stress measurements in SEAWOLF, as this method was most general and likely to be the most accurate. With this in mind, the high-magnification PIV instrument previously described was constructed. For the $0-6$ Pa range of shear stresses observed in the SEAWOLF water flow, the thickness of the viscous sublayer varies from approximately 70 to $220 \mu \mathrm{m}$ so that any PIV measurement for wall-shear- 
stress determination must have very fine spatial resolution. The $19.6 \mu \mathrm{m}$ spatial resolution of the high-magnification system permits 3 to 11 points within the viscous sublayer, to which a linear fit can be performed for the estimation of the wall velocity gradient, and direct measurement of the wall shear stress through Equation 3. The fidelity of this approach was then verified with measurements in steady turbulent channel flows, as explained in the Task 1.2 subsection of the Results and Accomplishments section of this report.

\section{Steady flow validation of PIV wall shear-stress measurements}

A key objective of this effort is the determination of wall shear stress by direct measurement of the gradient of the phase-averaged velocity profile at the wall using the high-magnification PIV approach suggested by Oweis and Ceccio and employed in their work (Oweis et al. 2010). The 0.2 to 1.6 viscous-wall-unit resolution of the high-magnification PIV data enables probing of the viscous sublayer for the Reynolds numbers of interest here. Wall shear stress is calculated from Equation 3 by estimating the velocity gradient from the slope of a least-squares line fit to all mean velocity data with $y^{+}<5$. The effectiveness of this procedure was investigated using data from steady turbulent flows established in the SEAWOLF test section. The shear-stress data were then compared with redundant estimates of $\tau_{w}$ obtained from Clauser-type fits layers (Clauser 1956; Kendall and Koochesfahani 2006) to the velocity data in the logarithmic layer and from the empirical shear-stress correlation due to Blasius (White 1991):

$$
\frac{2 \tau_{w}}{\rho U^{2}}=0.0791 R e_{d}^{-1 / 4}
$$

where $\rho$ is the density and $U$ is the mass-mean or bulk velocity in the channel.

These steady-flow, high-magnification PIV results are shown in Figure 8. Mean velocity profiles were computed from ensemble and streamwise averaging of 500 statistically independent PIV realizations, as shown in the uppermost plot in Figure 8. The mean velocity data were normalized using inner length scales computed from fitting of the log layer. At all Reynolds numbers, the velocity profiles display good collapse to the universal curve, with some scatter observed in the results closer to the wall, which is indicative of uncertainty in wall location and small differences in the viscous scales computed using shear-stress values from log-layer and viscous-layer fitting. 
Figure 8. High-magnification PIV results from a steady turbulent boundary layer: (a) streamwise velocity profiles expressed in terms of inner variables, (b) wall shear-stress data and comparison to Blasius correlation.

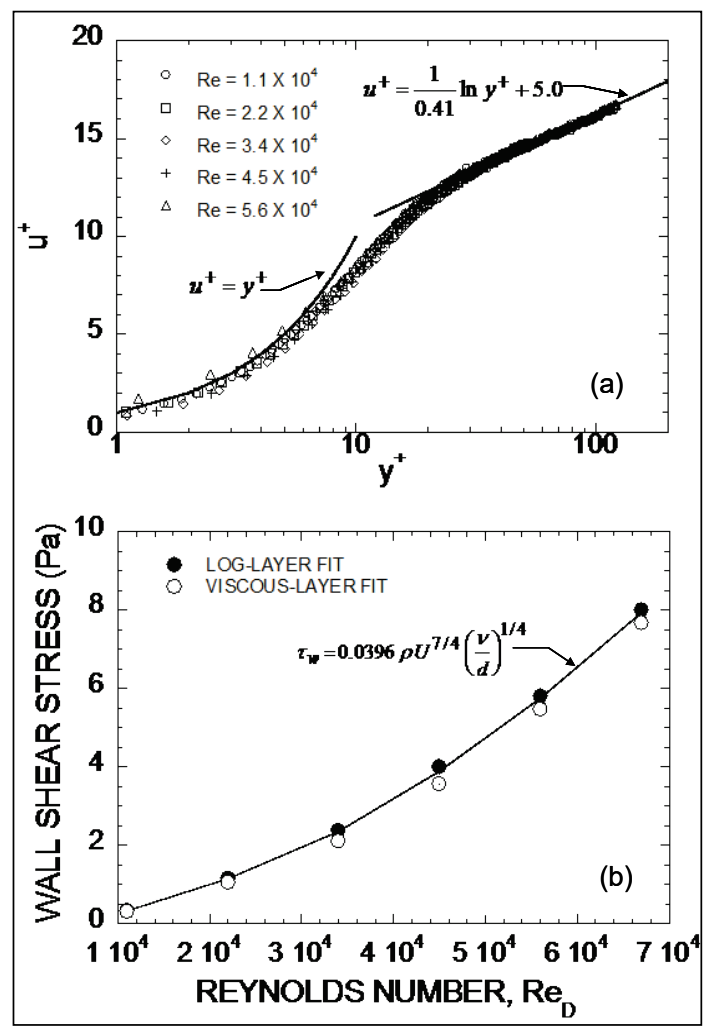

Wall shear-stress data from viscous and log-layer fits are provided as a function of Reynolds number in the lowermost plot in Figure 8. The experimental results are plotted alongside empirical values computed from Equation 14. Shear-stress estimates from linear fits to the velocity data in the viscous layer are within $-4 \%$ to $-13 \%$ of both Equation 6 and results from log-layer fitting, which was seen as reasonable level of accuracy for the measurements in the oscillating channel flow. Note that the outstanding agreement between the log-layer-fit results and Equation 11 is surely fortuitous and that none of the three shear-stress estimates in Figure 8 can be deemed as more reliable than any other. It is the $\sim 10 \%$ agreement among all three shear-stress estimates that gives confidence in the approach of fitting to the viscous-layer data.

\section{Phase averaging procedures for velocity and shear-stress results}

All velocity and turbulence quantities are presented in the context of a phase-averaging procedure that is common to the analysis of periodically 
unsteady turbulent flows (Akhavan et al. 1991; Jensen et al. 1989; Hino et al. 1983). In this analysis, any quantity, $q$ (representing a velocity component, shear stress, or other variable), can be expressed on an instantaneous basis in terms of a phase-locked average $\langle q\rangle$ and an instantaneous turbulent fluctuation, $q^{\prime}$ :

$$
q(\mathbf{x}, \tau)=\langle q\rangle(\mathbf{x}, \tau)+q^{\prime}(\mathbf{x}, t)
$$

where the functional dependences on position vector, $\mathbf{x}$; the periodnormalized time, $\tau=t / T$ (ranges from o to 1 over the course of the cycle); and an instantaneous time, $t$, are explicitly indicated. From discretely sampled data, the phase average simply provides a representation of the average cycle, and can be computed from

$$
\langle q\rangle\left(\mathbf{x}, \tau_{j}\right)=\frac{1}{N} \sum_{k=1}^{N} q\left[\mathbf{x}, t=\tau_{j} T+(k-1) T\right], j=1,2 \ldots . M
$$

where $N$ is the total number of cycles for which the data have been sampled-a minimum of 200 for the authors' data; $M=40$ is the number of samples per cycle; and $T$ is the period of the applied periodic forcing. The cyclic modulation of the turbulence statistics can then be computed using the phase-locked variance operation:

$$
\left\langle q^{\prime 2}\right\rangle\left(\mathbf{x}, \tau_{j}\right)=\frac{1}{N-1} \sum_{k=1}^{N}\left\{q\left[\mathbf{x}, t=\tau_{j} T+(k-1) T\right]-\langle q\rangle\left(\mathbf{x}, \tau_{j}\right)\right\}^{2}, j=1,2 \ldots . M
$$

One-dimensional profiles of phase-locked statistics were then computed by streamwise averaging the quantities defined in Equations 16 and 17. This procedure is valid since the channel flow is essentially fully developed so that gradients in the streamwise direction are expected to be small.

\section{Instantaneous flow field structure-two-point spatial correlations}

For the canonical problem of fully developed channel/pipe flow, analytical solutions exist for sinusoidal time variations in pressure gradient (Uchida 1956; Currie 1993) or volume flow rate (Muntges and Majdalani 2002; Ray et al. 2005), and the problem is well understood. In the turbulent regime, experimental data that describe the time-dependent evolution of the phase-locked, Reynolds-averaged flowfield statistics have been provided (Hino et al. 1983; Akhavan et al. 1991; Jensen et al. 1989), and these 
experimental data are complimented by more recent reports of LES/DNS simulations (Scotti and Piomelli 2001; Costamagna et al. 2003; Salon et al. 2007). Reynolds-averaged information is a useful descriptor of turbulent fields but does not always yield the needed insight into the fundamental dynamics of the flow that is given by the identification and description of spatially and temporally coherent structures, which have been extensively described in steady turbulent wall flows (Cantwell 1981; Adrian 2007) and have been found to contribute significantly to the production and maintenance of wall turbulence.

The description of the time evolution of coherent structures in periodically unsteady turbulent wall flows (hereafter referred to as oscillating flows) is less developed than for the steady case, but a picture is beginning to emerge based largely on the results of recent numerical simulations (Scotti and Piomelli 2001; Costamagna et al. 2003; Salon et al. 2007) and earlier flow visualization experiments (Fishler and Brodkey 1991; Sarpkaya 1993), which must be verified using a more quantitative experimental description based on direct and spatially correlated measurement of the velocity field. Earlier measurements in oscillating turbulent wall flows (Hino et al. 1983; Akhavan et al. 1991; Jensen et al. 1989) utilized point laser doppler velocimeter (LDV) data, from which structural and spatially correlated information cannot be extracted. This is believed to be the first PIV measurements in a canonical oscillating wall flow. Earlier research provided a Reynoldsaveraged description of the turbulent field along with a brief, qualitative presentation of the instantaneous 2D structure of the flow. A portion of the PIV data set is analyzed here to extract quantitative 2D structural and spatial correlation information, which can then be compared to the present view of turbulence structure in both oscillating and steady turbulent wall flows.

Statistical imprints of the instantaneous structure can be realized using two-point correlation functions of the velocity fluctuations. The evolution of two-point self-correlation functions of the streamwise $\left(R_{u u}\right)$ and wallnormal $(R v v)$ velocity fluctuation fields are discussed later in the text. Twopoint correlations were computed from phase-locked velocity fluctuation data using

$$
R_{i j}\left(r, y ; y_{o}, \theta\right)=\frac{\left\langle u_{i}\left(x, y_{o}\right) u_{j}(x+r, y)\right\rangle}{\sigma_{i}\left(y_{o}\right) \sigma_{j}(y)}
$$


where the $i, j$ subscripts refer to the streamwise and wall-normal directions, the $u k$ represent the velocity component fluctuations about their respective phase-locked means, the $\sigma k$ are the root mean square (rms) fluctuations of the $u k$, and the \langle\rangle brackets represent averaging over the $x$-coordinate and the ensemble of 200 phase-locked PIV realizations at phase angle $\theta$. The correlations were computed using a reference height, $y_{o}=0.137 \mathrm{H}$, which places the reference point in the log layer (when the flow is turbulent) for the majority of the forcing cycle.

\section{Instantaneous flow field structure-conditional velocity fields}

The two-point spatial correlation fields discussed provide a description of the mean size and orientation of the turbulent fluctuations but does not give further specifics on the details of the structure that can reveal the physical mechanisms responsible for the generation of correlated, large-scale features. In steady turbulent wall flows, there has been significant recent evidence to link boundary layer structure and the production/maintenance of Reynolds shear stress to the existence of organized groupings, or packets, of hairpin vortices (Adrian 2007). Several questions then arise regarding the existence and history of hairpin vortices in oscillating wall flows. Are hairpins present? When are they present? How do hairpins grow, develop, and dissipate in the presence of strong cyclic acceleration, deceleration, and relaminarization? To begin to address some of these questions, there is an extension of the analysis of Christensen and Adrian (Christensen and Adrian 2001), in which the expected value of the velocity fluctuation field, given the presence of a near-wall swirling strength event-which may or may not arise from hairpin-vortex motion-is computed. This conditionally averaged velocity field is estimated using linear stochastic estimation, which allows a computation of an unbiased estimate of the average based on conditional event data using unconditioned statistical correlations between velocity and swirling strength. The proper form of the linear estimate, as given by Christensen and Adrian, is

$$
\left\langle u_{j}(x+r, y) \mid \lambda_{c i}\left(x, y_{o}\right)\right\rangle \approx \frac{\left\langle\lambda_{c i}\left(x, y_{o}\right) u_{j}(x+r, y)\right\rangle}{\left\langle\lambda_{c i}\left(x, y_{o}\right) \lambda_{c i}\left(x, y_{o}\right)\right\rangle} \lambda_{c i}\left(x, y_{o}\right)
$$

From Equation 19, it is evident that the expected velocity given the condition of a $\lambda_{c i}$ event at height $y_{o}$ from the wall (note that the $i$ in $\lambda_{c i}$ is not an index in Equation 19) can be computed using the unconditional two-point correlation between $\lambda_{c i}$ and the velocity fluctuation components. 


\section{Task 2-Create data base of shear-stress time histories by demonstrating and applying newly developed optical sensing technology within SEAWOLF}

Identified this year were several newly developed commercial optical sensors for potential rapid shear-stress measurement applications within the SEAWOLF. While reliable, PIV-derived measurements of shear-stress time histories offer limited amounts of data. Characterization of a single wave-current frequency and amplitude operating condition generally requires a full day of testing and an additional 1-2 days for analysis of the PIV data for the extraction of wall shear-stress histories, which, to date, has created a limitation of shear-stress results for only eight frequency/ amplitude combinations. For the purposes of obtaining large parametric data sets that vary frequency, amplitude, and bed-roughness conditions, a more direct shear-stress measurement must be incorporated. Floatingelement measurements of shear stress, based on load-cell sensors, provide a direct mechanical force measurement that can be calibrated to a shearstress value. These mechanical sensors have been applied for testing in SEAWOLF with mixed results; the sensors responded to time-varying shear stresses but displayed weak signal levels that were easily corrupted by electrical noise and suffered from time-dependent drift in sensor voltage, which corrupted the measurement as well.

As an alternative to mechanical shear-stress measurements, there was an implementation of a newly developed commercial optical sensor technology that (1) offers calibration-free measurements, (2) improved signal-to-noise characteristics, and (3) has been proven to work in harsh and dirty environments. Two candidate commercial sensor technologies from Measurement Science Enterprise, Inc., Pasadena, CA, were demonstrated. They were the microS (Fourguette et al. 2004) and the miniLDV, summarized here.

\section{Micros, a micro-optical sublayer profilometer and shear-stress sensor}

An illustration of the microS sensor platform is provided in Figure 9. The sensor contains a miniaturized diode laser source and diffractive optics that project a coherent fringe pattern into the near-wall flow. The flow is seeded with particles (or sediment from erosion testing) that scatter light from the fringe pattern that is sensed by a fiber-coupled detector that images a measurement volume located $75^{-100} \mu \mathrm{m}$ from the bed surface, within the viscous sublayer where the velocity profile is known to be linear. The probe laser and detector element are fully integrated into the compact sensor housing (Figure 9). 


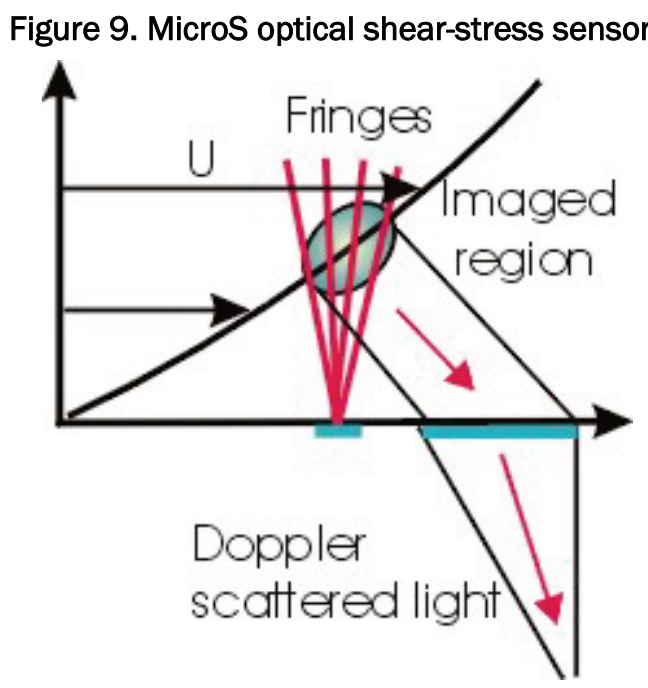

The local fringe separation, $\delta$, was designed to be linear with the distance from the sensor, $y$, given by $\delta=k y$, where $k$ is the fringe divergence rate along the normal to the sensor surface. As seed particles flow through the linearly diverging fringes, they scatter light with a frequency, $f$, which is proportional to the near-wall velocity and inversely proportional to the fringe separation. The velocity of the seed particle is then $u=f \delta$. The frequency simply multiplied by the fringe divergence yields

$$
\frac{\mathrm{u}}{\mathrm{y}}=\mathrm{fk}
$$

which is equal to the wall velocity gradient, provided that the probe volume is located within the viscous sublayer. The bed shear stress is then calculated from

$$
\tau=\mu \frac{d u}{d y}=\mu \frac{u}{y}=\mu f k
$$

where $\mu$ is the viscosity of water. The signal conditioning and processing required for the diverging fringe optical sensor is identical to that used for a standard laser-Doppler velocimetry and is supplied with the sensor in an integrated package.

This commercial sensor can be easily mounted flush with any bed surface inside an enclosed channel facility for parametric measurements of wall shear-stress time histories with rapid turnaround. It is easy to use, durable, and provides reliable data in the presence of fouling of its 
windows by seed particles and sediment. The manufacturer has applied the sensor for measurements in ocean and field environments where it was proven to be robust.

\section{MiniLDV, a miniature laser doppler velocimeter (LDV)}

Most LDVs in the industry are table-top systems that include the probe, an argon-ion laser, fiber manipulators for laser alignment, Bragg cells, and receiving and processing enclosures. Getting results quickly requires a user with experience in beam alignment. The miniLDV replaces all these components with a probe and two small electronics boxes, decreasing the sensor system's overall size as well as its power consumption. Since all the sensitive optics are enclosed within the probe, the user does not need to spend hours setting up the sensor, leaving more time to perform data collection.

The miniLDV system consists of a transceiver probe, driver electronics, bandpass filter, and BP-miniLDV burst processor acquisition hardware and software. The miniLDV is a standalone system meaning that no other lasers or optics are required. For use it is only necessary to place the electronics at a convenient distance from the acquisition computer and place the probe at the desired location. The burst processor software collects data, moves the probe on the electronic traverse, and presents flow statistics. The process of measuring a profile can be automated with the software by dictating the number and location of points from which to collect data.

Conceptually, an LDV system consists of highly coherent light split into two beams and sent through a transmitter that crosses the beams in the probe volume to create a fixed interference pattern (Figure 10). The aberrations of the optics must be low so that the fringes of the pattern are straight throughout the probe volume. The receiver consists of light collection optics and an ultra-fast-response photodiode. When a particle passes through the probe volume, it passes through regions of destructive interference (darkness) and constructive interference (brightness) that the receiver sees as a sinusoidal wave of intensity. Knowing the fringe separation (known from calibration of the given transmitter), the frequency of the sinusoid is a measure of the speed of the particle through the probe volume. 
Figure 10. Conceptual operation of the miniLDV sensor.

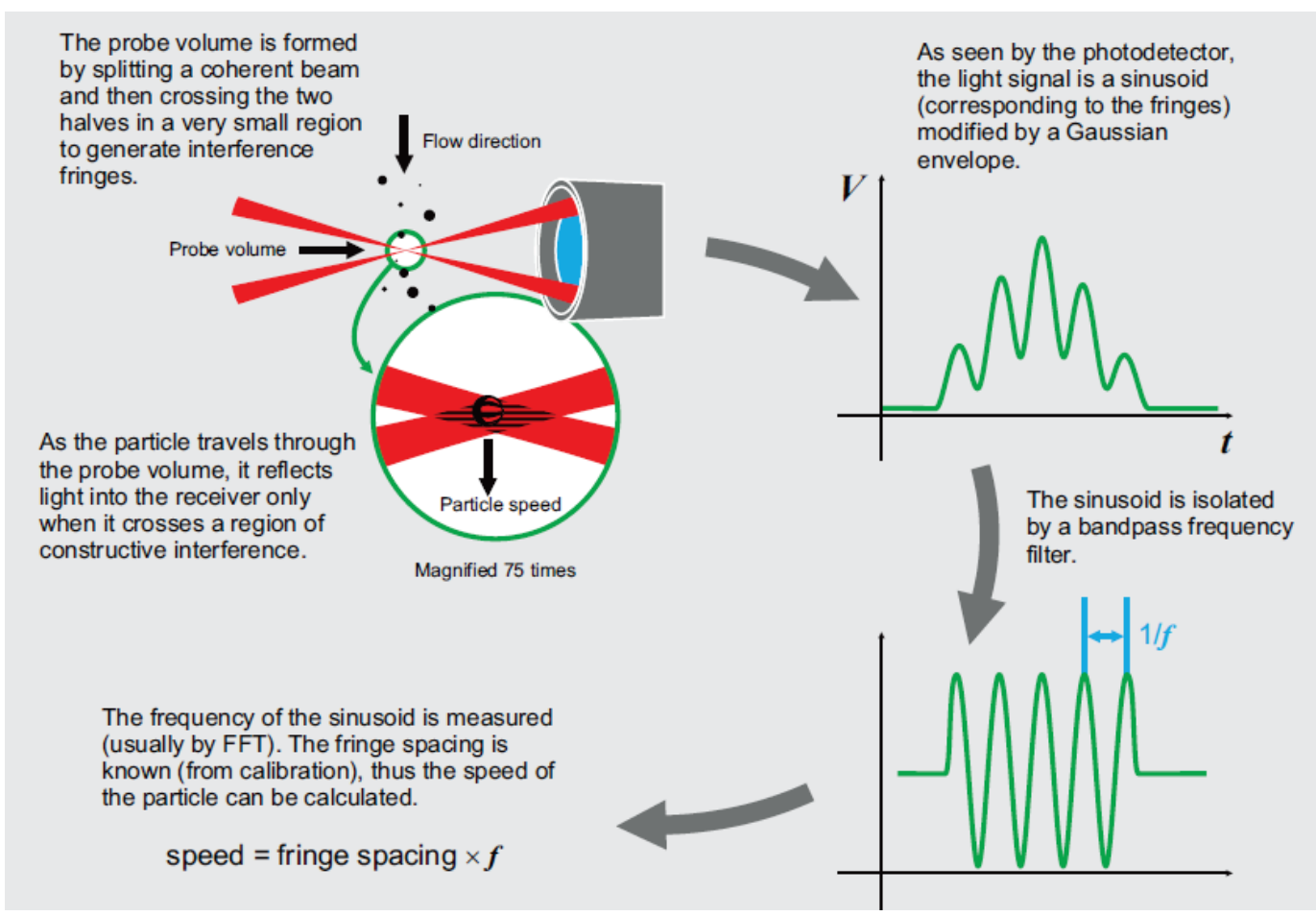

The miniLDV includes a frequency shifting component so that the fringes in the probe volume are moving at a constant velocity. The measurement then becomes one of the relative velocity differences between the particle in space and the fringes in the probe volume. Thus the probe can specify flow direction as well as measurement speed.

\section{Task 3-Demonstrate wave-current bottom shear-stress time histories representative of nature in SEAWOLF by manipulating flume and modulated flow conditions}

The bottom shear stress in combined current-wave conditions has been extensively studied and measured (Grant and Madsen 1979; Dibajnia and Watanabe 1992; Wikramanayake and Madsen 1994). These measurements have been used to develop reliable algorithms for bottom shear-stress time history under nonbreaking waves. The various algorithms produce fairly similar results and in general, vary as to the amount of parameterization used as input. For example, some methods require near-bottom velocity profile time series as input. This is reasonable for laboratory experiments but is not appropriate for natural systems where these data are not available. Available data generally include wave height and period as well as mean current velocity. Several studies have developed algorithms for nearbottom shear stress as a function of these more readily available 
parameters. As part of the objective to replicate naturally occurring wavecurrent conditions in SEAWOLF, these parameterized wave-current shearstress methods will be evaluated against calibrated hydrodynamic model output and used to program piston movement and head difference to produce a broad range of user-specified bottom shear-stress time histories in SEAWOLF.

The comprehensive database of shear-stress time histories collected with the optical sensors within SEAWOLF will be used to develop a means to program the computer-driven piston movement. A detailed parametric study, which includes all pertinent combinations of wave period, amplitude, unidirectional flow, and roughness regimes, is necessary to develop an understanding of how each parameter affects shear-stress time history in SEAWOLF. The resolution of the shear-stress time history database (i.e., number of tests performed) will determine the method used for filling in the data gaps for programming the SEAWOLF flume operation. High resolution in the database lends the opportunity for simple data fits to decipher the trends associated with variance in the individual parameters affecting shear-stress time history while a low resolution test matrix will produce a more uncertain extrapolative model. The previous near-wall PIV measurements provided high-quality results that revealed the nature of the SEAWOLF shear stress for a limited number of flow conditions. This existing database will be significantly augmented by a more detailed parametric study conducted using the miniLDV sensor, which will provide shear-stress data in a much more rapid and cost-effective manner.

\section{Task 4-Investigate uncertainty in shear-stress measurement techniques}

The wall shear stress was inferred from high-magnification PIV measurements of the velocity profile in the viscous sublayer using Equation 3. The impact of two primary causes of measurement error have been considered: (1) uncertainty in the viscosity, $\mu$, resulting from temperature variations during the approximate 4-hour duration of a given measurement run and (2) the confidence interval assigned to the leastsquares estimate of the slope of the velocity data, $d u / d y$, in the viscoussublayer region. 


\section{Results and Accomplishments}

\section{Task 1-Determine shear stress time histories and turbulent structure within the enclosed SEAWOLF channel with smooth and rough walls under modulated flow}

\section{Validation of PIV shear-stress measurements in a steady channel flow}

The validity of the PIV shear-stress measurements, obtained using the viscous-sublayer approach of Equations 3 and 6b, was investigated using data from steady turbulent flows established in the SEAWOLF test section. The steady turbulent channel flow provides an excellent test bed for the validation of the PIV shear-stress measurements because wall shear stress and velocity profiles are both well understood in steady channel flows. Wall shear-stress data were obtained from fits to both the linear and logarithmic laws of Equations $6 \mathrm{~b}$ and 10. The shear-stress results were additionally compared to the empirical correlation due to Blasius (Akhaven et al. 1991):

$$
\frac{2 \tau_{w}}{\rho U^{2}}=0.0791 R e_{d}^{-1 / 4}, \text { valid for } 4000<R e_{d}<10^{5}
$$

where $d$ is the hydraulic diameter of the channel and $U$ is the mass-mean or bulk velocity, $U=1 / 2 H \int_{0}^{2 H} u(y) d y$, with $H$ representing the channel half height and $u(y)$ the PIV-measured mean velocity profile.

Sample high-magnification PIV results from the steady channel flow at five different Reynolds numbers are shown in Figure 6. Mean velocity profiles were computed from ensemble and streamwise averaging of 500 statistically independent PIV realizations, as shown in the uppermost plot in Figure 8. The mean velocity data were normalized using inner-length scales (Equation 6), which were computed using shear-stress values obtained from fits of data in the logarithmic layer to Equation 13. At all Reynolds numbers (based on bulk velocity and hydraulic diameter), the velocity profiles display good collapse to a universal curve, with some scatter observed in the results closer to the wall, which is indicative of uncertainty in wall location and small differences in the shear-stress values computed from Equations 3 and 13. 
The resulting wall shear-stress data from viscous- and log-layer fits are provided as a function of Reynolds number in the lowermost plot in Figure 8. The experimental results are plotted alongside empirical values computed from Equation (22). Shear estimates from linear fits to the viscous layer are within $-4 \%$ to $-13 \%$ of both Equation (22) and results data obtained from fits to the log-layer expression, Equation (13). This was seen as reasonable level of accuracy for the measurements in the oscillating channel flow. Note that the excellent agreement (within a few percent) of the results from log-layer fitting and Equation (22) is likely fortuitous. The log-layer fits are subject to uncertainty in the exact value of the constant $B$ in Equation (13), and the Blasius correlation has additional uncertainty that is unknown to the authors at this time. There is no reason to believe that the viscous layer fits underpredict the true value of the shear stress, and it is the agreement of all three methods shown in Figure 8 to within $\sim 10 \%$ that is encouraging.

Phase-averaged (see below for a discussion of phase-averaging procedures) velocity profiles in the near-wall region for test-matrix Case 6 are presented in Figure 11. The profiles are presented in dimensional form to illustrate the high spatial resolution in the near-wall data and to provide a more intuitive link to the slope of the near-wall profile, shown as dark lines in Figure 12 and the wall shear-stress determined from Equation (3). For this test case, 51 mean velocity measurements within $1 \mathrm{~mm}$ of the testsection wall were obtained, from which at least 5 and as many as 11 points were available for linear fitting within the viscous sublayer.

The phase modulation of the wall shear-stress for all eight cases in the test matrix of Table 1 is presented in Figures 12 (10 sec wave cases) and 13 (5 sec waves). For each case, the wall shear stress (black circles) is plotted on the left-hand axis and the volume flow rate (white circles) on the right-hand axis. The shear-stress results were obtained from the slope of the near-wall velocity profiles, similar to those shown in Figure 11. A summary of the average shear stress along with the cycle maximum, minimum, and the amplitude [(maximum + minimum $) / 2]$ of the measured shear-stress waves is provided in Table 2. This table can be used as a starting point for the programming of the SEAWOLF facility for a desired shear-stress history. 
Table 1. Summary of experimental test matrix. Actual metered flow rates are shown, which may differ slightly than the target flow rates in some cases.

\begin{tabular}{|c|c|c|c|c|c|}
\hline Case \# & $\begin{array}{l}\text { Max. Flow } \\
\text { (GPM)* }\end{array}$ & $\begin{array}{l}\text { Min. Flow } \\
\text { (GPM) }\end{array}$ & $\begin{array}{l}\text { Mean Flow } \\
\text { (GPM) }\end{array}$ & Period (seconds) & Status \\
\hline 1 & 30 & -7 & 11.5 & 10 & $\begin{array}{l}\text { Complete wall shear- } \\
\text { stress and flowfield } \\
\text { data. Shaft encoder } \\
\text { used as a time base for } \\
\text { this case. Nonuniform } \Delta t \\
\text { between data points. }\end{array}$ \\
\hline 2 & 30 & -30 & 0 & 10 & $\begin{array}{l}\text { Completed high- } \\
\text { magnification PIV and } \\
\text { wall shear stress for } 20 \\
\text { of } 40 \text { phases. Low- } \\
\text { magnification images } \\
\text { have been acquired and } \\
\text { are currently being } \\
\text { analyzed }\end{array}$ \\
\hline 3 & 50 & -31 & 10 & 10 & $\begin{array}{l}\text { Complete wall shear- } \\
\text { stress and flowfield data }\end{array}$ \\
\hline 4 & 50 & -50 & 0 & 10 & $\begin{array}{l}\text { Complete wall shear- } \\
\text { stress and flowfield data }\end{array}$ \\
\hline 5 & 30 & -10 & 10 & 5 & $\begin{array}{l}\text { Complete wall shear- } \\
\text { stress and flowfield data }\end{array}$ \\
\hline 6 & 30 & -30 & 0 & 5 & $\begin{array}{l}\text { Complete wall shear- } \\
\text { stress and flowfield data }\end{array}$ \\
\hline 7 & 47 & -33 & 7 & 5 & $\begin{array}{l}\text { Complete wall shear- } \\
\text { stress and flowfield data }\end{array}$ \\
\hline 8 & 50 & -50 & 0 & 5 & $\begin{array}{l}\text { Complete wall shear- } \\
\text { stress and flowfield data }\end{array}$ \\
\hline
\end{tabular}

*GPM = gallons per minute

Table 2. Summary of wall shear-stress results.

\begin{tabular}{|l|c|c|c|c|c|c|c|c|}
\hline CASE \# & $\mathbf{1}$ & $\mathbf{2}$ & $\mathbf{3}$ & $\mathbf{4}$ & $\mathbf{5}$ & $\mathbf{6}$ & $\mathbf{7}$ & $\mathbf{8}$ \\
\hline Max. flow (GPM) & 30 & 30 & 50 & 50 & 30 & 30 & 47 & 50 \\
\hline Min. flow (GPM) & -7 & -30 & -31 & -50 & -10 & -30 & -33 & -50 \\
\hline Period (SEC) & 10 & 10 & 10 & 10 & 5 & 5 & 5 & 5 \\
\hline & & & & & & & & \\
\hline Mean shear (Pa) & 0.65 & -0.04 & 1.26 & -0.042 & 0.53 & 0.00 & 0.81 & -0.15 \\
\hline Max. shear (Pa) & 2.07 & 2.54 & 5.93 & 6.13 & 1.93 & 2.24 & 4.69 & 7.72 \\
\hline Min. shear (Pa) & -0.25 & -2.67 & 2.96 & -6.50 & -0.39 & -1.94 & -2.38 & -7.51 \\
\hline Amplitude (Pa) & 1.16 & 2.61 & 4.45 & 6.32 & 1.16 & 2.10 & 3.53 & 7.61 \\
\hline
\end{tabular}


For all cases, the form of the wall shear-stress history is significantly different than the volume flow rate. The phase lead of the shear stress relative to the flow is not particularly significant, which is evidence of significant turbulence during at least part of the cycle. As a check on consistency in the shear-stress data, note that the cases with zero mean flow $(2,4,6$, and 8$)$ display shear-stress waveforms that are nearly symmetric for the positive and negative flow portions of the cycle. Measured shear-stress maxima and minima for the zero-mean-flow cases are within $6 \%$ for three of the four zero-mean cases, with a maximum difference of $15 \%$ for Case 6 . This agreement in the peak values of the expected symmetric waveforms is an initial indicator of the precision of the shear-stress measurement.

The impact of the cyclic transition between laminar and turbulent flow is clearly observed in the shear-stress results, with a sharp increase in shear stress observed with transition to turbulence. This effect is especially apparent in the data for Cases 1 and 5, which display significant shearstress jumps during the later stages of the cycle acceleration. This shearstress jump was well documented with an observed flow transition observed in the PIV results.

For each plot in Figures 12 and 13, an additional curve labeled "quasisteady (turbulent)" has been added to the graph. This curve represents the computed value of the wall shear stress using the well-accepted, steady turbulent flow result from Blasius, given earlier in Equation (22), which can be rearranged to solve for the wall shear in terms of fluid properties and the measured mass-averaged velocity:

$$
\tau_{w}=0.0396 \rho U^{7 / 4}\left(\frac{v}{d}\right)^{1 / 4}
$$

where $v$ is the kinematic viscosity of water. The cycle-resolved, volumeaveraged velocity can be evaluated in SI units from the measured GPM data using

$$
U(\mathrm{~m} / \mathrm{s})=6.308 \times 10^{-5} \frac{\mathrm{GPM}}{A}
$$

where $A$ is the cross-sectional area of the SEAWOLF test section. The solid, black lines in Figures 12 and 13 were computed by applying the 
steady flow correlation in Equation (23) but using the unsteady GPM data to obtain the quasi-steady estimates in the figures. The quasi-steady results are in reasonable agreement in their predictions of average shear stress and shear-stress maximum and minimum for the eight cases studied here, so this formula may be a reasonable way to interpolate the shear results to other flow conditions. Equation (23) predicts the PIV-measured cycle-average shear stress to within $40 \%$ and the maximum or minimum shear to within $27 \%$, with some cases as good as $3 \%$ agreement. The correlation, in general, overpredicts the PIV-measured shear stress.

\section{Cyclic modulation of wall shear stress and turbulent properties in oscillating channel flow within the SEAWOLF flume}

A test matrix of eight scenarios (Table 1) with distinct wave current combinations was created for PIV testing. These scenarios adequately represent the range of operating conditions and are therefore adequate for CFD calibration. Low-magnification PIV studies of the cycle-resolved flow and turbulence in the SEAWOLF channel have been conducted for all eight cases in the test matrix of Table 1 . The consideration of just one of the eight cases in detail should be illustrative of the physics of flow in the flume. Detailed velocity and turbulence data for test matrix Case 6 will be discussed in this report. This case is in the oscillating flow regime, which is characterized by zero time-mean velocity (i.e., $U_{\min }=U_{\max }$ ). Test matrix cases with a nonzero time-mean flow are characterized as pulsatile, and the presence of the time-mean velocity component leads to one additional flowfield parameter. The low-frequency, large-amplitude test cases in the present test matrix yield pulsatile flow cases which appear to be at least qualitatively similar to the oscillating test cases investigated. Therefore, only a review of the physics of the oscillating case is provided here. A more detailed review, which will include physics relevant to pulsatile flow, will be presented in upcoming publications.

The reviews by Akhavan et al. (1991) and Salon et al. (2007) identify four regimes for wall-bounded oscillating Stokes flows, which are parameterized by the Stokes-thickness Reynolds number:

$$
\operatorname{Re}_{\delta}=U_{\mathrm{o}} \delta / v
$$

where $U_{\mathrm{o}}$ is the amplitude of the cross-sectional mean velocity oscillation and $\delta$ is the Stokes thickness: 


$$
\delta=\sqrt{2 v / \omega}
$$

$v$ is the kinematic viscosity and $\omega$ is the angular frequency of the velocity oscillation. Specifically, the four flow regimes are (1) laminar flow (L) for $R e_{\delta}<100$; (2) disturbed laminar flow (DL) at $100<R e_{\delta}<500$, where the velocity modulation displays only small perturbations about the laminar theory; (3) an intermittently turbulent flow (IT), which is initiated at $R e_{\delta} \sim$ 500-550; and (4) a full-cycle turbulence (T), which appears for higher $\operatorname{Re} \delta$ between 2600 and 3460 , based on the inspection of the shear-stress time histories in Figure 9 of Jensen et al. (1989).

All the PIV experiments were conducted in the IT regime, and those discussed here had Stokes-thickness Reynolds number of $\operatorname{Re}_{\delta}=1220$, 2033, and 2875 . The IT regime is characterized by rich physical behavior, with a cyclic relaminarization as a result of very strong favorable pressure gradient during the cycle acceleration phase, which is followed by a transition back to turbulent conditions during the decelerating or more weakly accelerating portions of the cycle. As $\operatorname{Re}_{\delta}$ increases, the fraction of the cycle for which the flow remains turbulent grows until full-cycle turbulence is eventually achieved.

A small number of quality experiments directed at turbulence structure in oscillating wall-bounded flows have been reported (Akhavan et al. 1991; Jensen et al. 1989; Hino et al. 1983). These efforts have elucidated the above-described cyclic turbulence-relaminarization-transition process and have provided quality turbulence data utilizing both laser-Doppler and thermal-anemometry techniques to yield pointwise results, but which do not provide any instantaneous picture of the $2 \mathrm{D}$ structure of the flow. To date, the authors are unaware of any reports of PIV measurements that do provide spatially correlated velocity data, and the present measurements fill this gap. The PIV results described here have also yielded extremely large vector ensembles, arguably resulting in better converged turbulence statistics than previously reported. The PIV implementation reported is additionally unique because a microscale PIV approach to a macroscale problem has been adopted in order to resolve the very-near-wall structure and because the dynamic velocity range of the PIV system is automatically adjusted throughout the cycle to compensate for the large, periodic swings in velocity magnitude. 


\section{Wall shear-stress behavior}

Phase-averaged velocity profiles in the near-wall region for $\operatorname{Re}_{\delta}=1220$ are shown in Figure 11. The profiles are presented in dimensional form to illustrate the high spatial resolution in the near-wall data and to demonstrate the quality of the fits to velocity data in the viscous sublayer, shown as dark lines in the figure. For this test case, 51 mean velocity measurements within $1 \mathrm{~mm}$ of the test-section wall were obtained, from which at least 5 and as many as 11 points were available for linear fitting within the viscous sublayer.

Figure 11. Representative fits to near-wall velocity profiles recorded in an oscillating boundary-layer flow at $R e_{\delta}=1220$. Data are shown in dimensional units and plotted on linear scales to illustrate the effectiveness of near-wall fitting.

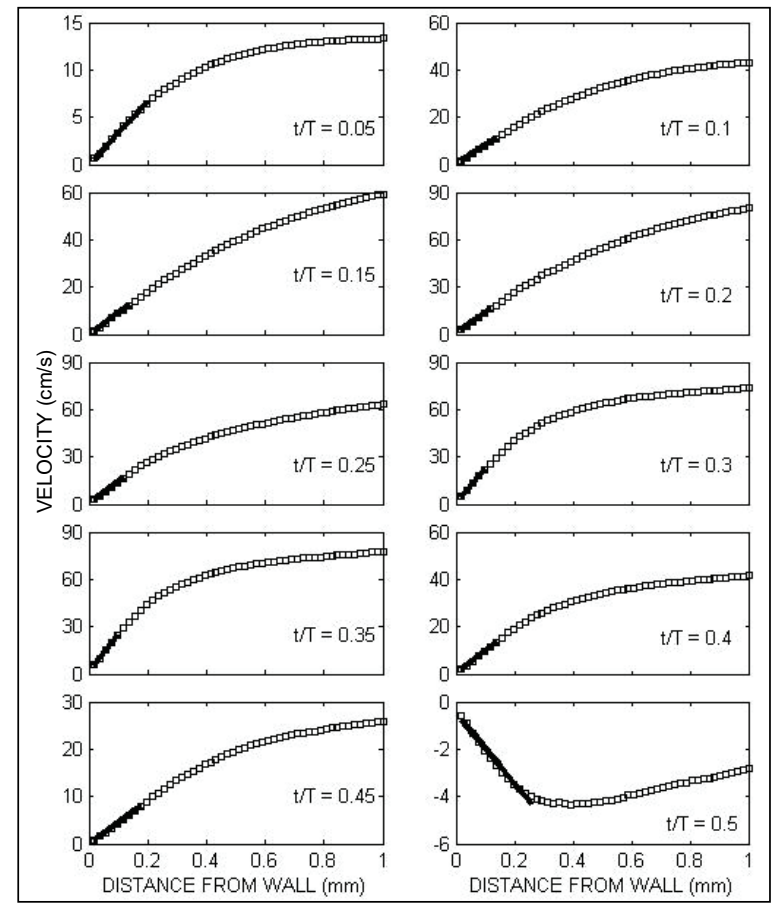


Figure 12. Phase-resolved shear-stress data for the test-matrix cases with $10 \mathrm{sec}$ wave period.
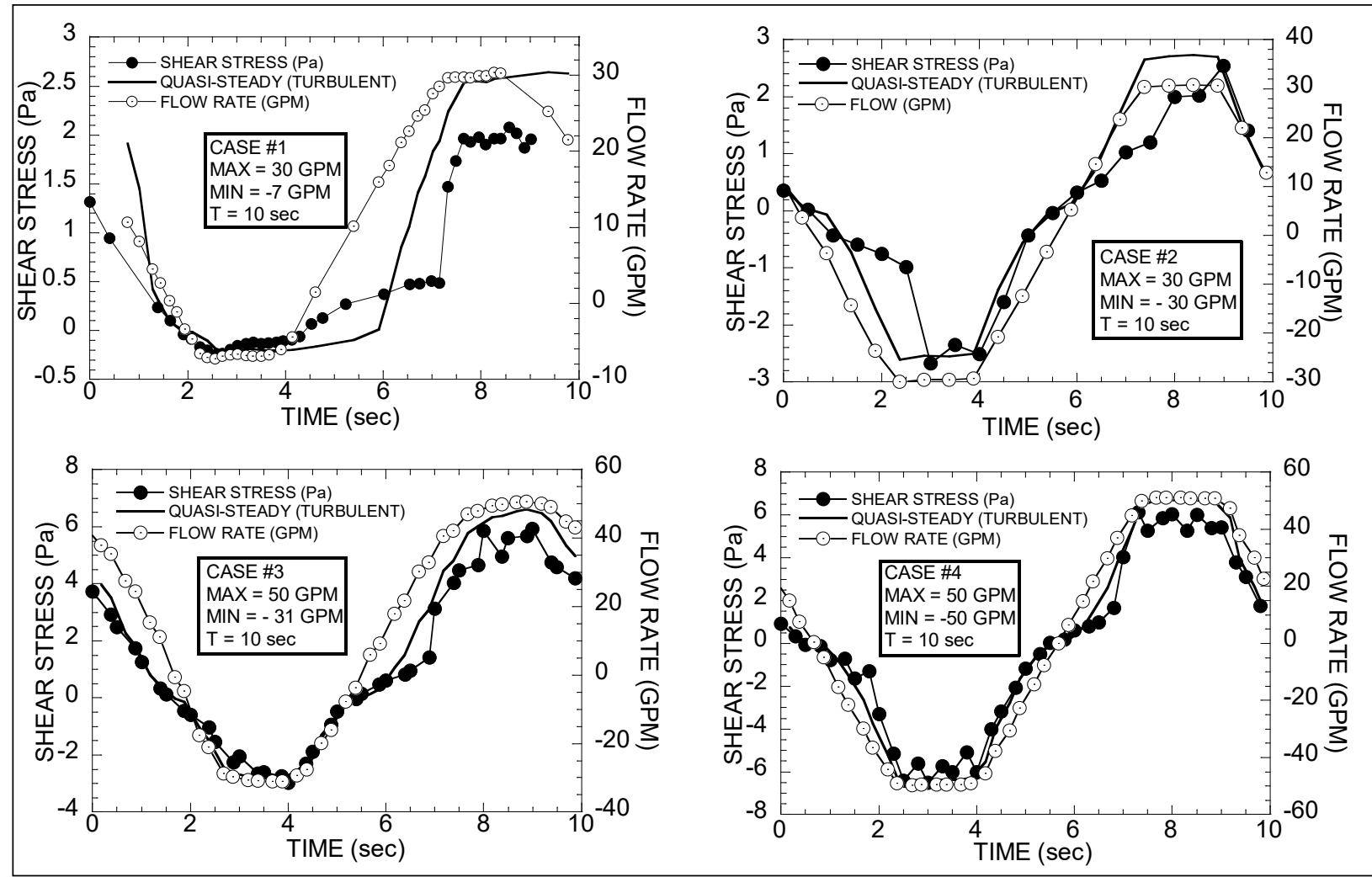

Figure 13. Phase-resolved shear-stress data for the $5 \mathrm{sec}$ wave period cases.
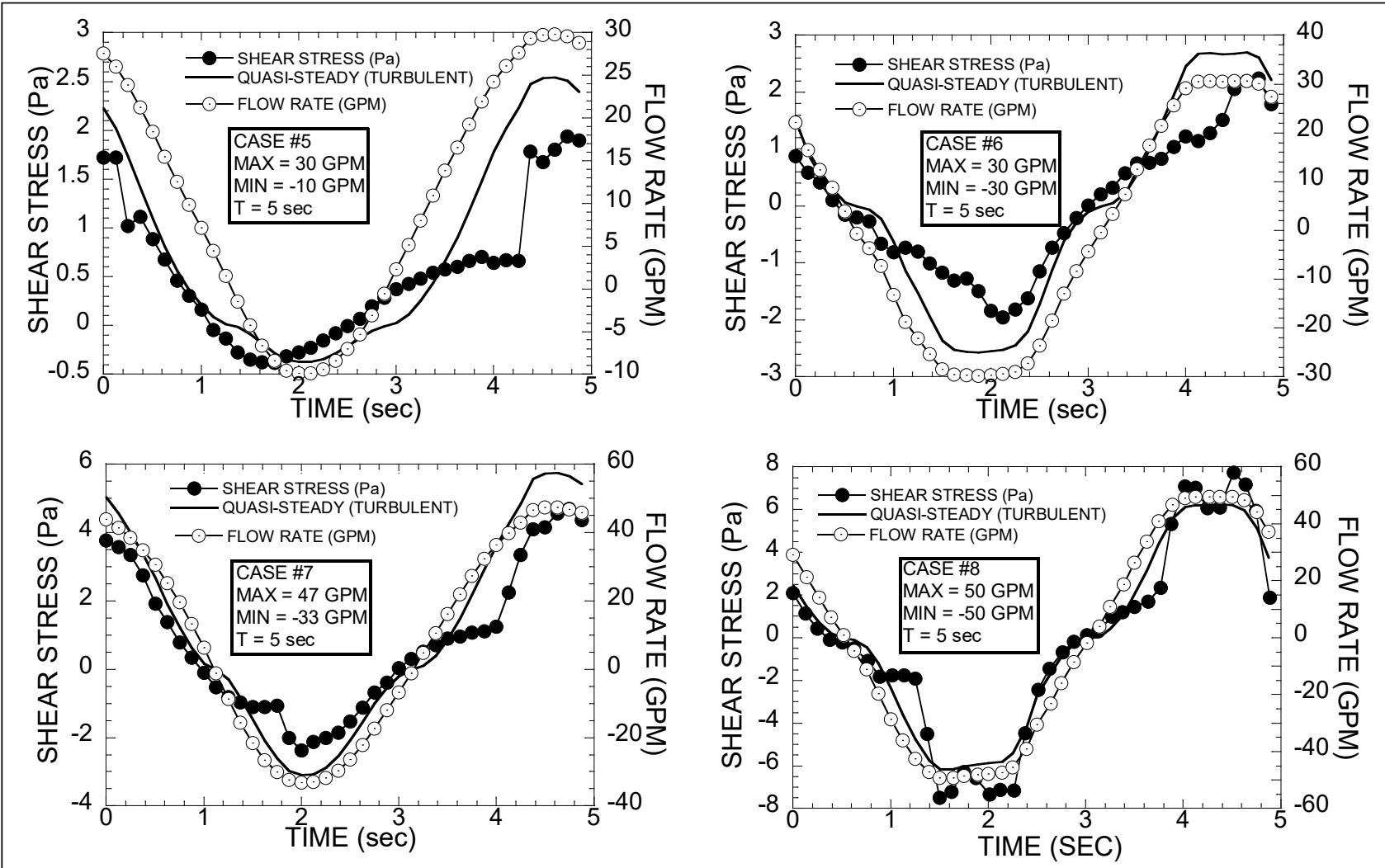
The phase-averaged modulation of the channel-averaged velocity is

$$
\langle U\rangle=\int_{0}^{2 H}\langle u\rangle(y) d y
$$

wall shear stress, turbulent kinetic energy, and production rate at $\operatorname{Re}_{\delta}=1220,2033$, and 2875 is displayed in Figure 14. The channelaveraged velocity is normalized by the amplitude of the average velocity waveform, $U_{0}$, while the wall shear-stress results are presented in terms of a phase-averaged friction coefficient, $C_{f}=2\left\langle\tau_{w}\right\rangle / \rho U_{0}{ }^{2}$. The time histories of the in-plane turbulent kinetic energy, $K$, have been averaged across the channel height and normalized according to

$$
\langle K\rangle=\frac{1}{2 H U_{o}^{2}} \int_{0}^{2 H}\left[\left\langle u^{\prime 2}\right\rangle+\left\langle v^{\prime 2}\right\rangle\right] d y
$$

and the channel-averaged, turbulence-production rate according to

$$
\langle P\rangle=\frac{\delta_{s}}{2 H U_{o}^{3}}=\int_{0}^{2 H}\left\langle u^{\prime} v^{\prime}\right\rangle \frac{\partial\langle u\rangle}{\partial y} d y
$$

where the Stokes thickness, $\delta$, has been selected as the outer length scale.

Figure 14. Phase modulation of the bulk velocity, wall shear stress, and channel-averaged turbulence.

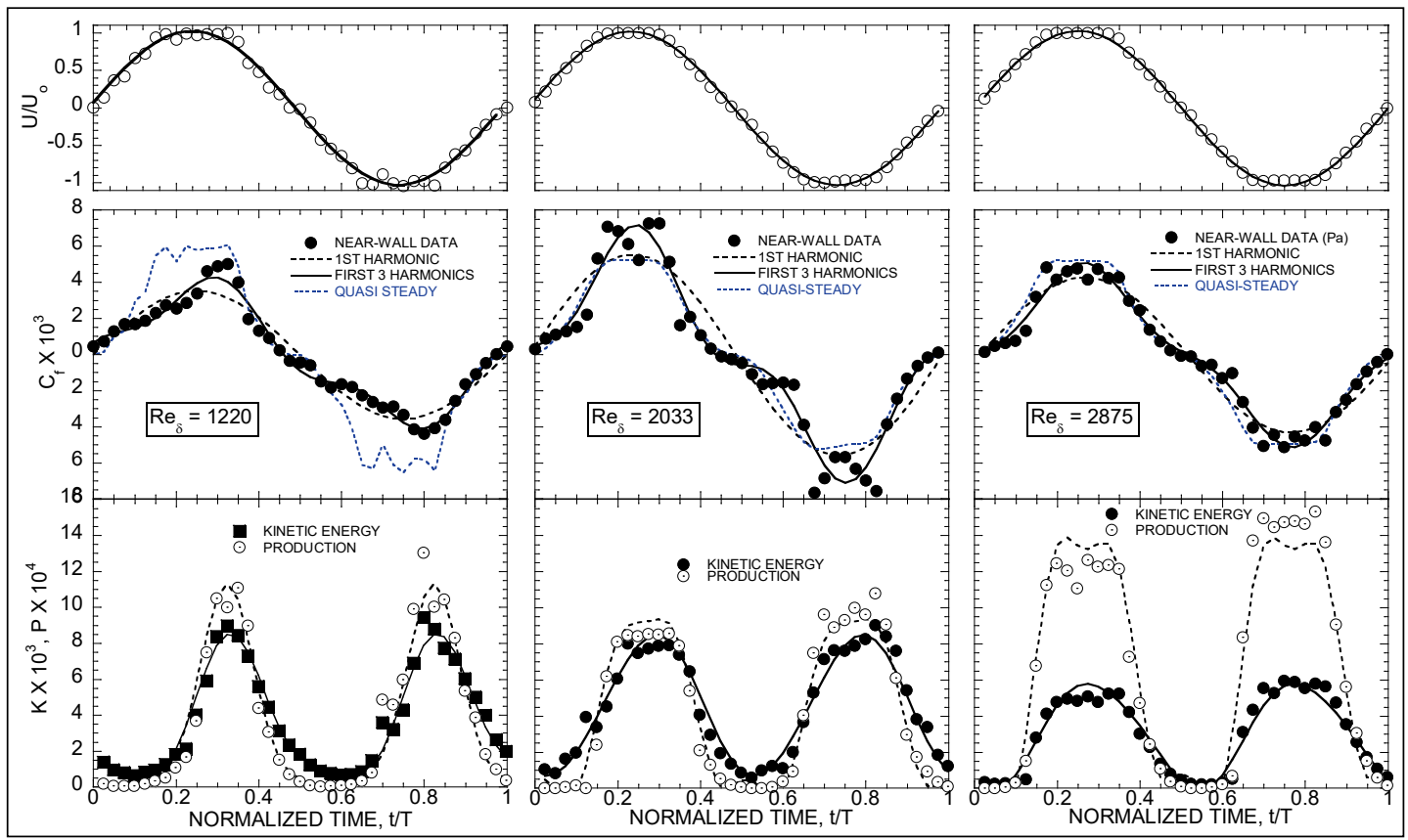


For all cases, the form of the wall shear-stress history is significantly different than the nearly sinusoidal modulation of the bulk flow, as three terms of a Fourier-series representation of the wall shear-stress data are required to capture the main features of the shear-stress oscillation. At all three Reynolds numbers, the first harmonic of the measured shear-stress data reveals no significant phase lead of the wall shear relative to the bulk velocity. As a check on consistency in the shear-stress data, note that the shear-stress waveforms are nearly symmetric for the positive and negative flow portions of the cycle, with measured shear-stress maxima and minima within $15 \%$.

The impact of a cyclic transition between laminar and turbulent flow is clearly observed in the modulation of $\langle K\rangle$ and $\langle P\rangle$, as well as in the wallfriction results. At each Reynolds number, the positive-velocity half cycle begins with $\langle P>$ at essentially zero value and residual, decaying turbulent kinetic energy from the previous half cycle. The flow is relaminarized in the presence of a strong, favorable pressure gradient during the acceleration phase, with an explosive transition to turbulence observed in the mid-tolate acceleration, dependent on the value of $\operatorname{Re}_{\delta}$. The transition is marked by a rise in $\langle P>$ from near-zero values, and this rise is accompanied by a sudden change in the slope of the friction-coefficient-vs.-time curve. This jump is particularly apparent at $R e_{\delta}=2033$ and 2875 . Note that production of turbulence ceases for some fraction of the cycle, even at $R_{\delta}$ as high as 2875. If a laminar state is defined as one where $\langle P\rangle$ is at near-zero value, then it is evident that the laminar portion of the cycle is $40 \%-50 \%$ less duration at $\operatorname{Re}_{\delta}=2875$ than at $\operatorname{Re}_{\delta}=1220$, based on inspection of the phaseaveraged histories of $\langle P>$ in Figure 14 .

For comparison, an additional curve labeled "quasi-steady," and shown in blue, has been added to each of the $C_{f}$ data plots in Figure 14. These curves represent the computed value of $C_{f}$ from Equation (14) when using the phase-averaged velocity to compute the hydraulic-diameter-based Reynolds number, $R e_{d}$. The quasi-steady results are in reasonable agreement in their predictions of average shear stress and shear-stress extrema. Equation (14) predicts the PIV-measured cycle-average shear stress to within $40 \%$, and the maximum or minimum shear to within $27 \%$, with some cases as good as $3 \%$ agreement. This level of agreement suggests that the quasi-steady approach may provide reasonable engineering accuracy. 


\section{Visualizing the bulk flow in the intermittently turbulent flow (IT) regime (introduction to flow structure)}

The case at $R e_{\delta}=1220$ is examined here in more detail to illustrate the instantaneous and phase-averaged structure of the oscillating channel flow in the IT regime. The cyclic relaminarization and transition process is well visualized using instantaneous PIV recordings at low magnification, as shown in Figures 15 and 16, for the acceleration and deceleration phases of the half cycle, respectively. The vector fields are shown across the full depth of the channel, and the instantaneous value of the globally averaged streamwise velocity for each realization has been subtracted from the data to better reveal the structure of any turbulence. Vectors are displayed using an arbitrary false-color scale as an indicator of the velocity magnitude; the color scale indicates the difference between the lab-referenced and convective velocities and is red for slow-moving fluid and blue for fastermoving fluid in the lab frame of reference. At $t / T=0.05$, the velocity field has just experienced a zero crossing, and organized, large-scale structure is observed across nearly the full height of the channel. Production of turbulence is negligible at this time, and the turbulent kinetic energy is decaying rapidly. Relaminarization then abruptly occurs, with the vector fields at $t / T=0.10$ and 0.15 displaying a flat, plug-flow velocity profile in the core that is devoid of any organized large-scale structure. The young laminar boundary layers on the channel walls (observed as red-yellow in Figure 15) thicken even as the flow is accelerated. By $t / T=0.25$, an instability is observed near the channel ceiling and in the core flow; production and turbulent kinetic energy begin to increase, and flow reaches the end of the acceleration phase of the half cycle.

Instantaneous vector fields for the decelerating portion of the half cycle are shown in Figure 16. By $t / T=0.35$, when the bulk flow just starts to decelerate, an explosive transition to turbulence has occurred; large-scale structure appears in the core of the channel, and turbulent kinetic energy and production are at their cyclic maxima. Turbulence persists throughout this period of strong adverse pressure gradient, through $t / T=0.40$ and 0.45 , while the rate of production and kinetic energy decline. By $t / T=0.5$, production of turbulence has ceased, and only residual, decaying, but apparently large-scale structures remain. The bulk flow then changes direction again, and this process repeats. 
Figure 15. Representative instantaneous velocity vector fields obtained during the acceleration phase of the half cycle at

$$
R e_{\delta}=1220 \text {. }
$$
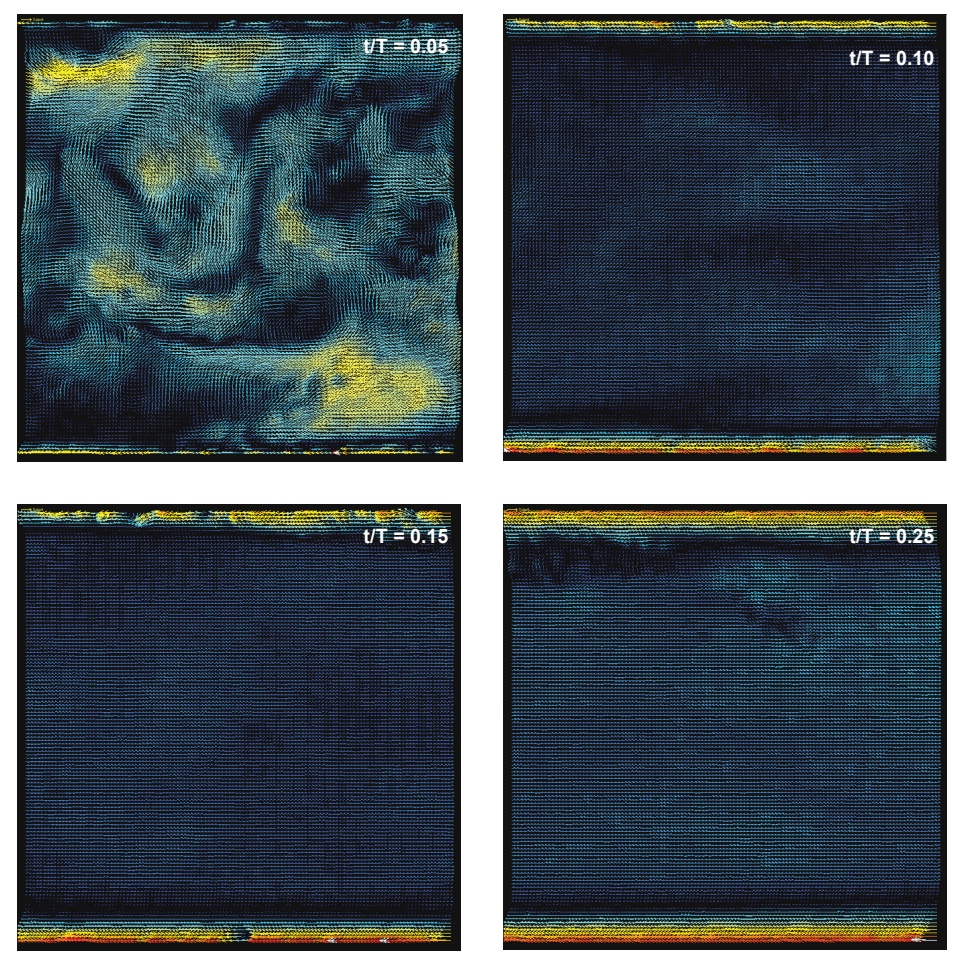

Figure 16. Representative instantaneous velocity vector fields obtained during the deceleration phase of the half cycle at

$\operatorname{Re}_{\delta}=1220$.
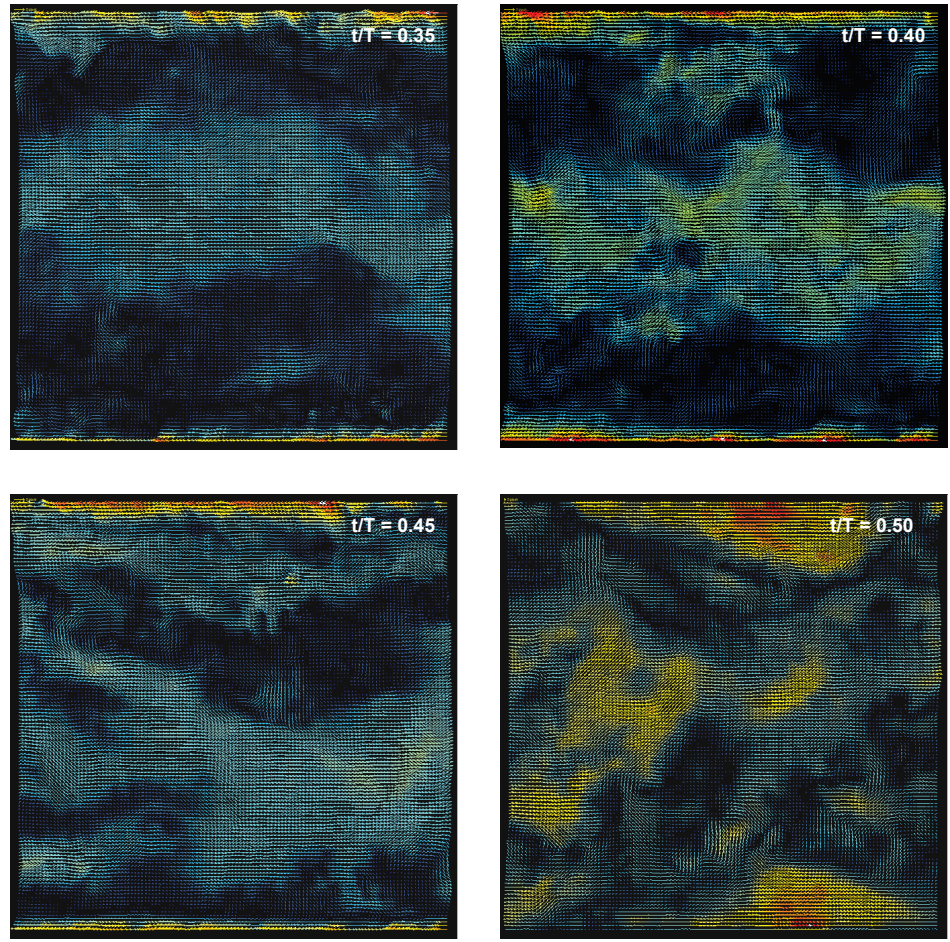
Profiles of the phase-averaged streamwise velocity in the near-wall region are presented in terms of the inner variables in Figure 17. The profiles are shown for $\operatorname{Re}_{\delta}=1220$, and these data have been scaled using the phaseresolved values of the wall shear stress shown in Figure 14 for computation of a cycle-varying friction velocity and viscous length scale. In this manner, a comparison to the law of the wall for steady, equilibrium boundary layers can be readily made. The outstanding spatial resolution of the highmagnification PIV results readily allows an observation of the structure of the boundary layer from the logarithmic region to well inside the viscous sublayer. In each profile, the viscous sublayer and logarithmic laws (using $\kappa=0.41$ and $B=5$ ) for steady boundary layers are plotted for reference. It is evident that the velocity departs significantly from the logarithmic law for a large portion of the cycle so that fits of log-layer data cannot be used to estimate the wall shear stress, and direct probing of the viscous sublayer is required. The half cycle begins at $t / T=0.05$ with an inflection point in the profile, and the velocity data lie well below the log law. By $t / T=0.1$, the flow has relaminarized, and logarithmic behavior is not observed until $t / T=0.3$, where the constant, $B$, in a log-law fit to the data is $\sim 6.5$ for $\kappa=$ o.41. This logarithmic behavior persists through the remainder of the half cycle, with $B$ gradually decaying to a late-cycle value near 5.0 that is typically observed in steady boundary layers, albeit with decreased quality of the logarithmic fit.

Wall-normal profiles of the turbulent stresses at $\operatorname{Re}_{\delta}=1220$ in the plane of the low-magnification PIV measurements are presented in Figure 18. The low-magnification PIV results reveal the cyclic evolution of the turbulence quantities across the channel half height. These results have been normalized using the amplitude of the velocity oscillation, $U_{\mathrm{o}}$, and the Stokes thickness as velocity and length scales, so that the data are more readily compared to results from the literature (Jensen et al. 1989; Salon et al. 2007). The Reynolds normal stresses, $\left\langle u^{\prime 2}\right\rangle$ and $\left\langle v^{\prime 2}\right\rangle$, have been reported in terms of their square roots (e.g., $u_{r m s}=\sqrt{\left\langle u^{\prime 2}\right\rangle}$ ) so that variations in these quantities are also more readily evident. The Stokes thickness is further indicated by the dashed lines on each of the plots in Figure 18. 
Figure 17. Near-wall mean velocity profiles at $\operatorname{Re}_{\delta}=1220$ displayed in inner units. The viscous scales vary throughout the cycle and have been computed using phase-averaged values of the wall shear stress.

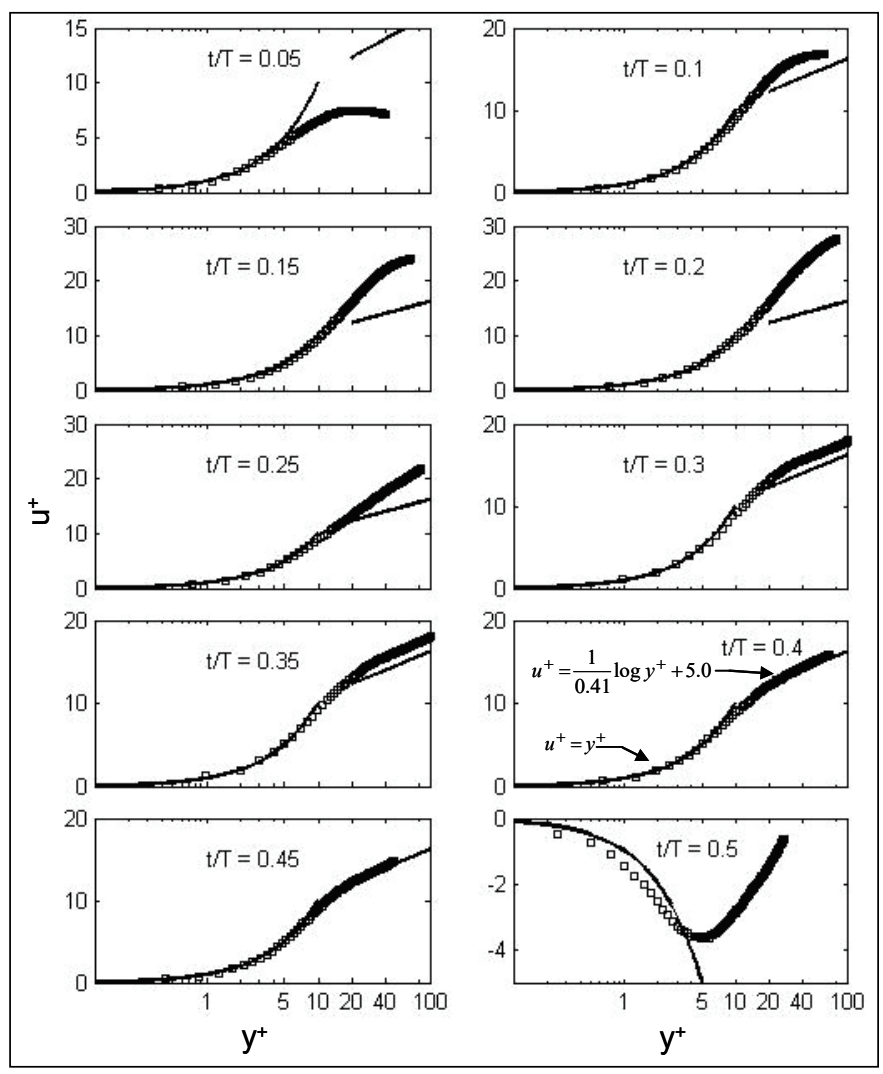

The profile of the streamwise turbulence intensity, $u_{r m s}^{\prime}$, is flat and near zero at the beginning of the half cycle. From there, measurable turbulent fluctuations begin very near the wall and within the Stokes layer at $t / T=$ 0.1; the growth of the streamwise fluctuations emerges from the wall and penetrates to the core of the channel flow by $t / T=0.3-0.35$, near the crest of the bulk velocity waveform, after which the strength of the streamwise fluctuations decay, and the profiles flatten in shape until a flat but measurable streamwise fluctuation remains just after the zero-velocity crossing near $t / T=0.5$. The maximum amplitude of the streamwise fluctuations remains in the Stokes layer throughout the cycle, with its maximum value of $u_{\mathrm{rms}} / U_{o} \sim 0.17$ at $t / T=0.25$, which occurs as the flow in the core of the channel is just beginning to transition from laminar to turbulent conditions (Figures 15-16), with the corresponding jump in the wall shear stress, as seen in Figure 15. The streamwise fluctuations remain near this maximum value until the deceleration phase of the cycle begins during which $u_{r m s}$ decays. 
Figure 18. Phase-averaged modulation of the profiles, in outer units, of the turbulence quantities across the channel half height at $R e_{\delta}=1220$, as acquired using low-magnification PIV. Streamwise turbulence intensity, $u_{r m s} / U_{0}$; vertical turbulence intensity, $v_{r m s} / U_{0}$; Reynolds shear stress, $\left\langle u^{\prime} v^{\prime}\right\rangle / U_{0}{ }^{2}$; and rate of turbulence production, $\delta_{s} / U_{0}^{3}\left\langle u^{\prime} v\right\rangle \partial\langle u\rangle / \partial y$. Distance from the wall is normalized by the Stokes thickness, $\delta_{s}$.

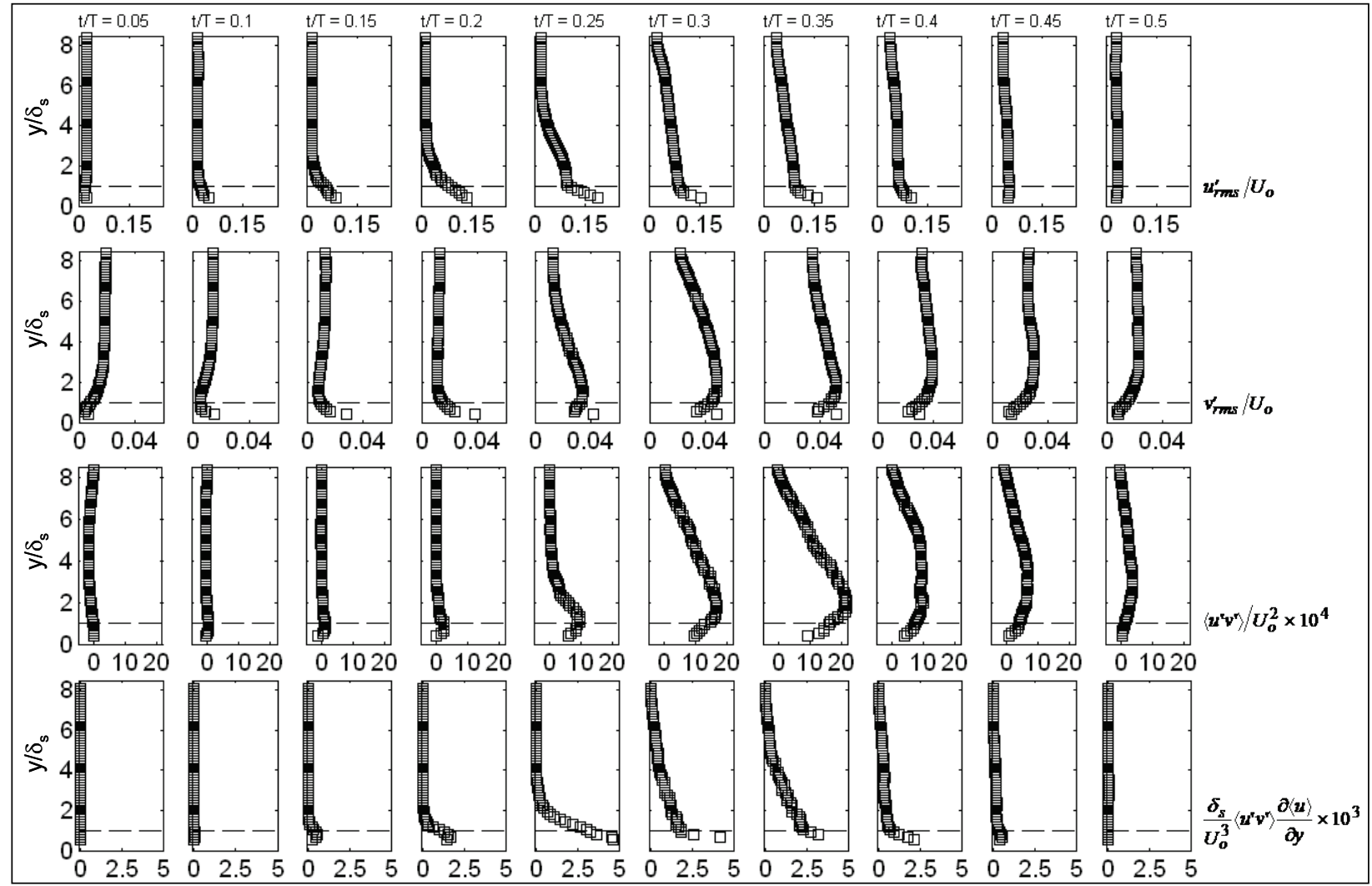

The behavior of the streamwise fluctuation profiles just described is similar to the results of Salon et al. (2007) at $R e_{\delta}=1790$ and the LDV measurements of Jensen et al. (1989) at $R e_{\delta}=1000$ and 1790. Both of these earlier studies report the same near-wall generation of fluctuations and subsequent wave-like penetration of these fluctuations into the core flow, which reaches its penetration maximum early in the deceleration

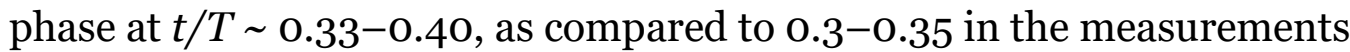
at $R e_{\delta}=1220$. Jensen et al. (1989) report maximum streamwise fluctuations of $u_{\mathrm{rms}} / U_{o} \sim 0.1$ to 0.12 at $R e_{\delta}=1000$ and 1790 while Salon et al. (2007) report maximum values of $u_{r m s} / U_{o}$ near 0.15 , which can be compared to a value near 0.17 in the PIV data.

Vertical velocity-fluctuation profiles in Figure 18 reveal that peak wallnormal fluctuations are approximately four to five times lower than the streamwise fluctuations observed in this flow. Peak vertical fluctuations are in the vicinity of $v_{r m s} / U_{o} \sim 0.05$, compared to values of 0.04-0.045 measured by Jensen et al. (1989) at $\operatorname{Re}_{\delta}=1000$ and 1790, and computed 
by Salon et al. (2007) at $R e_{\delta}=1790$. At $t / T=0.05$, the measured vertical fluctuations display a monotonically increasing profile, with values in the core that are comparable to the streamwise fluctuations at this time. The vertical-fluctuation profiles then decay in the channel core as the bulk flow is relaminarized, with a second inflection point observed at a height corresponding to the Stokes thickness discernable at $t / T=0.10$ and 0.15 . The vertical fluctuations then proceed outward toward the channel core in the same wave-like manner displayed by $u_{r m s}$, decaying to similarly flat profiles with no near-wall maxima by the end of the forcing cycle.

Profiles of the Reynolds shear stress, and rate of turbulence production reveal that there is essentially zero production early in the half cycle, production begins very close to the wall at $t / T \sim 0.15$, and the turbulent shear-stress and production emerge from the near-wall region toward the core flow in the same wave-like manner discussed above. Production of turbulence is confined to within $5 \delta$ s from the wall, with the maximum local value of production occurring within the Stokes layer at $t / T=0.25$ and the peak integrated production at $t / T=0.35$.

\section{Turbulence structure in oscillating channel flow}

The PIV data were phase locked with respect to the piston motion. Data at 40 evenly spaced phase angles were acquired, with 200 velocity realizations acquired at each phase angle (80oo total velocity field realizations). For the test condition to be discussed here, the period of the piston forcing was 5 $\mathrm{sec}$, with a $U_{\mathrm{o}} \pm 100 \mathrm{~cm} / \mathrm{sec}$ amplitude velocity wave and zero time-mean flow through the channel. These conditions yield a Stokes thickness of $\delta=$ $1.23 \mathrm{~mm}$ (from Equation 26). The Reynolds number, from Equation 19, was 2033, which is in the fully turbulent regime identified by Jensen et al. (1989). Bulk-velocity, wall shear stress, and turbulence production/kinetic energy waveforms for the test case are provided in Figure 19. Under these conditions, the shear stress varied over a \pm 7.5 Pa range, such that the minimum size of the viscous length scale was $11 \mu \mathrm{m}$, occurring during periods of peak wall shear stress. The spatial resolution of the PIV measurements in the streamwise/wall-normal plane was $160 \mu \mathrm{m}$, or 14.5 minimum viscous wall units. This represents a worst-case spatial resolution, as the PIV vector spacing in wall units correspondingly improves for offpeak shear-stress conditions, where the phase-resolved value of the viscous length scale is larger. 
Figure 19. Phase-averaged modulation of bulk velocity (top); wall skin friction (middle); in-plane turbulent kinetic energy and turbulence production integrated across the channel height (bottom).

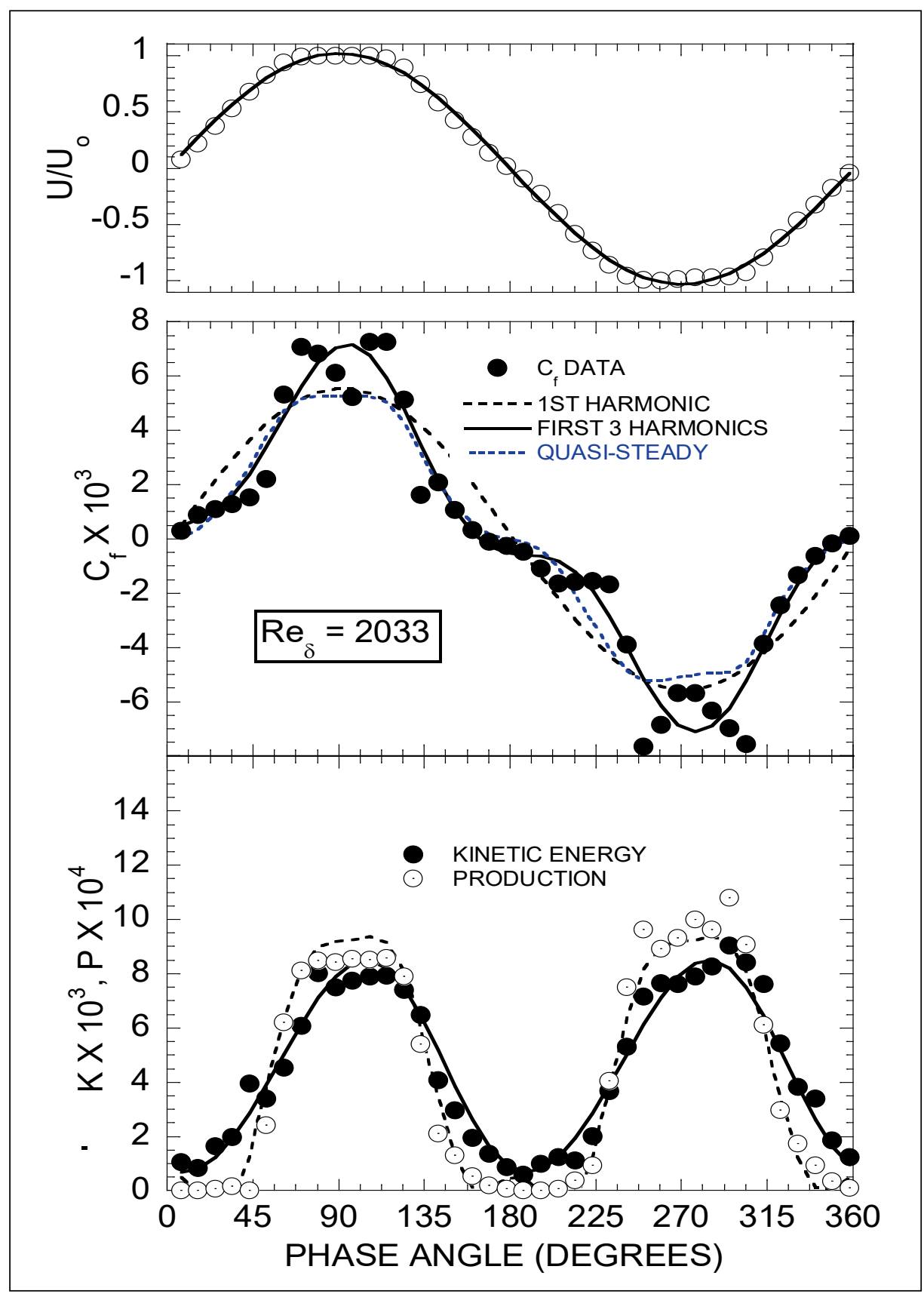

Instantaneous flowfield structure

Representative instantaneous velocity vector realizations are shown in Figures 20 and 21 for the accelerating and decelerating portions of the first half cycle, respectively. The vector fields are plotted for the lower half of the channel over a streamwise distance, $x / H$ of 1.8 , where $H$ is the channel half height. A streamwise convective velocity, $U_{c}$, of $85 \%$ of the phase-locked 
mean at the channel centerline has been subtracted from the vector fields to reveal the structure of turbulence in a reference frame that is nominally moving with the near-wall flow. Any organized vortical structure that is traveling at $U_{c}$ will appear as the closed, circular vector patterns that are associated with vortex motion. The vectors are also plotted on top of grayscale contours of swirling strength, $\lambda_{c i}$, which is an accepted frameindependent kinematic identifier of vortex motion (Adrian et al. 2000a) that marks the location of vortices traveling at speeds other than $U_{c}$.

Instantaneous turbulence structure during the acceleration phase $\left(0^{\circ}\right.$ to $90^{\circ}$ ) of the half cycle is shown in Figure 20 . At $8^{\circ}$ and $17^{\circ}$, the bulk velocity is near zero, and reversals of the bulk flow direction and sign of the channel pressure gradient have just occurred. At this time, production of turbulence has ceased (Figure 15), and turbulence from the decelerating portion of the previous half cycle persists and is dominated by large-scale vertical up- and downwash motions. Pockets of swirling strength are observed throughout the channel. By $35^{\circ}$ and $53^{\circ}$, the impact of strong acceleration is felt throughout the flow. Production remains essentially zero at $35^{\circ}$, and weak swirling strength events are generally limited to a near-wall region for $y / H$ $<0.2$; the flow has essentially relaminarized. At $53^{\circ}$, production has begun, with some vertical ejection of swirling motion from the wall observed in some fraction of the realizations, such as the one shown in Figure 20. The acceleration phase ends near the peak of the velocity wave, where the structure is typical of that seen at $71^{\circ}$; production is near cycle-peak values, an organized procession of strong vortex cores reminiscent of hairpin vortex signatures (Adrian et al. 200ob) appears (more evidence below), and a logarithmic phase-mean velocity profile (Kearney et al. 2008) is established.

Instantaneous vector and swirl fields during the decelerating portion of the half cycle are displayed in Figure 21, where the evolution of the turbulence in the presence of a strong deceleration is visualized. At $89^{\circ}$ and $107^{\circ}$, the flow is in the same quasi-equilibrium state as at $71^{\circ}$ above, with a succession of hairpin vortex cores travelling near $U_{c}$ clearly visualized at $107^{\circ}$. By $125^{\circ}$, a weak deceleration has initiated. By $161^{\circ}$, production of turbulence is still nonzero, and organized vortical structures are still observed. The half cycle ends near $179^{\circ}$, where the mean velocity profile reveals that the flow in the near-wall region has already changed direction, turbulence production has reverted to zero levels, and very-large-scale turbulence, similar to that observed near the start of the half cycle at $8^{\circ}$, reappears. 
Figure 20. Instantaneous velocity vector measurements during the acceleration portion of the half cycle.

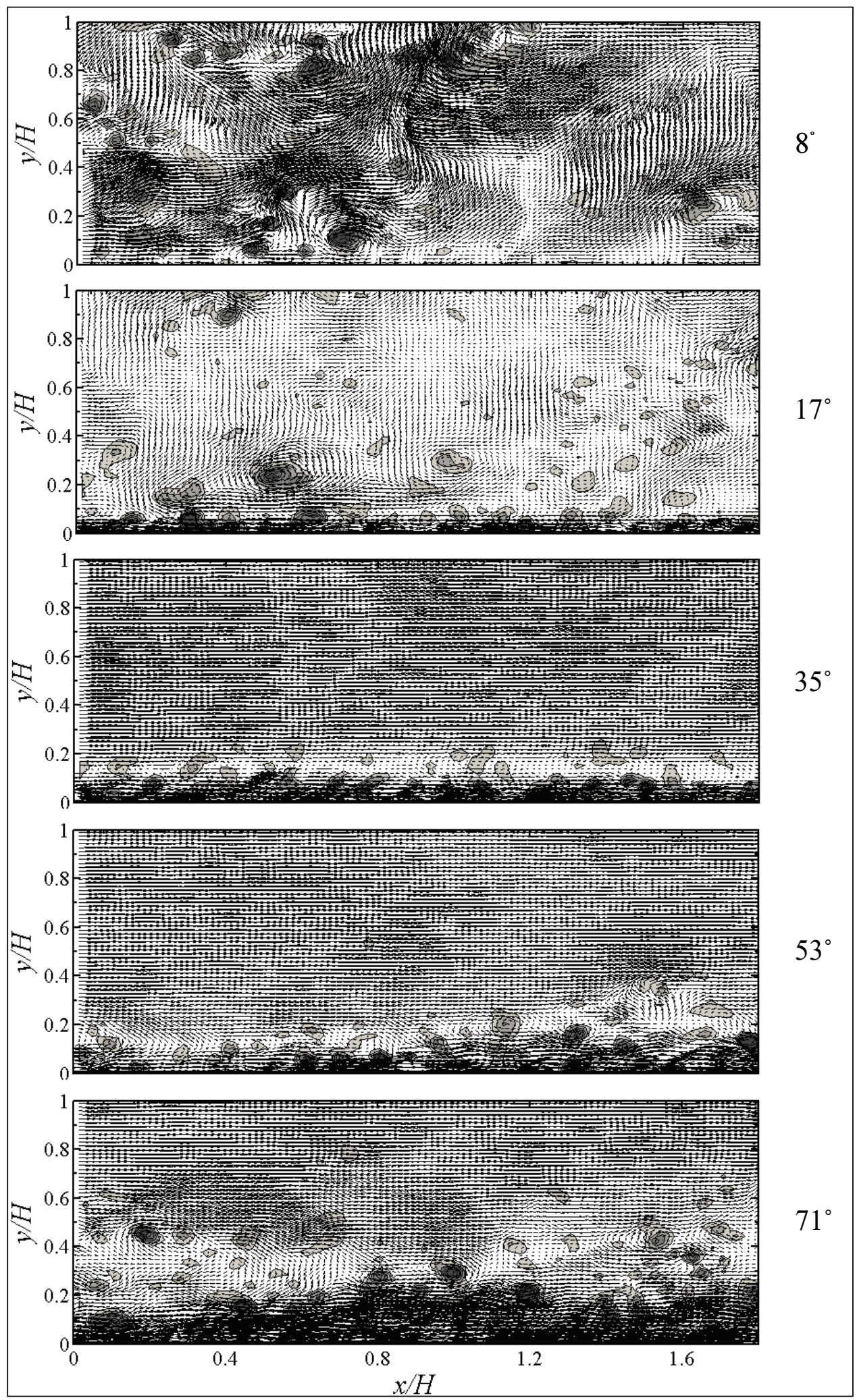


Figure 21. Instantaneous velocity vector measurements during the deceleration portion of the half cycle.
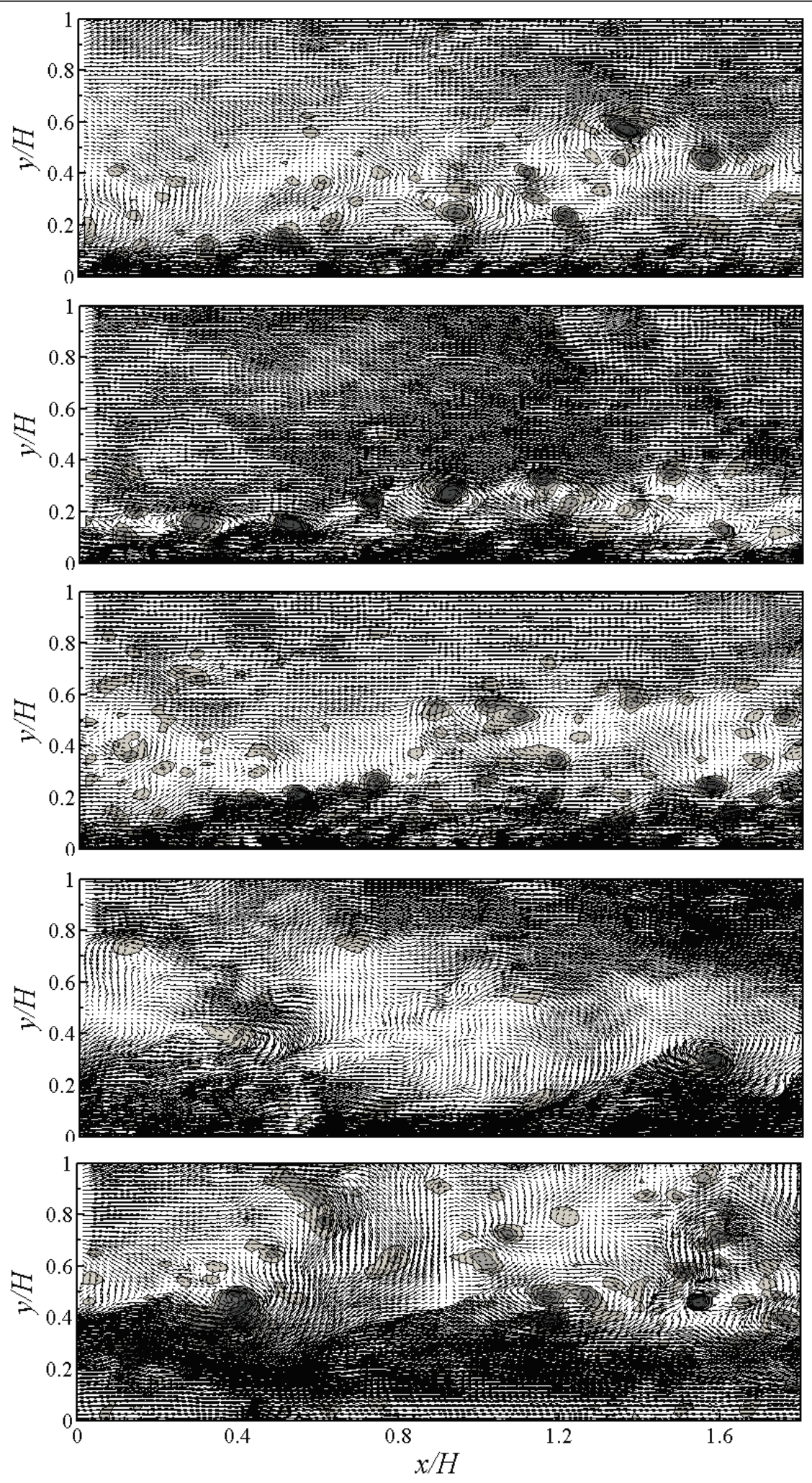


\section{Two-point spatial correlations}

The time evolution of the two-point correlation fields is observed in

Figures 22 and 23, where contour plots of $R_{u u}$ and $R_{v v}$ are shown. Note the different scales on the horizontal axes in Figures 22 and 23, which were used because the streamwise extent of the $R_{u u}$ correlation was much larger than for $R v v$. For quick visualization of the mean size and orientation of organized turbulent structure, contours for $R$ values higher than 0.5 are filled with grayscale colors, with lower-valued contours unfilled. The small ensemble size (200 PIV realizations) used to compute the correlations provides only marginal convergence, but this is still sufficient to visualize the streamwise evolution of these quantities. Larger PIV image ensembles, while desirable, were not practical as a result of the unsteady, phase-locked nature of the experiments, which were costly, taking 6-8 hours each for setup and acquisition of 200-250 forcing cycles.

Figure 22. Two-point spatial correlation of the streamwise velocity fluctuations at 10 different phase angles over the first half cycle. The event location, $y_{\text {ref, }}$ is located at $y / H=0.137$.

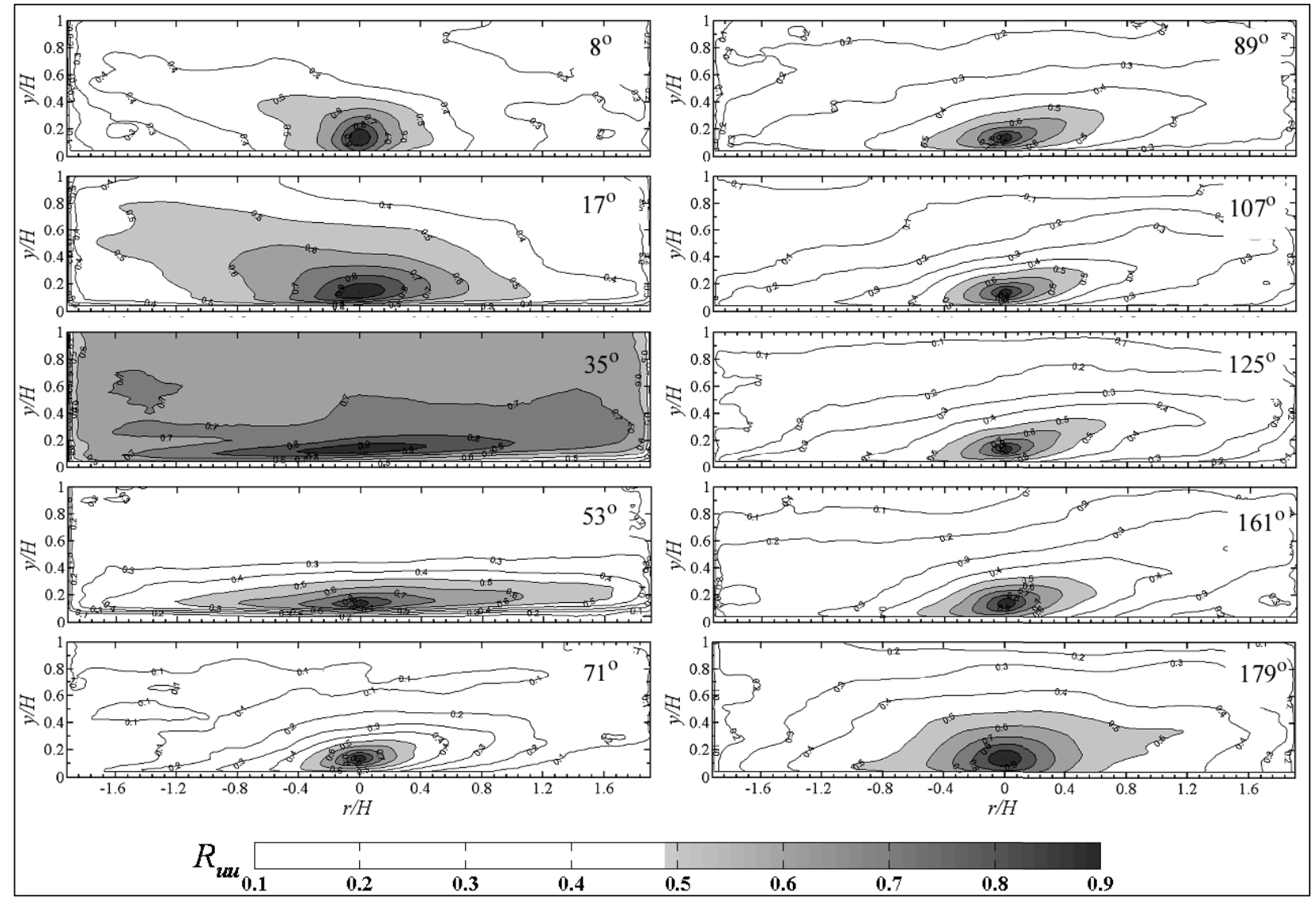


Figure 23. Two-point spatial correlation of the wall-normal velocity fluctuations at 10 different phase angles over the first half cycle. The event location, $y_{r e f}$, is located at $y / H=0.137$.

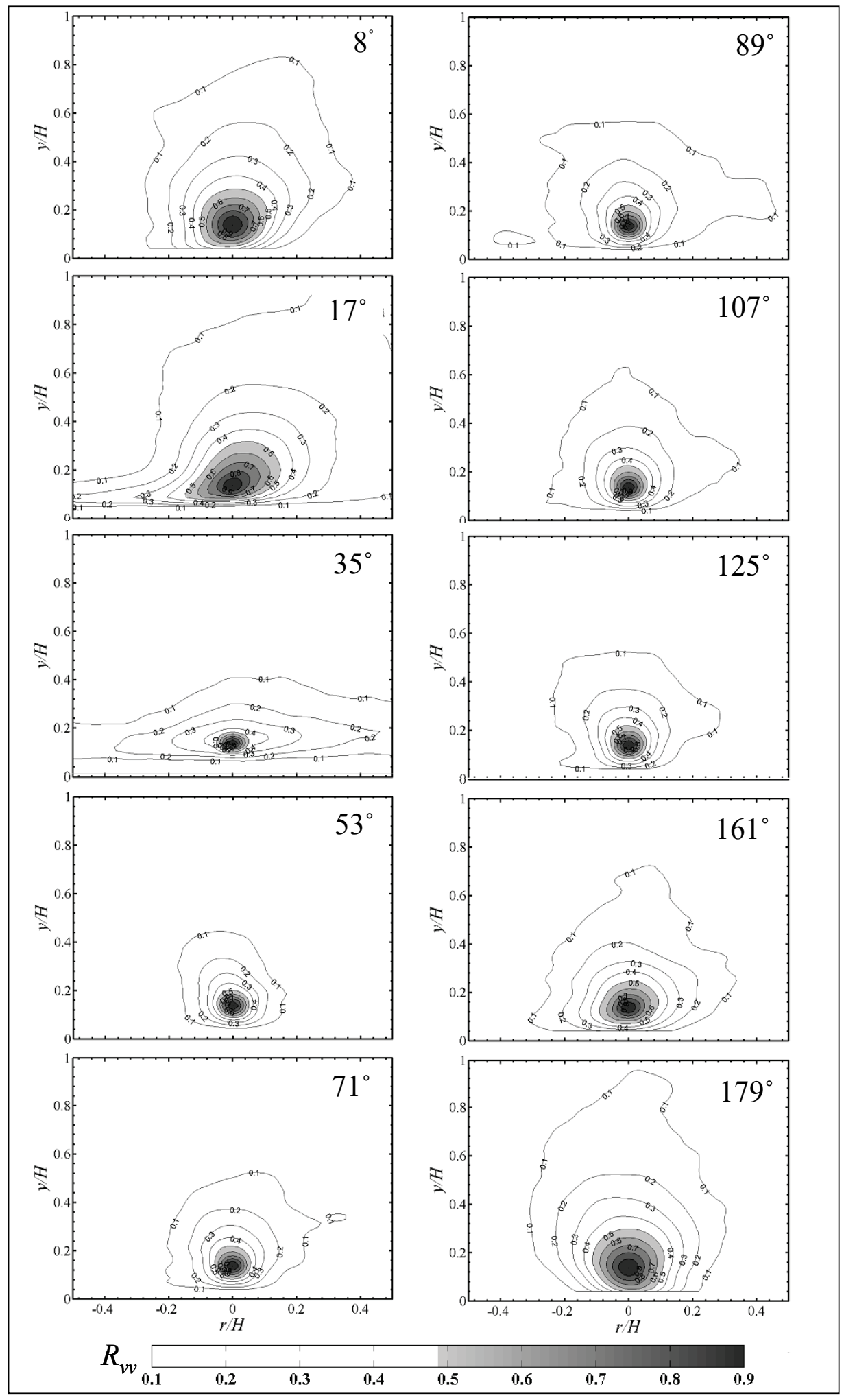


Early in the acceleration phase at $8^{\circ}$ and $17^{\circ}$, the turbulent fluctuations are correlated over very large spatial extents with the $R u$ contours exhibiting an inclination toward the upstream direction that persists from the previous half cycle where the bulk flow direction was reversed. The large spatial extent of both the streamwise and wall-normal velocity correlation fields is associated with the very large up- and downwash structures observed in the instantaneous velocity fields. The flow is relaminarized at $35^{\circ}$, where the extent of highly correlated $R_{u u}$ fills the channel half height with diminished size observed in $R_{v v}$, which can be interpreted to be a result of cycle-to-cycle jitter in the observed laminar plug flow with essentially zero vertical fluctuation. By $53^{\circ}$, turbulence production has restarted, and a highly elongated $R u u$ is observed, with no discernable inclination from the wall. This behavior is consistent with a turbulent boundary layer in the near-wall region with a laminar plug flow in the channel core. The elongated $R_{u u}$ correlation is also consistent with recent computational observations of the transition process in oscillating flows (Salon et al. 2007), where very long, streamwise-oriented streaky structures appear in the near-wall region at the initial onset of turbulence production. From $71^{\circ}$ to $125^{\circ}$, the $R_{u u}$ and $R_{v v}$ correlations are quantitatively similar to those reported by Christensen et al. (2004) for steady channel flows at $R e_{\tau}=1150$. In the $71^{\circ}$ to $125^{\circ}$ time frame, the flow in the channel is near the peak of the bulk velocity waveform shown in Figure 19, with minimal temporal change in pressure gradient and a nearconstant wall shear stress resulting in a value of $R e_{\tau}=900$. The $R_{u u}=0.5$ threshold is $\sim 0.9 \mathrm{H}$ in extent with an upward incline in $R u$ of $\sim 10^{\circ}-12^{\circ}$ in the data, compared to $12.5^{\circ}$ estimated from Christensens's (2004) results. The half cycle then ends with a growth of the extent of both $R_{u u}$ and $R_{v v}$, and the next half cycle then begins with this persistent large-scale turbulence.

\section{Conditional velocity fields}

Linear estimates of the evolution of the conditional velocity fluctuation fields for a $\lambda_{c i}$ event at $y_{o}=0.137 H$ (same as for velocity correlation data above) over the half cycle are shown in Figure 24. The half cycle begins with large-scale residual turbulence that persists from the previous deceleration phase; no appearance of the in-plane, hairpin-vortex signature of the type described by Adrian et al. (200ob) is observed until the late acceleration phase, with the first evidence of hairpin signatures appearing at a phase angle of $62^{\circ}$ (not shown here), where acceleration is near minimum but production of turbulence is still rising. 
Figure 24. Linear stochastic estimates of the expected velocity field given a $\lambda_{c i}$ event at $y_{\text {ref }} / H=0.137$ and $x / H=0$.

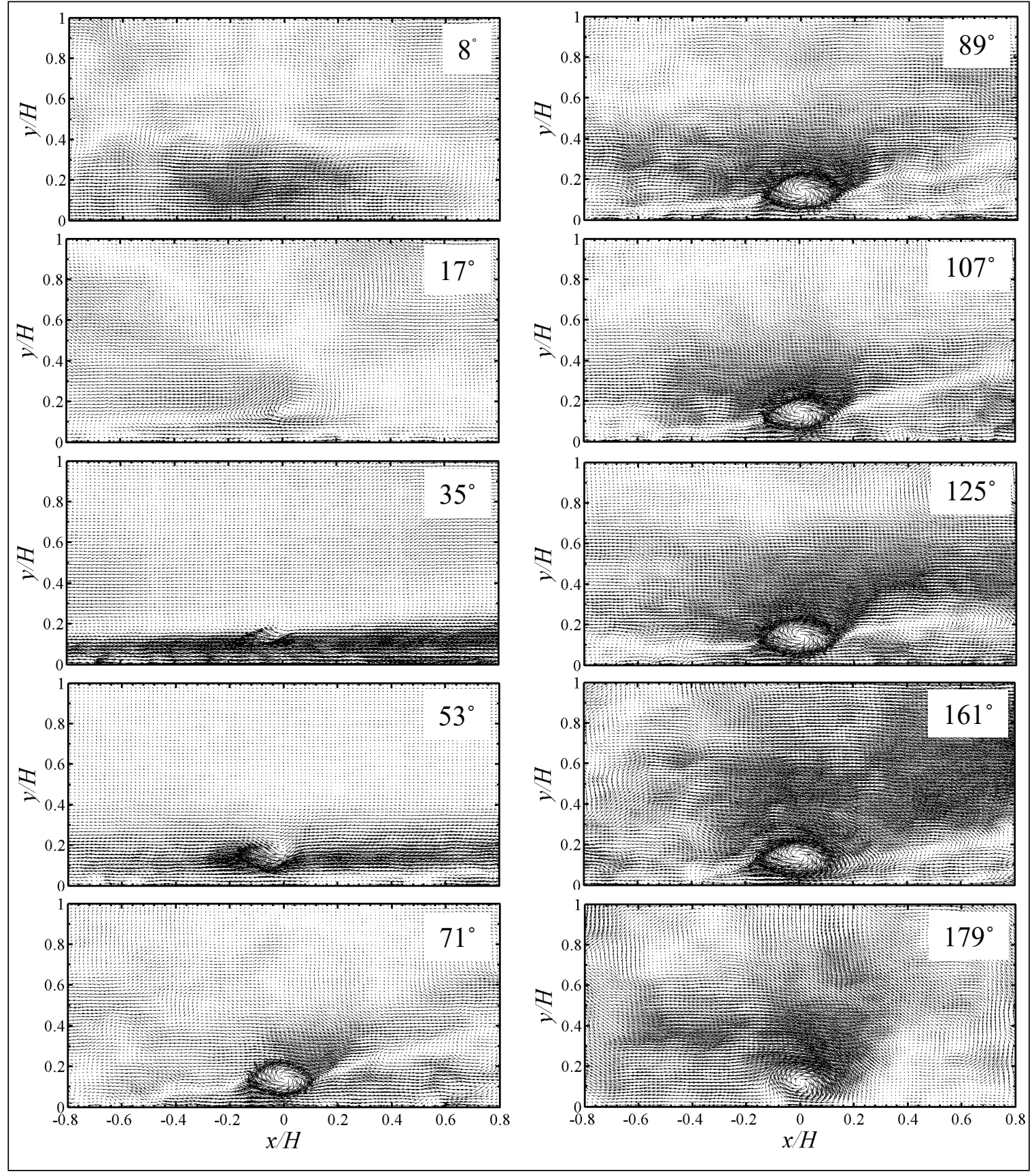

At phase angles from $71^{\circ}$ to $107^{\circ}$, the flow near the peak of the velocity waveform has achieved an essentially quasi-steady turbulent state, and the conditional velocity fields reveal a strong vortical circulation at the swirlevent location $\left(x=0, y=y_{0}\right)$ with other features indicative of the presence of hairpin vortices (Adrian et al. 200ob). A stagnation line in the reference frame of the vortex is inclined upward in the direction of the main flow 
and is present in all cases, with a large zone of uniform, low-momentum fluid underneath the stagnation line. This shallow inclination angle is similar to the feature observed in the $R$ ии contours of Figure 22 but larger at $\sim 16^{\circ}$. The vortex cores are reminiscent of hairpin vortex heads with Q2 events upstream of the vortex and associated Q4 events upstream above the stagnation line on the other side of the main vortex. Additional weaker vortex heads, revealed by a circular vector pattern along the inclined stagnation line, are observed downstream at nominal streamwise spacings of $\sim 0.4 \mathrm{H}$, suggesting that the hairpins are indeed traveling in packets convecting at approximately the same speed.

The hairpin-vortex signature then changes during the deceleration portion of the cycle. From $126^{\circ}$ through $161^{\circ}$, the inclination angle of the stagnation line is reduced although the presence of additional vortices at $\sim 0.4 \mathrm{H}$ spacing persists, until the end of the half cycle, where a singular, isolated vortex is observed without the additional features of hairpin vortex packets described above.

\section{Task 2-Create data base of shear-stress time histories by demonstrating and applying newly developed optical sensing technology within SEAWOLF}

\section{Optical shear-stress sensors demonstration}

A representative from Measurement Science Enterprise, Inc., performed a demonstration of their microS and miniLDV sensors in January 2009. Since these measurements were taken in 2009, a brief summary of findings is presented here while more detail will be presented in upcoming reports.

First, the microS sensor was tested under unidirectional flow conditions. The microS reported instantaneous values of shear stress in real time with the software provided. This allowed for analyzing the unidirectional data for the average shear stress as well as turbulent fluctuations. Several different unidirectional flows were established within the SEAWOLF, and for all cases, the average value of shear stress reported by the microS was within $10 \%$ of the value predicted by Blasius (Equation (14)). This indicated that the microS worked extremely well in fully developed unidirectional turbulent flow cases. 
Next, the microS sensor was tested under oscillatory flow conditions within the SEAWOLF flume. Several oscillatory cases were tested, but in all cases the quality of the data was limited by low data rate and by the limited dynamic range of the shear-stress sensor.

The miniLDV sensor was found to be a much better suited instrument for the measuring of wall shear stress using a similar single-point estimate technique than the microS sensor but with much improved data rate and sensor dynamic range. The miniLDV sensor is mounted externally to the SEAWOLF test section and is insensitive to particle fouling on sensor windows. In addition, frequency shifting electronics that remove the background pedestal from the Doppler burst signal are incorporated onboard the miniLDV, which provides for much improved sensor dynamic range that is a must for the wide variations in shear stress observed within the oscillating SEAWOLF flow. During the sensor demonstration, it was experimentally verified that the miniLDV velocity signal at several nearwall locations was linearly proportional to wall shear stress for unidirectional flow in SEAWOLF. A modified miniLDV sensor from MSE, with $\sim 30 \mu \mathrm{m}$ spatial resolution, was purchased, which will be used for detailed parametric studies of wall shear stress in Fiscal Year (FY) 09.

\section{Task 3-Demonstrate wave-current bottom shear-stress time histories representative of nature in SEAWOLF by manipulating flume and modulated flow conditions}

\section{Correlation of the wall shear-stress results}

Akhavan et al. (1991) has experimentally shown that the wall shear-stress measurements in oscillating pipe flow were well correlated with quasisteady applications of the Blasius formula (Equation (11)) during turbulent portions of the wave cycle. Akhhavan's laminar-phase, shear-stress data were additionally well correlated by laminar solution for oscillating channel flow. Comparisons of the measured wall shear-stress data with quasi-steady turbulent predictions were presented previously in this report. Results with the calculated shear-stress values were within $40 \%$ of the average shear and $27 \%$ of shear-stress maximum or minimum.

The shear stress imparted to a surface, or wall, by flowing water is given by Equation (3). With LDV, a single-point velocity measurement is made in a region near the wall in which $d u / d y$ is well approximated by a linear gradient. 


$$
\frac{d u}{d y} \approx \frac{u_{L D V}-u_{\text {wall }}}{y_{L D V}-y_{\text {wall }}}
$$

When the no-slip boundary condition, $u_{\text {wall }}=0$, is applied, and the viscosity to be a constant for this isothermal water flow is taken,

$$
\tau=\text { constant } \times \frac{u_{L D V}}{y_{L D V}-y_{\text {wall }}}
$$

so that the shear stress is proportional to the LDV-measured velocity when the LDV measurement volume is at a fixed distance, $y_{L D V}-y_{\text {wall, }}$ from the channel wall.

The LDV measurement was calibrated for shear stress by performing measurements in a steady SEAWOLF channel flow, where the wall shear stress was previously characterized by PIV and found to conform to wellknown results for turbulent channel flow. This results in an approximately linear calibration curve, as shown in Figure 25, where the measured $\tau$ vs. $u_{L D V}$ data are plotted against the predicted result for turbulent channel flow with $y_{L D V}-y_{\text {wall }}=112 \mu \mathrm{m}$. The slope of the measured calibration curve, as shown by the least-squares fit in the plot, is within $4 \%$ of the predicted result. Calibration experiments were performed before each LDV shear-stress experiment, and linear fits of the type shown in Figure 25 were used to convert measured LDV velocities to wall shear stress. Shearstress levels encountered in unsteady SEAWOLF channel flows were observed to be up to $6 \mathrm{~Pa}$, or twice the maximum shear present during calibration. The linear curves were extrapolated to these higher shear stresses with a maximum systematic error of $10 \%$, as estimated from lawof-the-wall velocity profiles for turbulent channel flow. 
Figure 25. Measured (symbols) and modeled (solid line) LDV shear-stress response in steady channel flow.

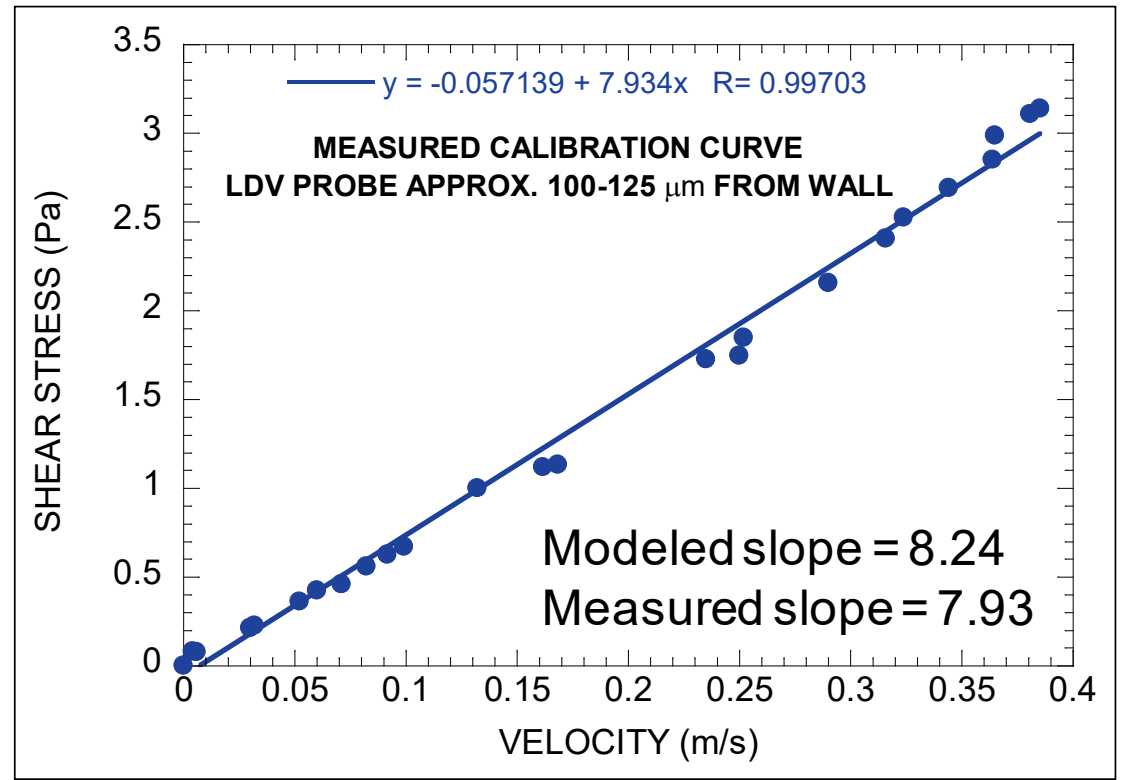

\section{Acquisition of initial LDV data sets and comparison of LDV- and PIV-} measured shear stresses

Phase-averaged shear stresses for six of the eight SEAWOLF operating conditions monitored by PIV have been measured using the LDV probe. The results for $5 \mathrm{sec}$ wave periods are shown in Figure 26, while the data for $10 \mathrm{sec}$ wave periods are displayed in Figure 27. Agreement between the PIV and LDV data is reasonable, with shear-stress amplitude generally within $\sim 30 \%$ for the two methods and similar shear-stress waveform shapes measured. The LDV probe generally yielded higher shear stresses than with PIV. In some cases, the measured shear-stress amplitudes are within 10\%. Two repeat LDV runs are plotted in Figure 28 for the case where the flow rate nominally varies between $+50 \mathrm{GPM}$ and $-50 \mathrm{GPM}$ at $10 \mathrm{sec}$ wave period. The repeatability of the LDV measurements is excellent. 
Figure 26. Comparison of LDV- and PIV-measured shear stresses for 5 sec SEAWOLF wave period.

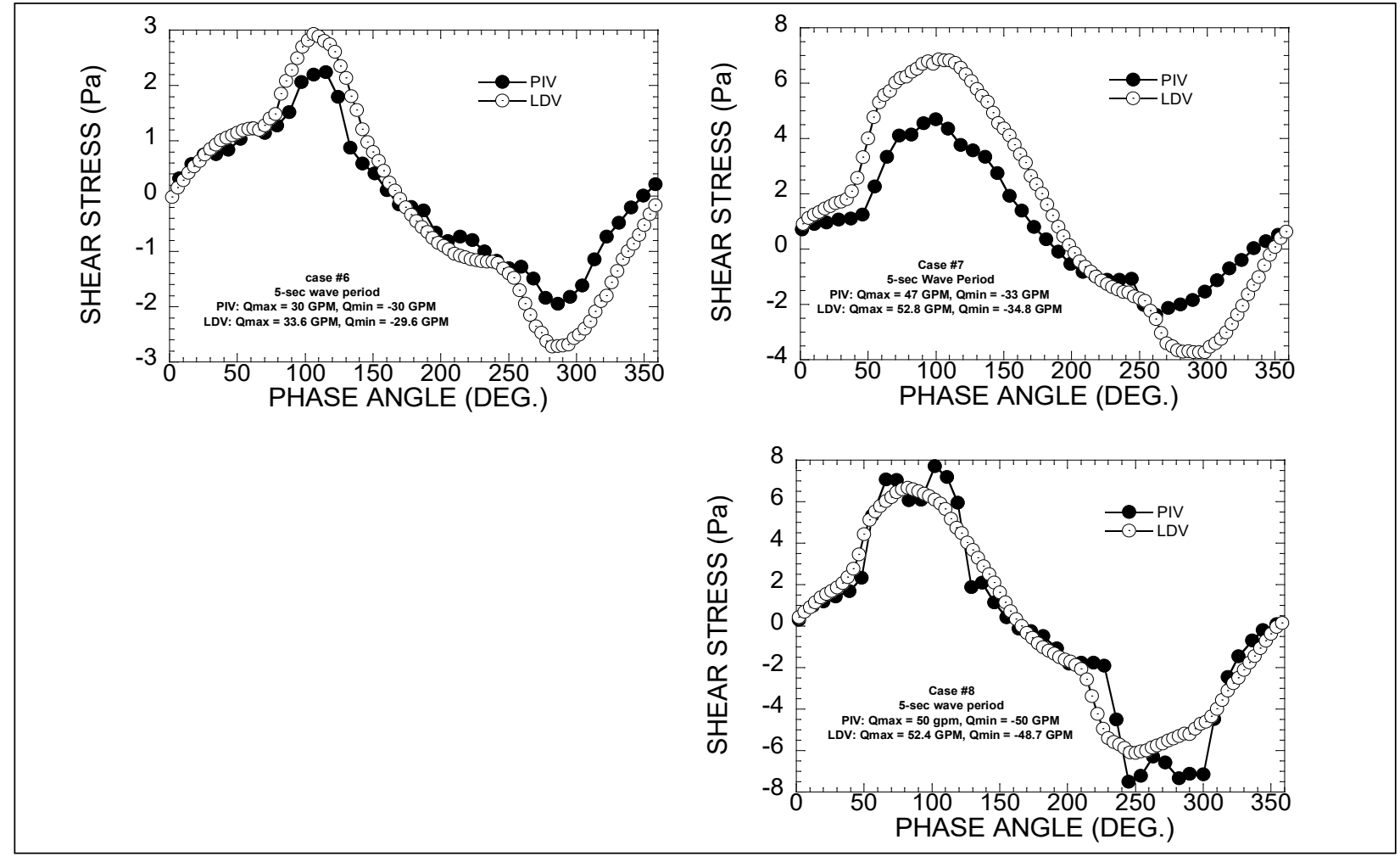

Figure 27. Comparison of LDV- and PIV-measured shear stresses for 10 sec SEAWOLF wave period.

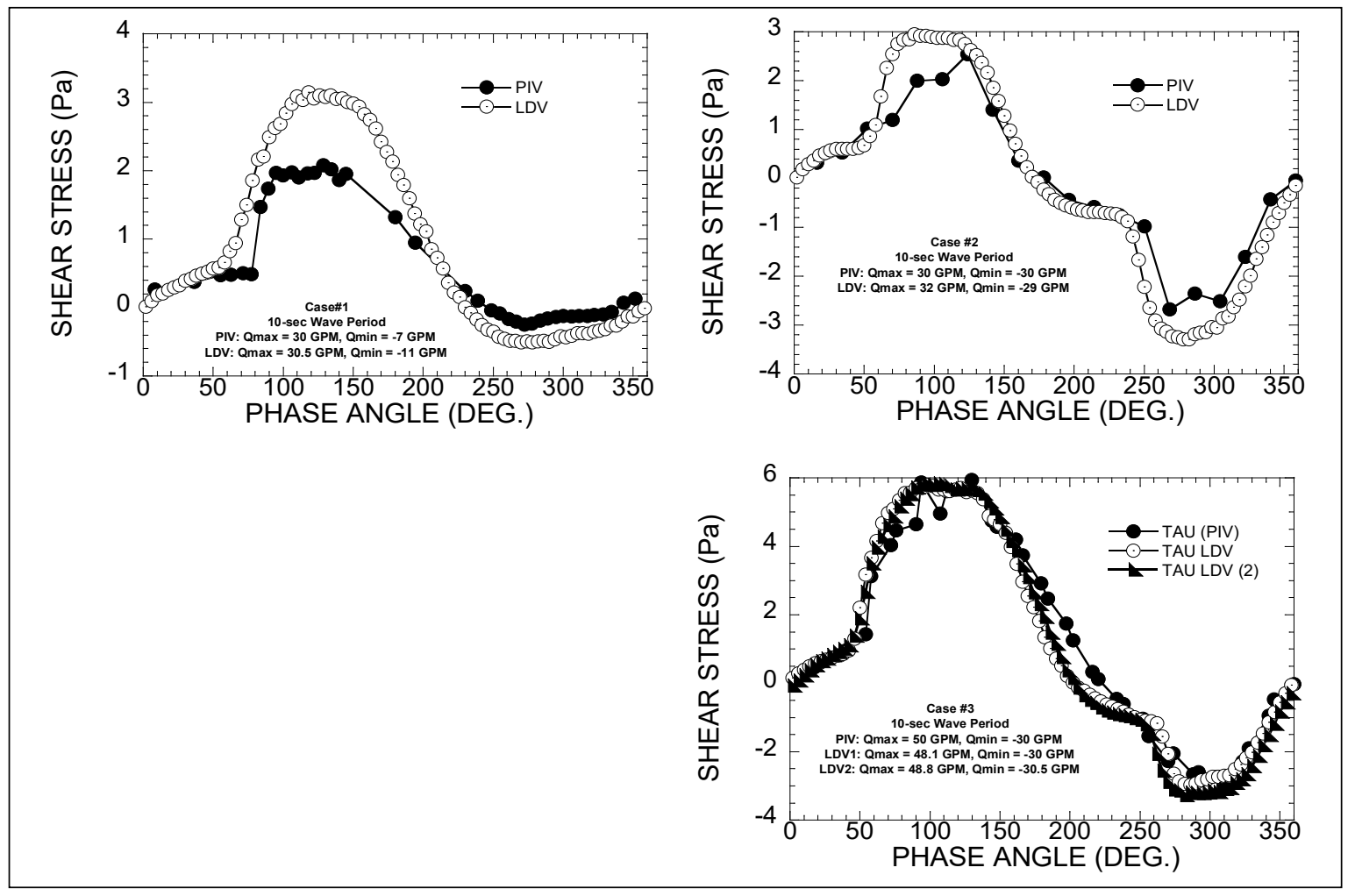




\section{Measurement of instantaneous shear-stress fluctuations by LDV}

One major advantage of LDV probing of shear stress is that data are acquired on an instantaneous basis, versus a phase-averaged basis as with PIV. In other words, only the average shear-stress wave cycle is measured with PIV while individual wave cycles can be resolved with the LDV sensor. An example instantaneous shear-stress wave form is shown in Figure 28 for a case with maximum and minimum flow rates of 30.2 and 24.45 GPM and $10 \mathrm{sec}$ wave period. Approximately 4 cycles of a $200+$ cycle data set are shown on the plot. In contrast to the data shown in Figures 26 and 27, where only the phase-averaged waveform is shown, the instantaneous shear-stress wave reveals strong turbulent fluctuations superposed with the phase average. Because sediment erosion rate is often modeled as power-law behavior,

$$
E=B \tau^{n}
$$

where $E$ is the instantaneous sediment erosion rate, $B$ is an empirical constant. The exponent, $n$, is an empirically determined exponent and is frequently found to be greater than 2 so that large turbulent fluctuations have a significant effect, with an impact that is amplified by the strong nonlinear relationship between $E$ and $\tau$. LDV measurements of the kind shown in Figure 28 offer the possibility of quantifying these strong erosionenhancing events, while PIV, in the present implementation, cannot.

Figure 28. Instantaneous shear-stress wave form measured using LDV in SEAWOLF.

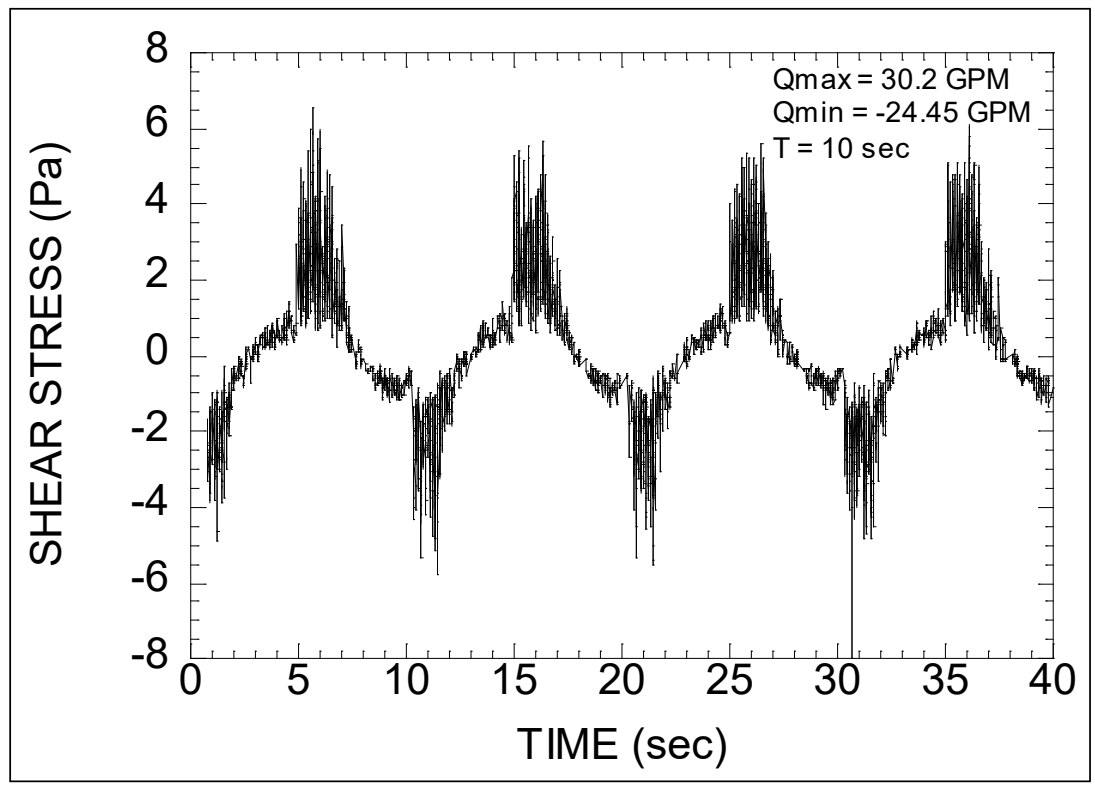


Shear-stress time histories of the type shown in Figure 29 can be phasedecomposed to quantify the shear stress excursions. Here, the instantaneous shear stress at any position, $\mathbf{x}$, can be expressed as an average waveform, $\langle\tau\rangle$, and an instantaneous fluctuation, $\tau^{\prime}$, about $\langle\tau\rangle$ :

$$
\tau(\mathbf{x}, t)=\langle\tau\rangle(\mathbf{x}, \theta)+\tau^{\prime}(\mathbf{x}, t)
$$

Note that $\tau$ and $\tau$ ' are functions of time, while $\langle\tau\rangle$ is a function of the cycle phase angle, $\theta$. At each $\theta$ there can be a computation of an rms fluctuation of $\tau$ about $\langle\tau\rangle$. These $\tau_{r m s}$ quantify an average strength of the shear-stress fluctuations at any cycle phase angle, and the shear-stress fluctuations computed from the full $200+$ cycle data set, of which a subset is shown in Figure 28, is shown in Figure 29. The baseline shear-stress fluctuation is approximately $0.2 \mathrm{~Pa}$. At these cycle times, the flow is relaminarized, and the baseline fluctuations are likely due to small levels of jitter in the cycleto-cycle flow rate waveform and by measurement error. The rms shear stress experiences abrupt increases to maximum values as a result of transition to turbulence and decays as the flow is decelerated. The flow changes direction at a phase-averaged cycle time of $\sim 5 \mathrm{sec}$ and is relaminarized again before the process is repeated in the negative flow rate portion of the cycle.

While rms shear-stress does a good job of quantifying the average level of turbulent shear-stress fluctuation throughout the wave cycle, it does not capture the maximum shear-stress excursions. To address this issue, probability densities (histograms) of the instantaneous shear-stress measurements have been computed. A representative histogram is shown on the right-hand side of Figure 30, as computed from the data set of Figure 28. Here, maximum shear-stress excursions of $5 \mathrm{~Pa}$ (Figure 29, upper right corner) are observed; it is the tails of these shear-stress histograms that can lead to significant unsteady erosion events. 
Figure 29. Representative LDV shear-stress data sets for two different flow rate conditions at 10 sec wave periods.

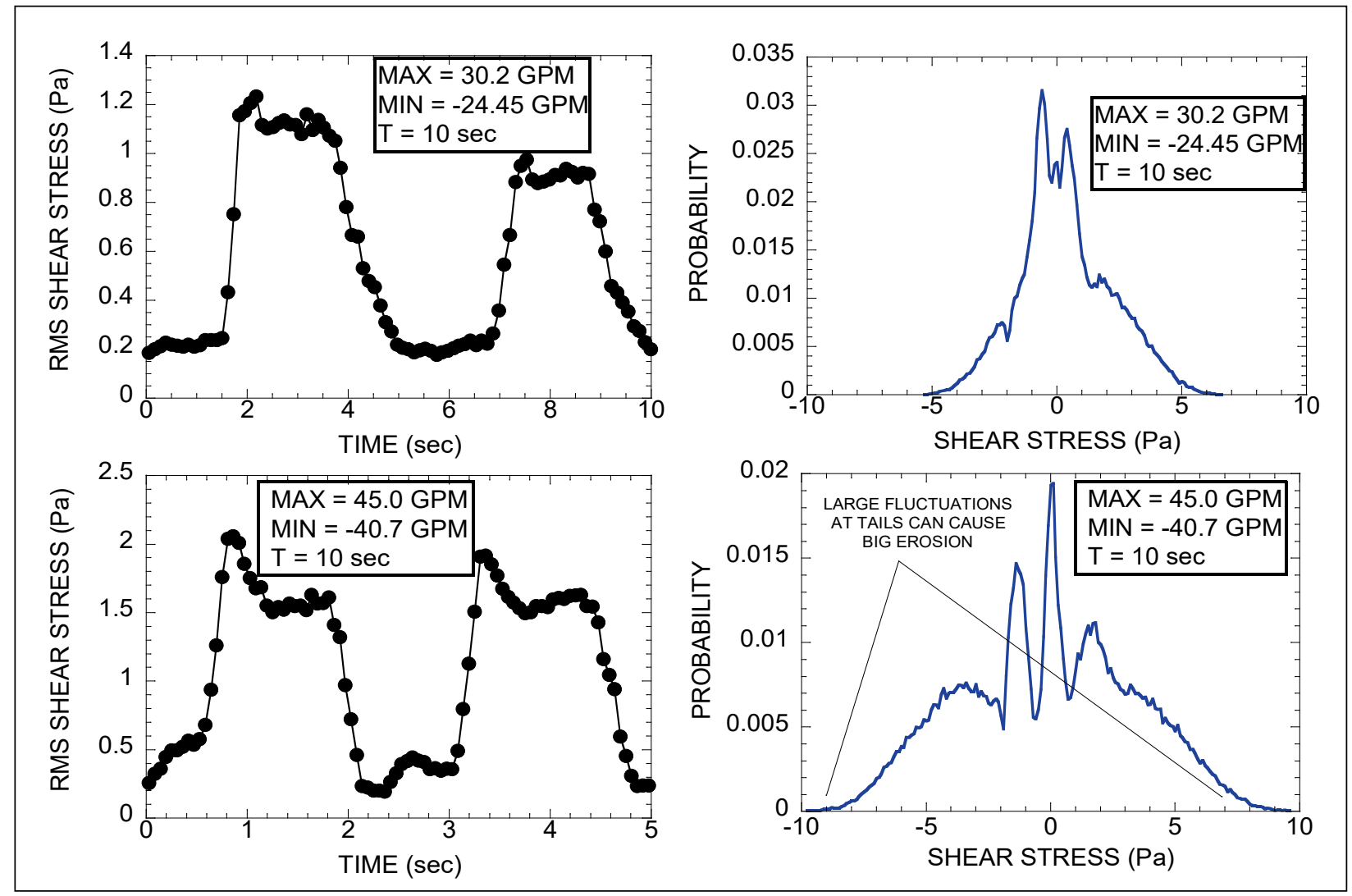

\section{Parametric study of shear-stress waveforms generated by SEAWOLF}

The LDV sensor in production mode testing has been applied to investigate the shear-stress waveforms provided by SEAWOLF under a range of wave and current flow rates and for wave periods of 5 and $10 \mathrm{sec}$. The waveforms were generated by varying the following controls:

1. The programming of the SEAWOLF pistons in the Anaheim automation software; four different programmed piston motions were run
a. "50_10": a 10 sec wave period with a piston stroke of 8.17 in.
b. "50_5": a $5 \mathrm{sec}$ wave period with a piston stroke of $4.1 \mathrm{in}$.
c. "40_10": a 10 sec wave period with a piston stroke of 6.54 in.
d. "40_5": a $5 \mathrm{sec}$ wave period with a piston stroke of $3.27 \mathrm{in}$.

2. The position of the flow metering valve
a. Fully open
b. $45^{\circ}$ to the horizontal
c. $60^{\circ}$ from the horizontal 
3. With the water pump that provides DC or steady current flow on or off

With these parameters in mind, each of the test cases was assigned a unique name so that it was obvious what the SEAWOLF control settings were for each experiment. An example of this naming convention for an operating condition running the "50_5" SEAWOLF piston program with the metering valve position at $45^{\circ}$ to the horizontal and the water pump off is

$$
\text { 50_5_45_off }
$$

All data sets were named in a similar manner, with a total of 22 different combinations investigated (Table 3).

Table 3. Summary of peak phase-averaged flow and shear-stress data in SEAWOLF.

\begin{tabular}{|l|l|l|l|l|l|}
\hline Title & T (sec) & Qmax (GPM) & Qmin (GPM) & $\tau \operatorname{max~(Pa)~}$ & $\tau$ min (Pa) \\
\hline 50_5_45_on & 5 & 50.8 & -29.6 & 6.32 & -2.87 \\
\hline 50_5_60_on & 5 & 52.46 & -41.4 & 6.39 & -5.15 \\
\hline 50_5_open_off & 5 & 31.3 & -29.37 & 2.01 & -2.58 \\
\hline 50_5_45_off & 5 & 43.4 & -40.84 & 4.9 & -5.45 \\
\hline 50_5_60_off & 5 & 48.7 & -44.97 & 5.66 & -5.93 \\
\hline 50_10_open_on & 10 & 57.15 & -17.44 & 7.37 & -0.8323 \\
\hline 50_10_45_on & 10 & 55.71 & -35.06 & 6.98 & -4.46 \\
\hline 50_10_open_off & 10 & 35.65 & -33.48 & 3.71 & -4.09 \\
\hline 50_10_45_off & 10 & 41.18 & -38.75 & 4.72 & -5.15 \\
\hline 50_10_60_off & 10 & 47.14 & -44.51 & 5.36 & -5.78 \\
\hline 40_10_45_off & 10 & 32.37 & -31.09 & 3.38 & -3.77 \\
\hline 40_10_45_on & 10 & 49.95 & -20.23 & 5.57 & -1.23 \\
\hline 40_10_60_on & 10 & 46.92 & -29.51 & 5.2 & -2.96 \\
\hline 40_10_open_off & 10 & 26.8 & -25.94 & 2.26 & -2.37 \\
\hline 40_10_open_on & 10 & 52.66 & -7.07 & 5.53 & -0.1525 \\
\hline 40_5_open_on & 5 & 48.26 & -3.2 & 5.58 & 0.0118 \\
\hline 40_5_45_on & 5 & 44.71 & -27.04 & 5.15 & -2.38 \\
\hline 40_5_60_on & 5 & 43.25 & -33.91 & 4.9 & -3.77 \\
\hline 40_5_open_off & 5 & 23.42 & -22 & 0.7448 & -0.8644 \\
\hline 40_5_45_off & 5 & 29.33 & -28.09 & 1.94 & -2.57 \\
\hline 40_5_60_off & 5 & 37.51 & -35.13 & 3.49 & -3.93 \\
\hline 50_5_open_on & 52.79 & -12.39 & 6.29 & -0.61 \\
\hline & 5 & & & & \\
\hline
\end{tabular}


The measurements were carried out with the LDV probe volume positioned approximately 80 to $120 \mu \mathrm{m}$ from the floor of the SEAWOLF test section. At this location, the probe was between $y^{+}$of 4 to 10 viscous wall units from the wall for instantaneous shear stresses ranging from 1 to $6 \mathrm{~Pa}$, respectively. For $5<y^{+}<10$, the probe is outside the viscous sublayer and instead, is in the lower portions of the buffer layer, where small departures in linearity in the velocity profile are present. Using the well-known law-of-the-wall for turbulent velocity profiles, a maximum systematic error of $10 \%$ for a phaseaveraged wall shear stress of $6 \mathrm{~Pa}$ is estimated. For each operating condition, the LDV was calibrated for its shear-stress sensitivity, and a curve similar to the one shown in Figure 25 was generated. Oscillating flow was then generated in SEAWOLF, and LDV data were recorded continuously for a period between 20 and 30 minutes, resulting in 100 to 300 wave cycles of instantaneous LDV data. The calibration was repeated after each run as well so that any drift in the velocity/shear-stress calibration could be monitored over the course of the day's testing. The calibration was then applied to the raw LDV data, and the results were phase averaged as per Equation (5). For each of the 22 operating conditions studied, there is a presentation of the phase-mean flow rate, phase-mean shear-stress, phase-locked rms shear-stress fluctuation (the fluctuation about the phase mean), and a 10-wave-period sample of the instantaneous fluctuations.

Sample results for the case "40_10_open_off" are shown in Figure 30, with the data for the remaining 21 operating conditions provided in Appendix A. In Figure 30, the phase-averaged flowrate (i.e., the average flow rate cycle) is plotted at the upper left with the corresponding phase-averaged, shearstress waveform at the upper right. The rms value of the phase-locked turbulence, $\tau$ ' as per Equation (5), is plotted at lower left, and 10 cycles of the instantaneous LDV data are at lower right to illustrate the degree of turbulent fluctuation in the flow. Note that hardware for locking the flow meter and LDV probe was available during these experiments, and the zero time of the flow and shear-stress mean and rms waveforms was adjusted so that the positive-running zero crossing was approximately at time $=0$. This was done to provide an approximate degree of correlation between the flow and shear-stress data, but the phase relationship between these two quantities is not exact. Transition to turbulence with the associated profound impact on shear stress can be identified in each data set by a sudden spike in the degree of scatter in the instantaneous shear-stress data, which is accompanied by a sudden rise in the phase-averaged shear-stress and rms shear-stress fluctuation. 
Figure 30. Phase-averaged data for operating condition 40_10_open_off.

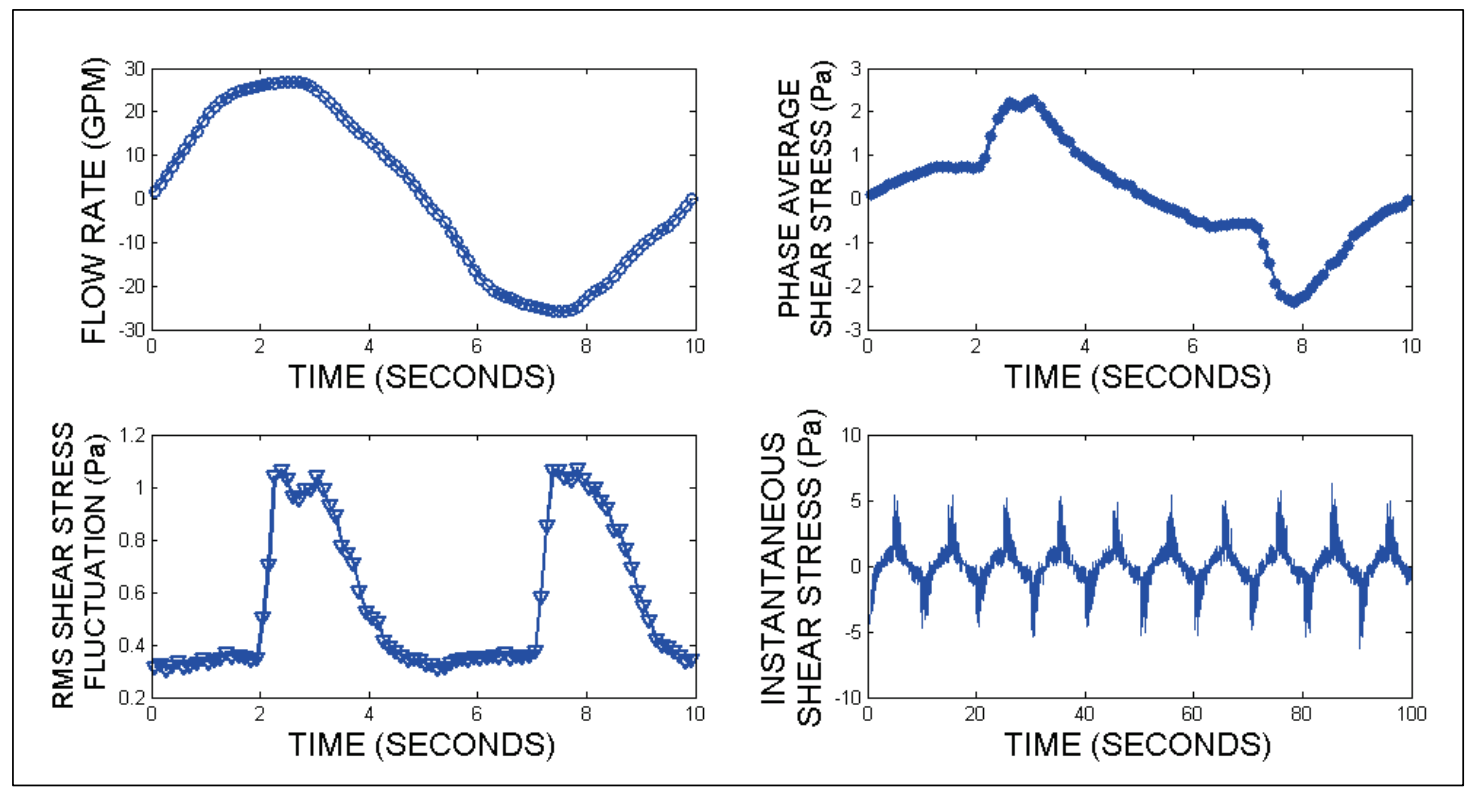

Results for all 22 cases investigated in the parametric study are listed in Table 3 in terms of the name designation of the data set, the wave period $(T)$, phase-averaged minimum and maximum flow rates $\left(Q_{\min }\right.$ and $\left.Q_{\max }\right)$, and phase-averaged minimum and maximum shear stress ( $\tau_{\min }$ and $\tau_{\max }$ ). The cycle minimum and maximum shear stresses were plotted against the corresponding minimum and maximum flow rates for all 22 data sets, with the results shown in Figure 31. The results for the cycle maximum (peak or positive flow rate) are plotted in blue, with the data for the cycle minimum (trough or negative flow rate) plotted in green symbols. Both sets of data are well correlated by the Blasius shear-stress equation for steady turbulent channel flow:

$$
\tau_{w}=0.0396 \rho U^{7 / 4}\left(\frac{v}{d}\right)^{1 / 4}
$$

and this level of agreement is consistent with the quasi-steady nature of the SEAWOLF flow. In Equation (34), $\tau_{w}$ is the wall shear stress, $\rho$ is the water density, $U$ is the volume-integrated average velocity, $d$ is the channel hydraulic diameter, and $v$ is the kinematic viscosity of water. This result shows that the Blasius equation can be used as a first-order predictor of the amplitude of the phase-locked shear stress based upon the programmed SEAWOLF flow rate waveform. 
Figure 31. Cycle minimum and maximum phase-averaged shear stresses correlated with their corresponding minimum and maximum phase-averaged flow rates.

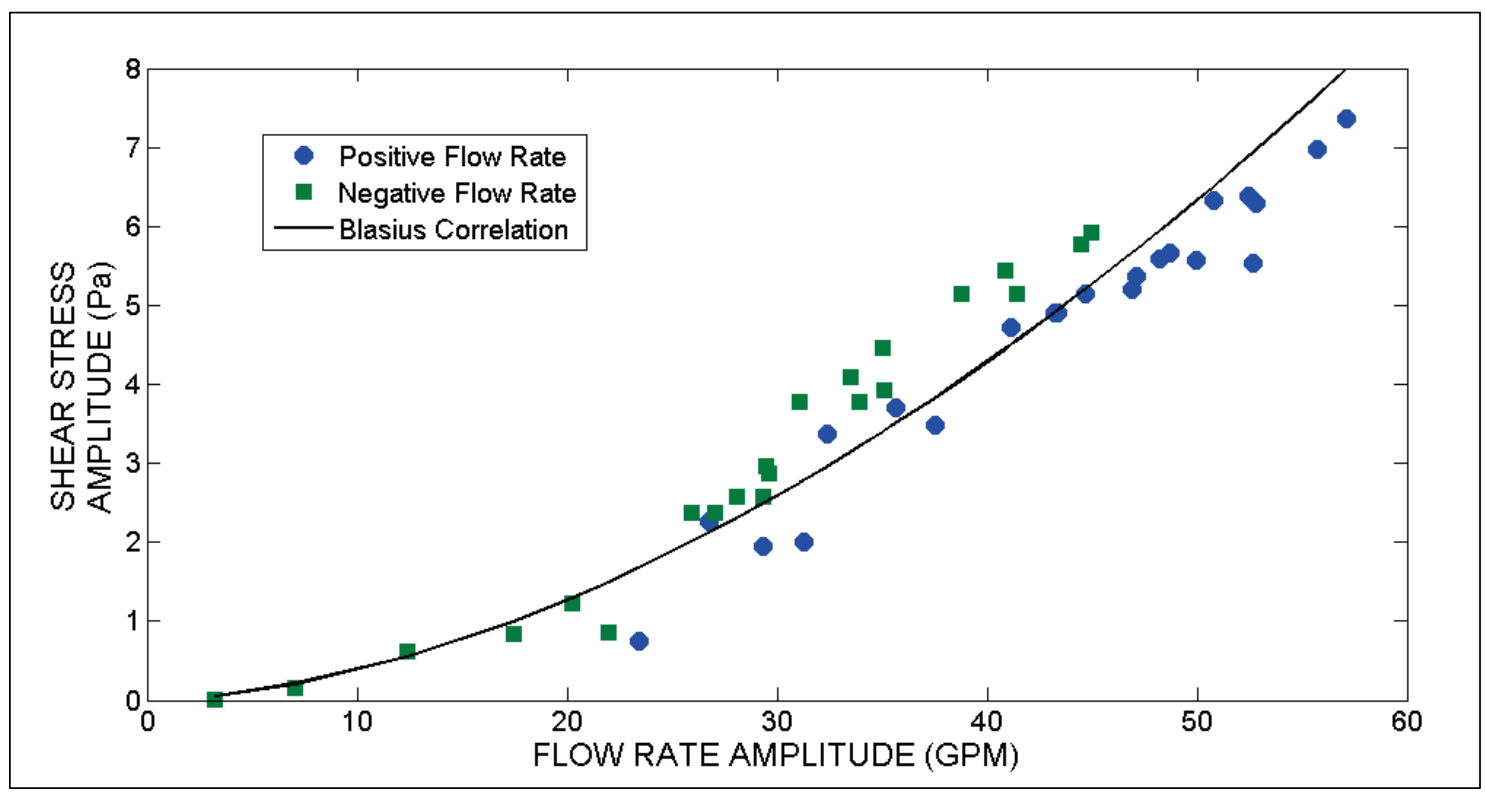

\section{Task 4-Investigate uncertainty in shear-stress measurement techniques}

The wall shear stress was inferred from high-magnification PIV measurements of the velocity profile in the viscous sublayer using Equation (3). The impact of two primary causes of measurement error has been considered: (1) uncertainty in the viscosity, $\mu$, resulting from temperature variations during the approximate 4-hour duration of a given measurement run, and (2) the confidence interval assigned to the leastsquares estimate of the slope of the velocity data, $d u / d y$, in the viscoussublayer region. For a $\pm 2{ }^{\circ} \mathrm{C}$ variation in temperature over the course of a given data run, the water viscosity was found to vary by $\pm 5 \%$. The larger source of measurement error is associated with the confidence interval associated with the least-squares estimate of $d u / d y$. In general, the fitted slope from linear fitting of the sublayer data represents the true value of $d u / d y$ within an interval of $\pm t \sqrt{C}$, where $t$ is the student's $t$ multiplier for a 95\% level of confidence and $C$ are elements of a covariance matrix which measures the difference between the measured velocity data and the velocities predicted from the least-squares fit. In general, the confidence interval improves (get smaller) when more data points are available for fitting and as the signal-to-noise in the PIV-measured velocity profile improves. These confidence intervals from the available PIV data have been calculated, and it is found that the uncertainty in $d u / d y$ is generally within $\pm 20 \%$ when four or more points are available for fitting within the 
viscous sublayer. Based on these results, and the degree of scatter observed in the measured shear-stress time histories, it is felt that the data are uncertain to within $\pm 20 \%$ to $25 \%$ or better. 


\section{Concluding Summary}

\section{Task 1-Determine shear-stress time histories and turbulent structure within the enclosed SEAWOLF channel with smooth and rough walls under modulated flow}

\section{Cyclic modulation of wall shear-stress and turbulent properties in oscillating channel flow within the SEAWOLF flume}

A hybrid-magnification PIV instrument with automated dynamic-range adjustment for the study of the phase modulation of the wall shear stress and flow structure in an oscillating channel flow has been applied. A highmagnification PIV setting was utilized to obtain superior resolution of the near-wall region, with an in-plane spatial resolution ranging from 0.2 to 1.6 viscous wall units, which enabled high-resolution observation of the phase modulation of the viscous and logarithmic regions of the velocity profile in oscillating flow. At $R e_{\delta}=1220$, it was observed that relaminarization inhibits the formation of a logarithmic velocity profile until well into the later portions of the velocity half cycle. Fitting of late-cycle velocity data at $\operatorname{Re}_{\delta}=1220$ to a logarithmic curve with $\kappa=0.41$ reveals that the constant $B$ decays from $\sim 6.5$ at the inception of logarithmic behavior to a value near 5.0 at the end of the half cycle. Fits to the mean velocity data in the viscous sublayer were used to obtain reliable wall shear-stress measurements, which were within $\sim 10 \%-15 \%$ of shear-stress estimates obtained from an accepted empirical correlation and fitting to log-layer velocity data in a steady flow test case. A low-magnification PIV setting was used to reveal the flow structure across the full channel height, revealing the instantaneous flow structure during relaminarization and transition at $R e_{\delta}=1220$ and quantifying the phase-evolution of the channel-integrated turbulent kinetic energy and production rate at $R e_{\delta}=1220,2033$, and 2875. The results quantify the impact of transition and relaminarization on wall shear stress and quantify the decrease in the duration of the laminar phase of the cycle by about a factor of 2 for $\operatorname{Re}_{\delta}$ from 1220 to 2875 .

\section{Turbulence structure in oscillating channel flow}

A detailed analysis of the structure of turbulence in an oscillating channel flow at $\operatorname{Re}_{\delta}=2033$ using PIV data in the streamwise/wall-normal plane has been presented. To the authors' knowledge, this is the first structural information for an oscillating flow derived from direct velocity measurements. The phase evolution of the two-point spatial correlation functions, 
$R_{u u}$ and $R_{v v}$, along with linear stochastic estimates of the velocity field in the presence of a swirling strength event in the log layer are presented. The results reveal a flowfield that is dominated by very large residual turbulence events in the early phases of the cycle acceleration phase, which is followed by a brief period of relaminarized flow in the presence of a strong acceleration. Turbulence re-emerges in the later portions of the cycle acceleration phase, where the weakly accelerated/decelerated flow in the vicinity of the peak bulk velocity attains a quasi-steady turbulent state with evidence of hairpin-like structures resembling those reported in steady turbulent wall flows. These hairpin-like vortices are observed to persist through much of the deceleration phase of the half cycle, vanishing only just before the end of the half cycle where flow reversal and a change in the sign of the acceleration occur in the near-wall region.

\section{Task 2-Create data base of shear-stress time histories by demonstrating and applying newly developed optical sensing technology within SEAWOLF}

\section{Optical shear stress sensor demonstration}

As an alternative to laborious PIV shear-stress measurements, two newly developed commercial optical sensor technologies were tested, the microS and miniLDV, which (1) offer calibration-free measurements, (2) rapid interrogation of near bed velocities for wall shear stress, and (3) have been proven to work in harsh and dirty environments. The miniLDV significantly outperformed the microS in initial testing and therefore was implemented within the SEAWOLF facility.

\section{Task 3-Demonstrate wave-current bottom shear-stress time histories representative of nature in SEAWOLF by manipulating flume and modulated flow conditions}

Comparisons of the measured wall shear-stress data with quasi-steady turbulent predictions were presented. Results with the calculated shearstress values were within $40 \%$ of the average shear and $27 \%$ of shear-stress maximum or minimum. While this may seem high, shear-stress fluctuations in natural systems are typically of a similar range. A total of 22 parametric flow-rate period and amplitude experiments were conducted to generate an empirical data base that can be used to program the SEAWOLF facility for a desired shear-stress waveform to be used in erosion testing of sediment core samples. The parametric data were generated for wave periods of 5 and 10 
sec with flow-rate amplitudes ranging from 5 to 60 GPM for cases with and without unidirectional current flow. The results reveal the complex periodically unsteady laminar-to-turbulent transition followed by relaminarization processes in SEAWOLF, with peak shear-stress amplitudes primarily occurring in the turbulent regime and well correlated to peak flow rates using the well-know Blasius correlation for steady turbulent channel flow. Results indicate that the Blasius equation (Equations (19) and (20)) can be used as a first-order predictor of the amplitude of the phase-locked shear stress based upon the programmed SEAWOLF flow rate waveform. This data set can now be used in a practical fashion to provide shear-stress time histories for erosion testing.

\section{Task 4-Investigate uncertainty in shear-stress measurement techniques}

Consideration has been given to the impact of two primary causes of measurement error: (1) uncertainty in the viscosity, $\mu$, resulting from temperature variations during the approximate 4-hour duration of a given measurement run and (2) the confidence interval assigned to the leastsquares estimate of the slope of the velocity data, $d u / d y$ in the viscoussublayer region. For $\mathrm{a} \pm 2{ }^{\circ} \mathrm{C}$ variation in temperature over the course of a given data run, the water viscosity was found to vary by $\pm 5 \%$. The larger source of measurement error is associated with the confidence interval associated with the least-squares estimate of $d u / d y$. Calculation of confidence intervals from the available PIV data demonstrate that the uncertainty in $d u / d y$ is generally within $\pm 20 \%$ when four or more points are available for fitting within the viscous sublayer. Based on these results, and the degree of scatter observed in the measured shear-stress time histories, it is felt that the data are uncertain to within $\pm 20 \%$ to $25 \%$ or better. 


\section{References}

Adrian, R. J., K. T. Christensen, and Z. C. Liu. 2000a. Analysis and interpretation of instantaneous turbulent velocity fields. Experiments in Fluids 29:275-290.

Adrian, R. J., C. D. Meinhart, and C. D. Tomkins. 200ob. Vortex organization in the outer region of the turbulent boundary layer. Journal of Fluid Mechanics 422:1-54.

Adrian, R. J. 2007. Hairpin vortex organization in wall turbulence. Physics of Fluids 19:041301.

Akhavan, R., R. D. Kamm, and A. H. Shapiro. 1991. An investigation of transition to turbulence in bounded oscillatory stokes flows, Part 1. Experiments. Journal of Fluid Mechanics 225:395-422.

Bijker, E. W. 1971. Longshore transport computations. Journal of Waterways, Harbors, and Coastal Engineering Division 97(WW4):687-701.

Borrowman, T. D., E. R. Smith, J. Z. Gailani, and L. Caviness. 2006. Erodibility study of passaic river sediments using USACE SEDflume. ERDC TR-06-7. Vicksburg, MS: U.S. Army Engineer Research and Development Center.

Cantwell, B. J. 1981. Organized motion in turbulent flow. Annual Review of Fluid Mechanics 13:457-515.

Christensen, K. T., Y. Wu, R. J. Adrian, and W. Lai. 2004. Statistical imprints of structure in wall turbulence, AIAA 2004-1116. Presented at the 42nd Aerospace Sciences Meeting and Exhibit, Reno, NV, 5-8 January 2004.

Christensen, K. T., and R. J. Adrian. 2001. Statistical evidence of hairpin vortex packets in wall turbulence. Journal of Fluid Mechanics 431:433-443.

Clauser, F. H. 1956. The turbulent boundary layer. Advances in Applied Mechanics 4:151.

Costamagna, P., G. Vittori, and P. Blondeaux. Coherent structures in oscillatory boundary layers. Journal of Fluid Mechanics 474:1-33.

Currie, I. G. 1993. Fundamental mechanics offluids, 2nd ed. New York: McGraw-Hill.

DaVis 7.2. 2006. Product manual. Göttingen, Germany: LaVision Inc.

Dibajnia, M., and A. Watanabe. 1992. Sheet flow under nonlinear waves and currents. In Proceedings of 23rd Coastal Engineering Conference, ASCE, 2015-2028. Reston, VA.

Fishler, L. S., and R. S. Brodkey. 1991. Transition, turbulence, and oscillating flow in a pipe. Experiments in Fluids 11:388-398. 
Fourguette, D., P. Gonzalez, D. Modarress, E. Arek, D. Wilson, and M. Koochesfahani. 2004. Optical measurement of wall shear stress with emphasis on flows near separation, AIAA2004-2394. 24 $4^{\text {th }}$ AIAA Aerodynamic Measurement Technology and Ground Testing Conference, Portland, OR.

Gailani, J., S. Smith, M. Channell, R. Jepsen, and J. Roberts. 2001. Sediment erosion study for the Housatonic River. Report prepared by the U.S. Army Engineer Research and Development Center, Vicksburg, MS. Concord, MA: U.S. Army Corps of Engineers District, New England.

Grant, W., and O. Madsen. 1979. Combined wave and current interaction with a rough bottom. Journal of Geophysical Research 84(C4):1797-1808.

Hino, M., M. Kashiwayanagi, A. Nakayama, and T. Hara. 1983. Experiments on the turbulence statistics and the structure of a reciprocating oscillatory flow. Journal of Fluid Mechanics 131:363-400.

Hinze, J. O. 1987. Turbulence, $2^{\text {nd }}$ ed. New York: McGraw-Hill.

Jensen, B. L., B. M. Sumer, and J. Fredsoe. 1989. Turbulent oscillatory boundary layers at high Reynolds numbers. Journal of Fluid Mechanics 206:265-297.

Jepsen, R., J. Roberts, and W. Lick. 1997. Effects of bulk density on sediment erosion rates. Water, Air, and Soil Pollution 99:21-31.

Jepsen, R., J. Roberts, and J. Gailani. 2004. Erosion measurements in linear, oscillatory, and combined oscillatory and linear flow regimes. Journal of Coastal Research 20(4):1096-1101

Jepsen, R., J. Roberts, J. Z. Gailani, and S. Smith. 2002. The SEAWOLF flume: Sediment erosion actuated by wave oscillations and linear flow. Sandia Report, SAND2002-0100, January 2002. Albuquerque, NM: Sandia National Laboratories.

Jepsen, R., J. Roberts, and J. Gailani. 2010. Effects of bed load and suspended load on separation of sands and fines in mixed sediment. Journal of Waterway, Port, Coastal, and Ocean Engineering 136(6):319-326.

Kearney, S. P., T. J. O'Hern, T. G. Dimiduk, T. W. Grasser, J. Barney, and J. D. Roberts. 2008. Particle-image velocimetry investigation of an oscillating turbulent channel flow, AIAA 2008-693. Presented at the $46^{\text {th }}$ AIAA Aerospace Sciences Meeting and Exhibit, Reno, NV.

Kendall, A., and M. Koochesfahani. 2006. A method for estimating wall friction in turbulent boundary layers, AIAA 2006-3834. Presented at 25 $5^{\text {th }}$ AIAA Aerodynamic Measurement Technology and Ground Testing Conference, San Francisco, CA.

Lick, W., J. Lick, L. Jin, and J. Gailani. 2005. Approximate equations for sediment erosion rates. Proceedings in Marine Science 8:109-127.

Maa, P.-Y., L. Wright, C. Lee, and T. Shannon. 1993. VIMS Sea Carousel: A field instrument for studying sediment transport. Marine Geology 115(3):271-287. 
McNeil, J., C. Taylor, and W. Lick. 1996. Measurements of the erosion of undisturbed bottom sediments with depth. Journal of Hydraulic Engineering 122(6):316324 .

Muntges, D. E., and J. Majdalani. 2002. Pulsatory channel flow for an arbitrary volumetric flowrate, AIAA 2002-2856. $3^{\text {nd }}$ AIAA Fluid Dynamics Conference. St. Louis. MO.

Oweis, G. F., E. S. Winkel, J. M. Cutbrith, S. L. Ceccio, M. Perlin, and D. R. Dowling. 2010. The mean velocity profile of a smooth-flat-plate turbulent boundary layer at high Reynolds number. Journal of Fluid Mechanics 665(Dec):357-381.

Parchure, T., and A. Mehta. 1985. Erosion of soft cohesive sediment deposits. Journal of Hydraulic Engineering 111(10):1308-26

Raffel, M., C. Willert, and J. Kompenhans. 2002. Particle image velocimetry: A practical guide. Berlin: Springer.

Ray, S., B. Ünsal, F. Durst, Ö. Ertunc, and O. A. Bayoumi. 2005. Mass flow rate controlled fully developed laminar pulsating pipe flows. Journal of Fluids Engineering 127(3):405-418.

Roberts, J., R. Jepsen, D. Gotthard, and W. Lick. 1998. Effects of particle size and bulk density on erosion of quartz particles. Journal of Hydraulic Engineering 124(12):1261-1267.

Roberts, J., R. Jepsen, and S. James. 2003. Measurements of sediment erosion and transport with the adjustable shear stress erosion and transport flume. Journal of Hydraulic Engineering 129(11):862-871.

Salon, S., V. Armenio, and A. Crise. 2007. A numerical investigation of the Stokes boundary layer in the turbulent regime. Journal of Fluid Mechanics 570:253296.

Sarpkaya, T. 1993. Coherent structures in oscillatory boundary layers. Journal of Fluid Mechanics 253:105-140.

Scotti, A., and U. Piomelli. 2001. Numerical simulation of pulsating turbulent channel flow. Physics of Fluids 13(5):1367-1384.

Swart, D. H. 1976. Predictive equations regarding coastal transport. In Proceedings of the $15^{\text {th }}$ Coastal Engineering Conference, ASCE, 1113-1132. New York: American Society of Civil Engineers.

Tsai, C. H., and W. Lick. 1986. A portable device for measuring sediment resuspension. Journal of Great Lakes Research 12(4):314-321.

Uchida, S. 1956. The pulsating viscous flow superposed on the steady laminar motion of incompressible fluid in a circular pipe. Zeitschrift für Angewandte Mathematik und Physik (ZAMP) 7:403-422.

U.S. Army Engineer Waterways Experiment Station. 1998. LTFATE cohesive sediment transport model. Technical Note DOER-N1. Vicksburg, MS: U.S. Army Engineer Waterways Experiment Station. 
White, F. M. 1991. Viscous fluid flow, $2^{\text {nd }}$ ed. New York: McGraw-Hill.

Wikramanayake, P. N., and O. Madsen. 1994. Calculation of suspended sediment transport by combined wave-current flows. Contract Report DRP-94-7. Vicksburg, MS: U.S. Army Engineer Waterway Experiment Station. 


\section{Appendix A: Compilation of Shear Stress Data}

Figure A1. Shear-stress results for operating condition 50_5_45_on.

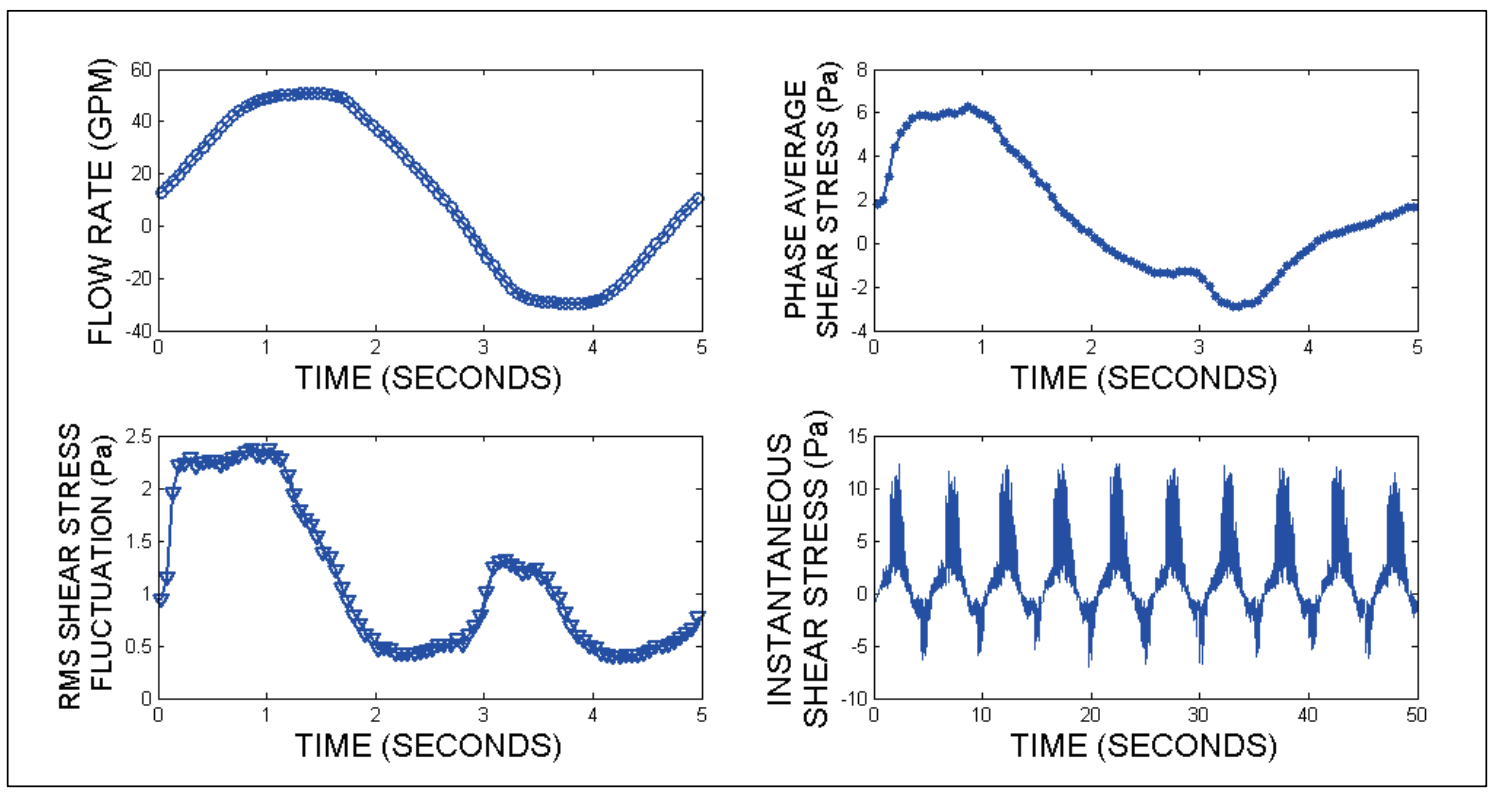

Figure A2. Shear-stress results for operating condition 50_5_60_on.

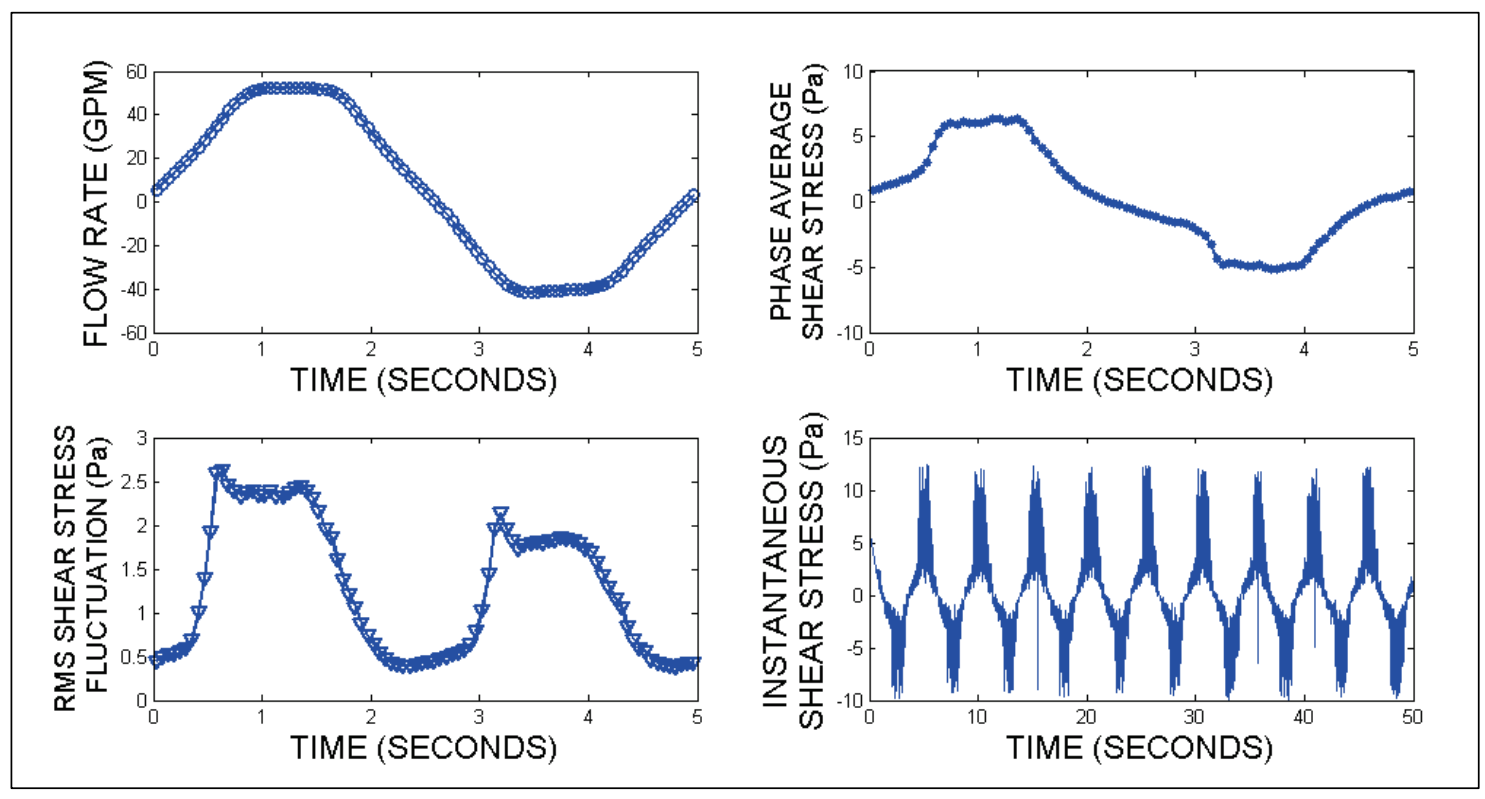


Figure A3. Shear-stress results for operating condition 50_5_open_off.

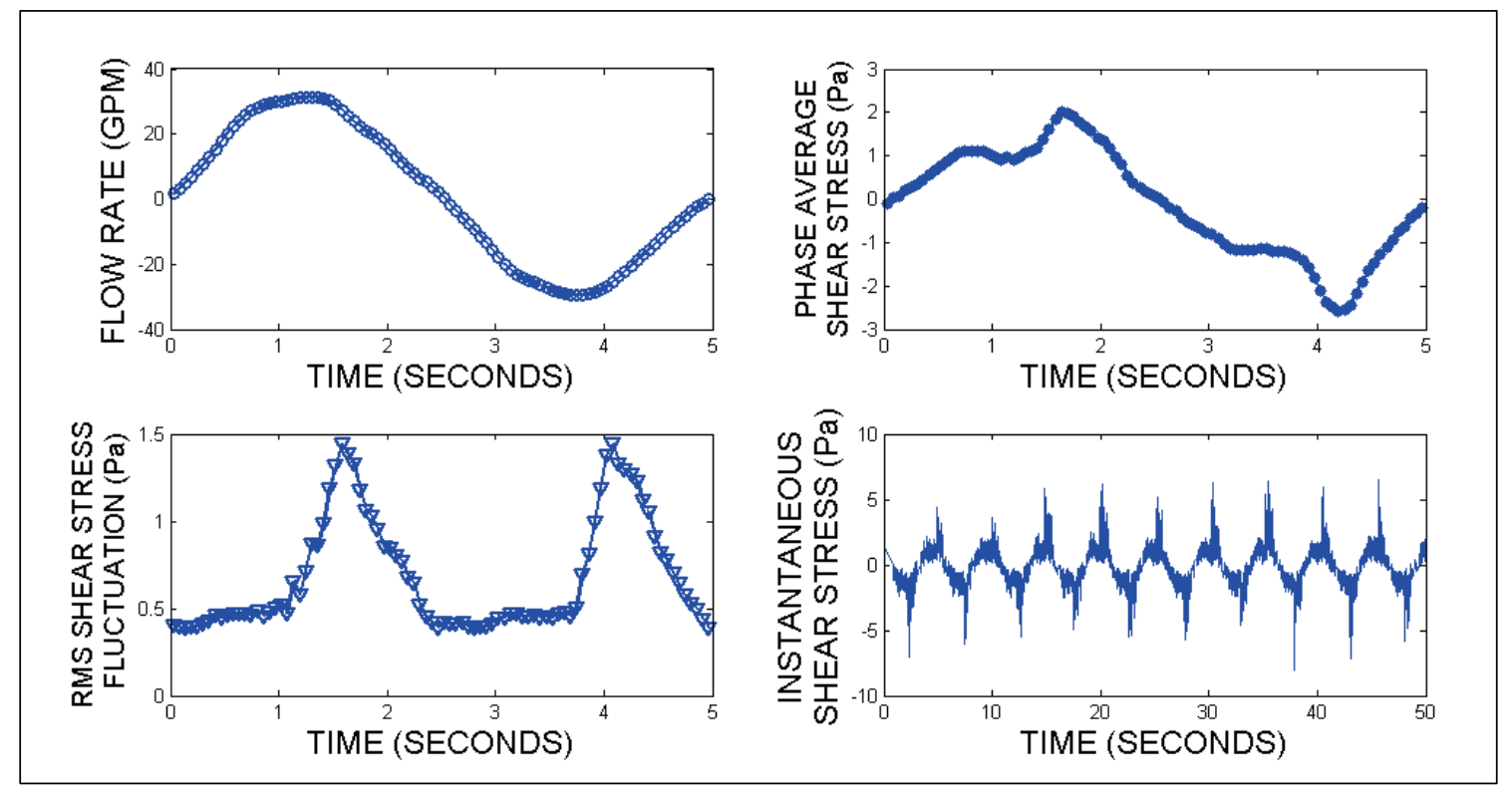

Figure A4. Shear-stress results for operating condition 50_5_45_off.

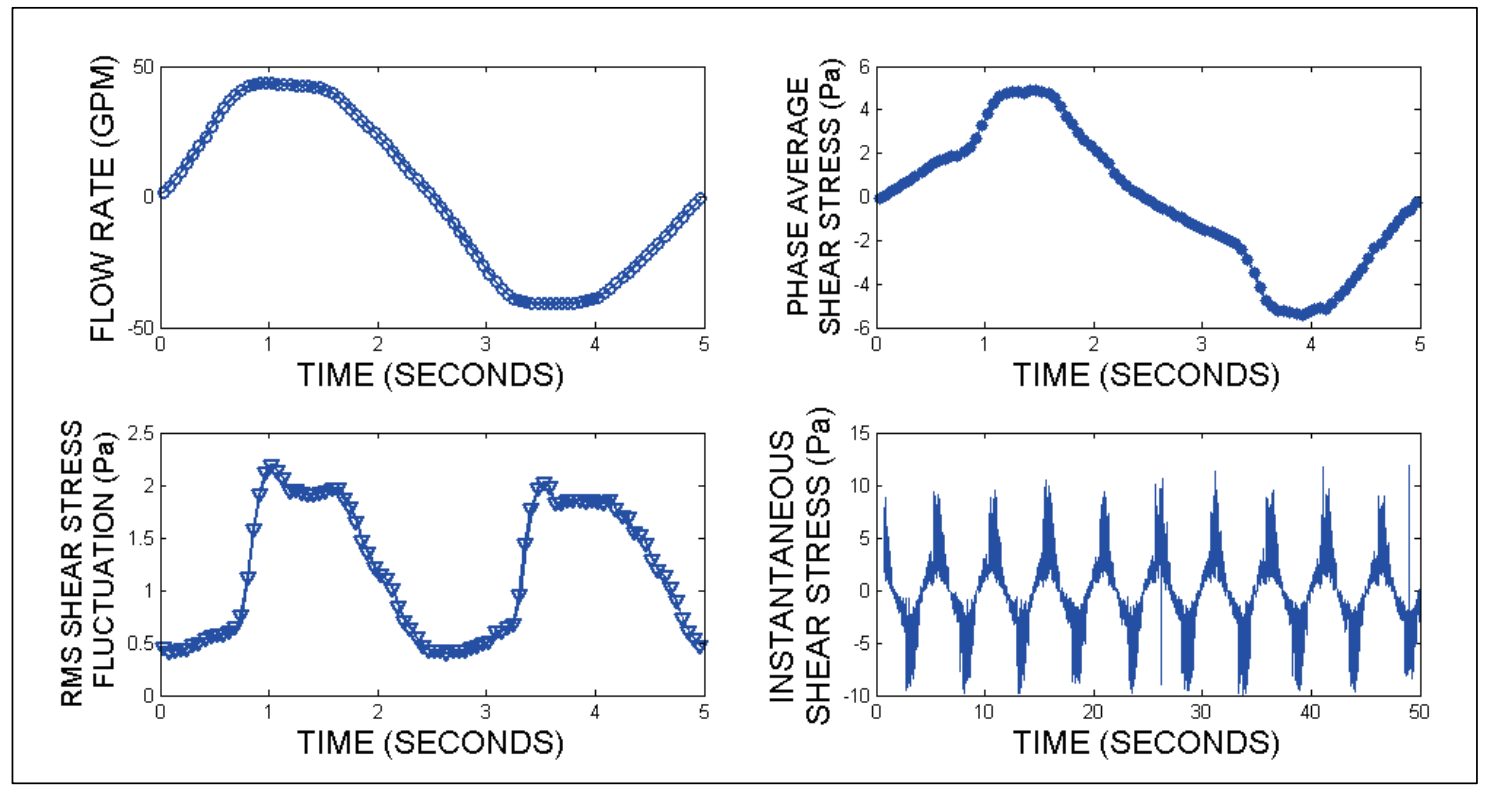


Figure A5. Shear-stress results for operating condition 50_5_60_off.

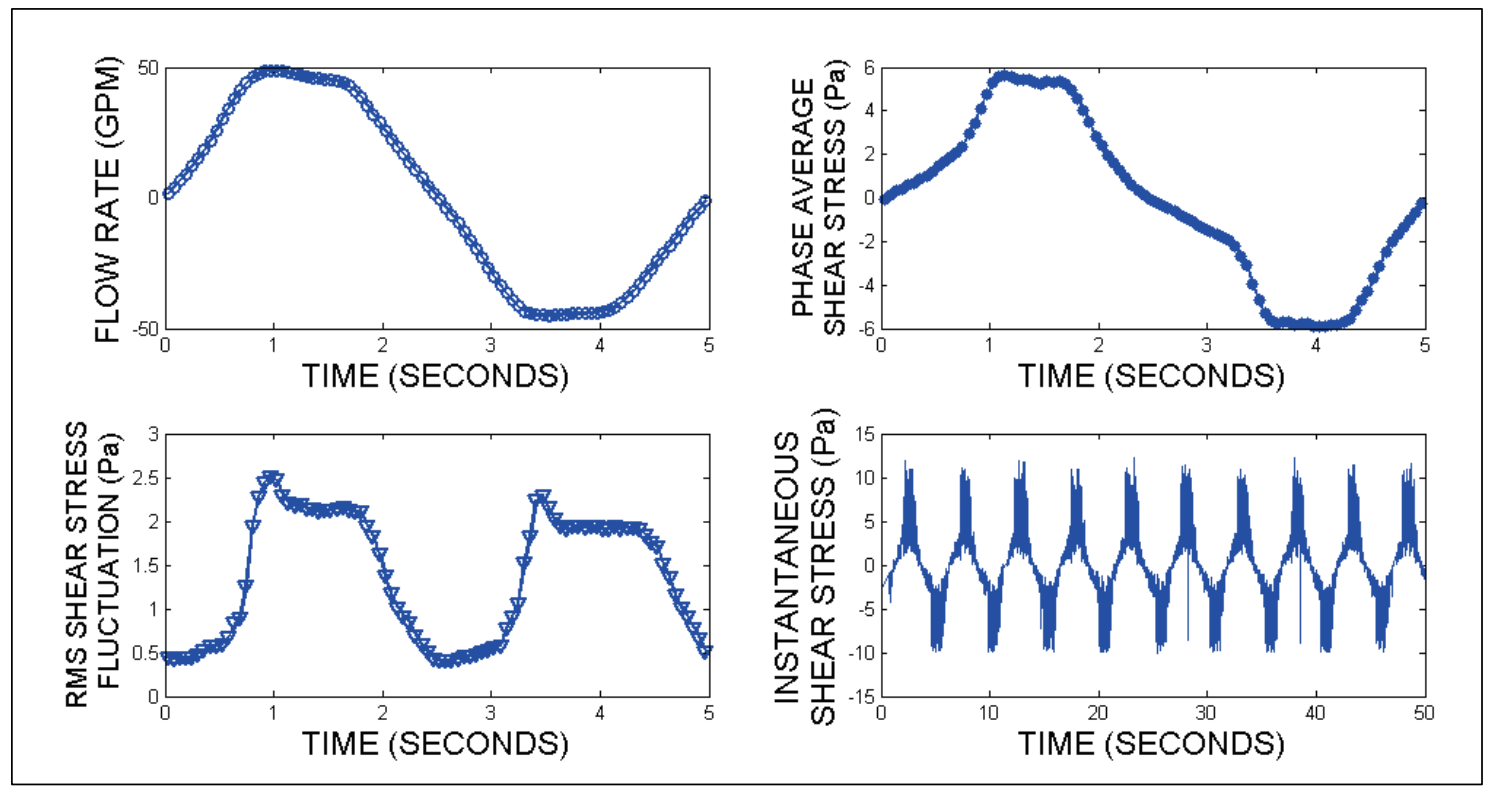

Figure A6. Shear-stress results for operating condition 50_10_open_on.

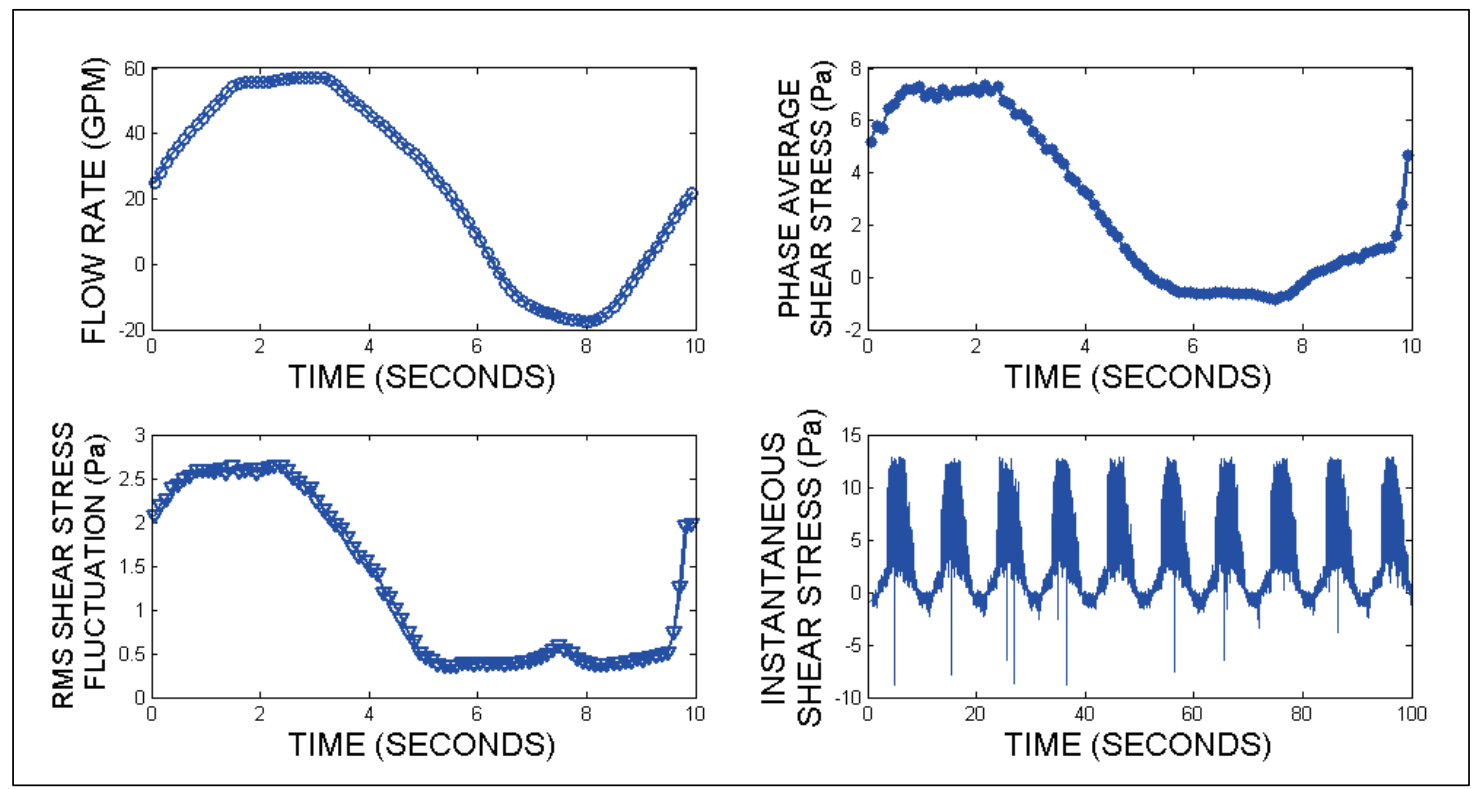


Figure A7. Shear-stress results for operating condition 50_10_45_on.

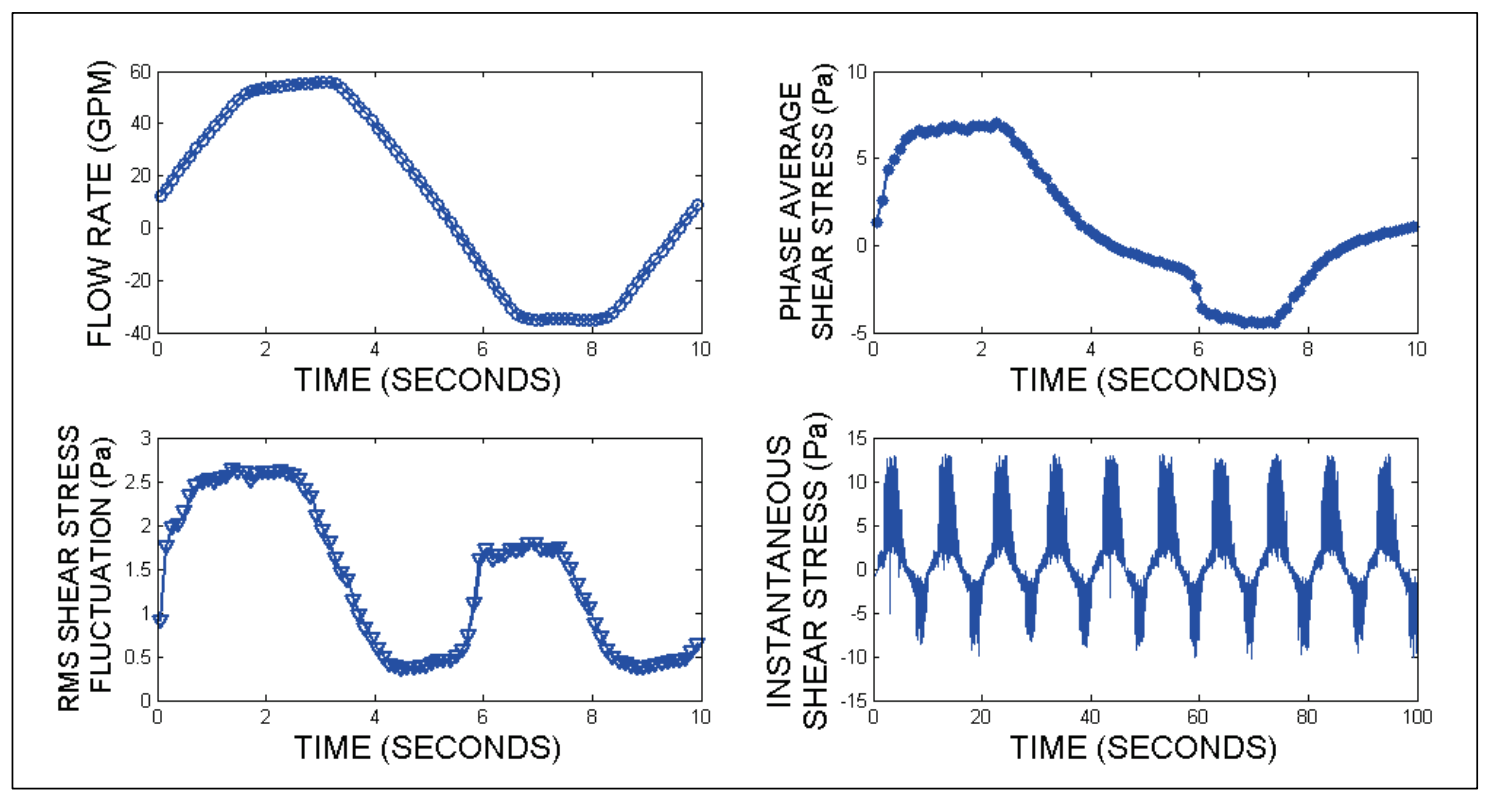

Figure A8. Shear-stress results for operating condition 50_10_open_off.

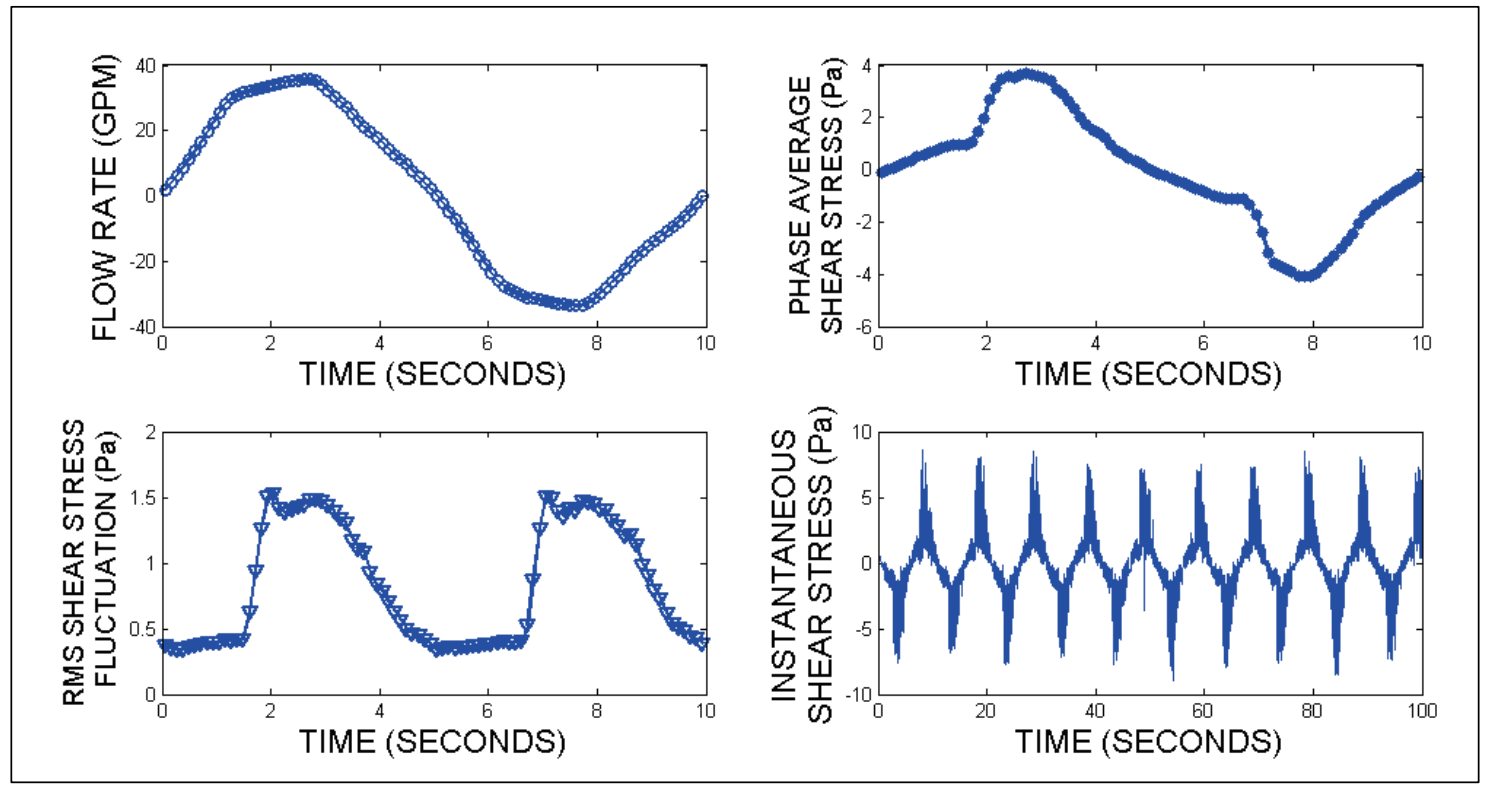


Figure A9. Shear-stress results for operating condition 50_10_45_off.

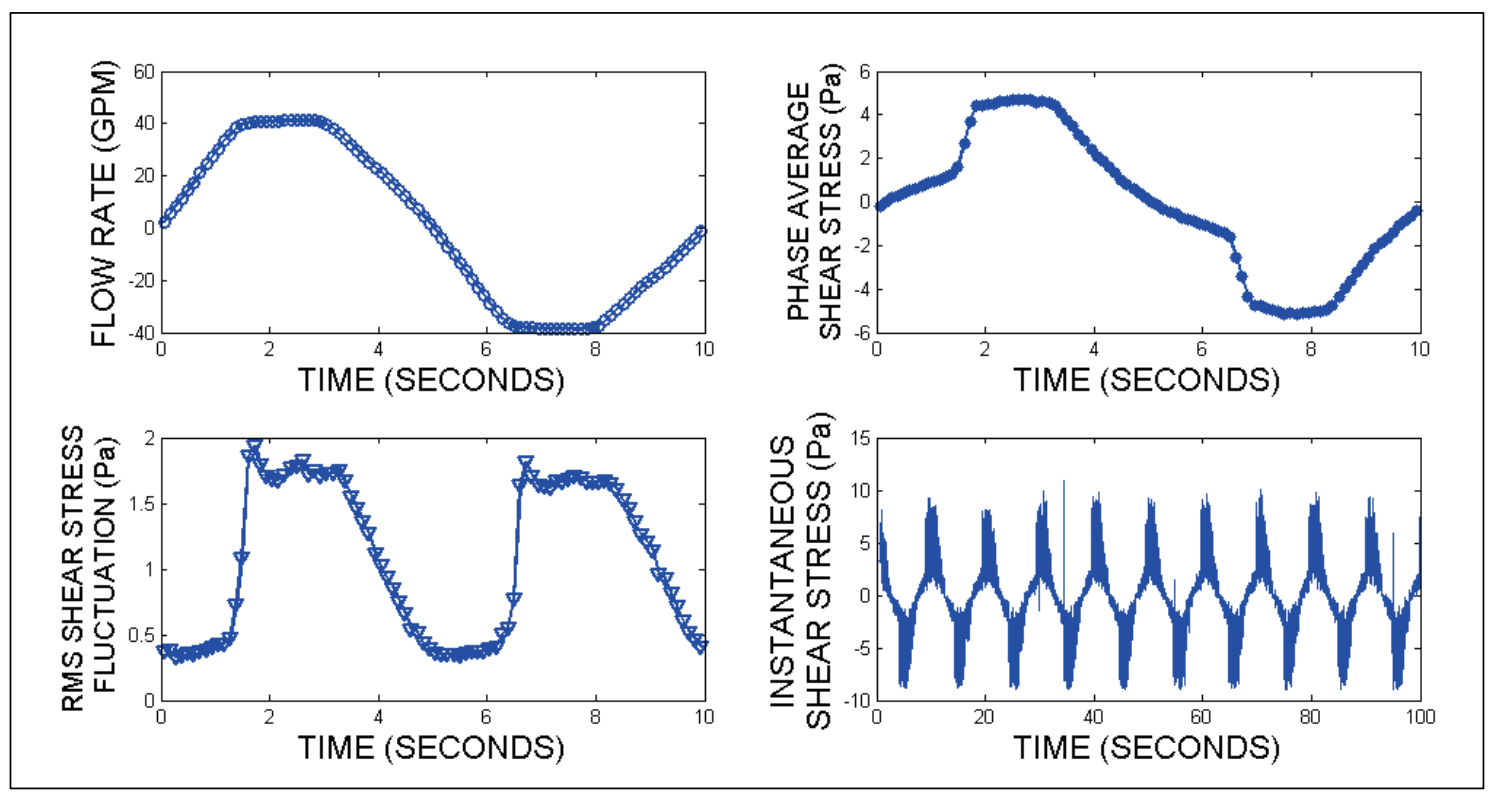

Figure A10. Shear-stress results for operating condition 50_10_60_off.

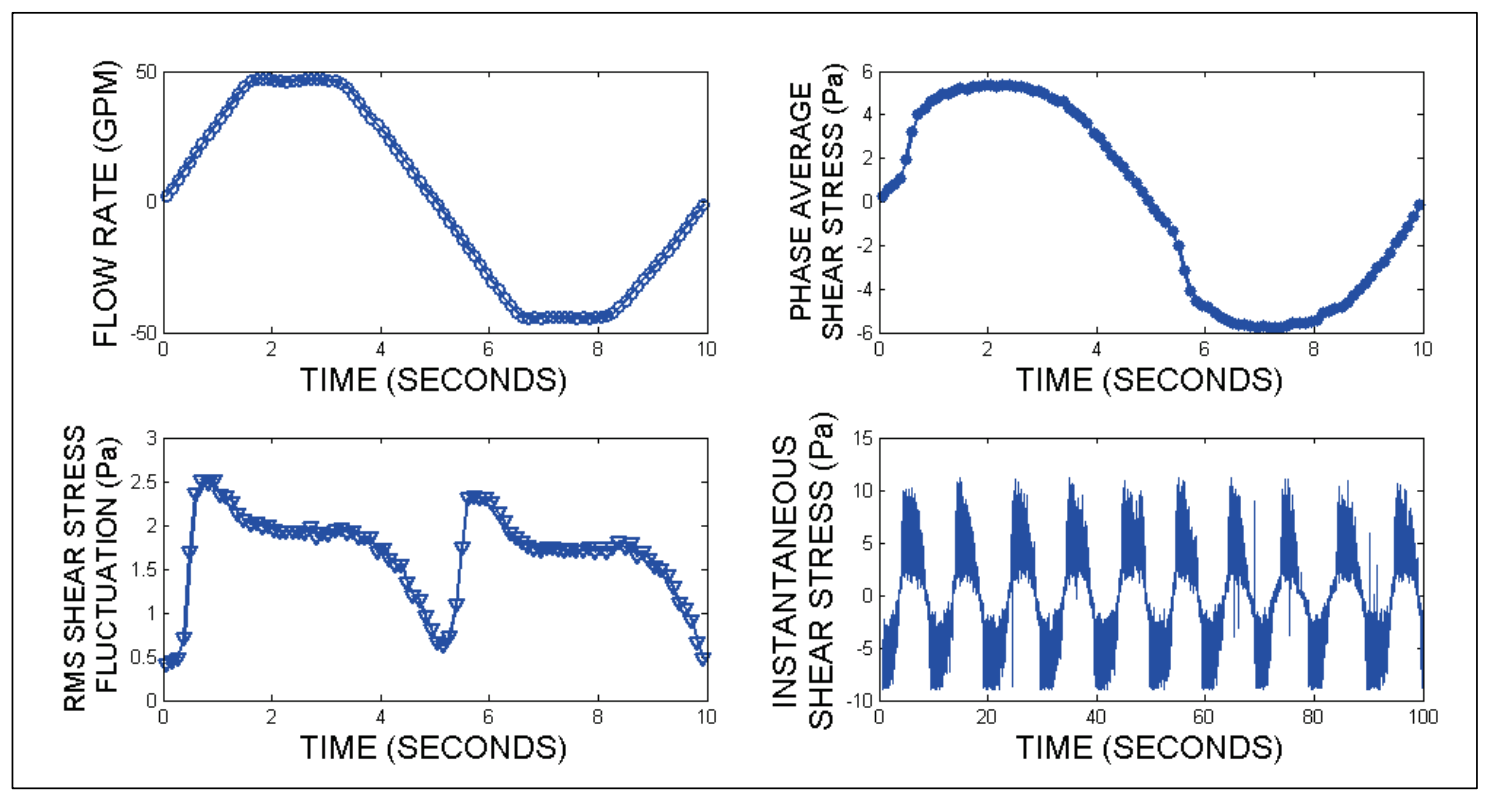


Figure A11. Shear-stress results for operating condition 40_10_45_off.

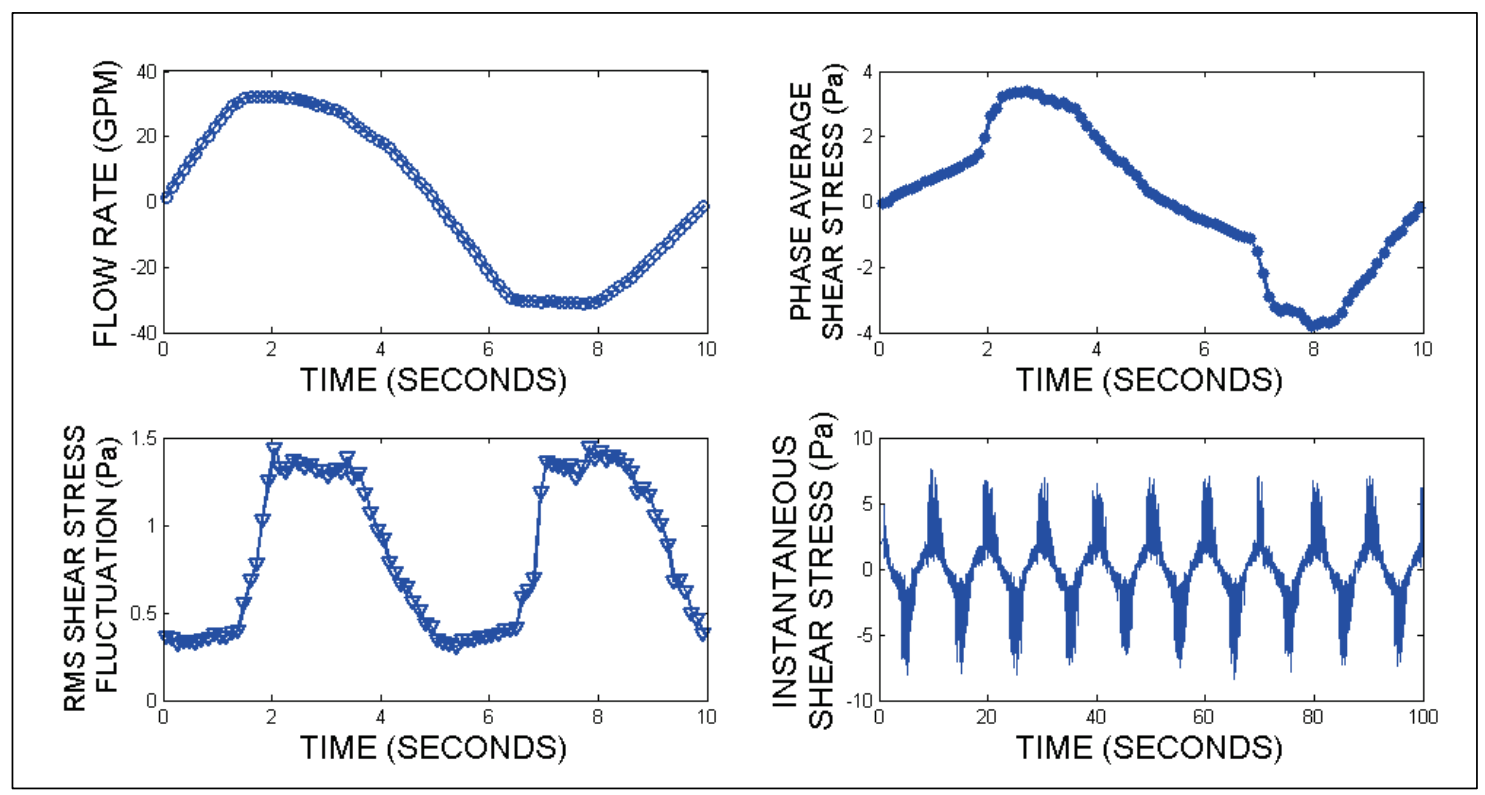

Figure A12. Shear-stress results for operating condition 40_10_45_on.

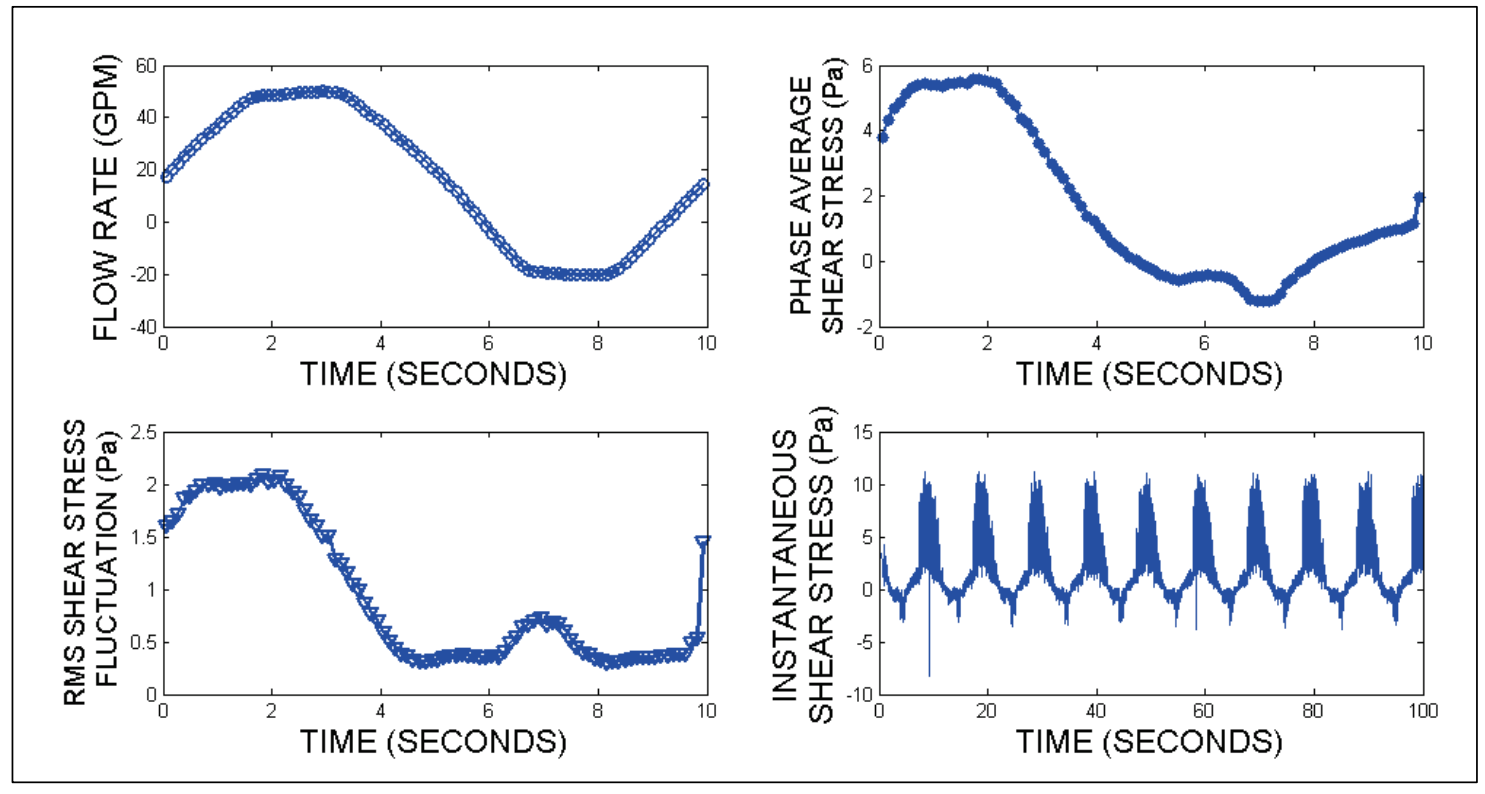


Figure A13. Shear-stress results for operating condition 40_10_60_on.

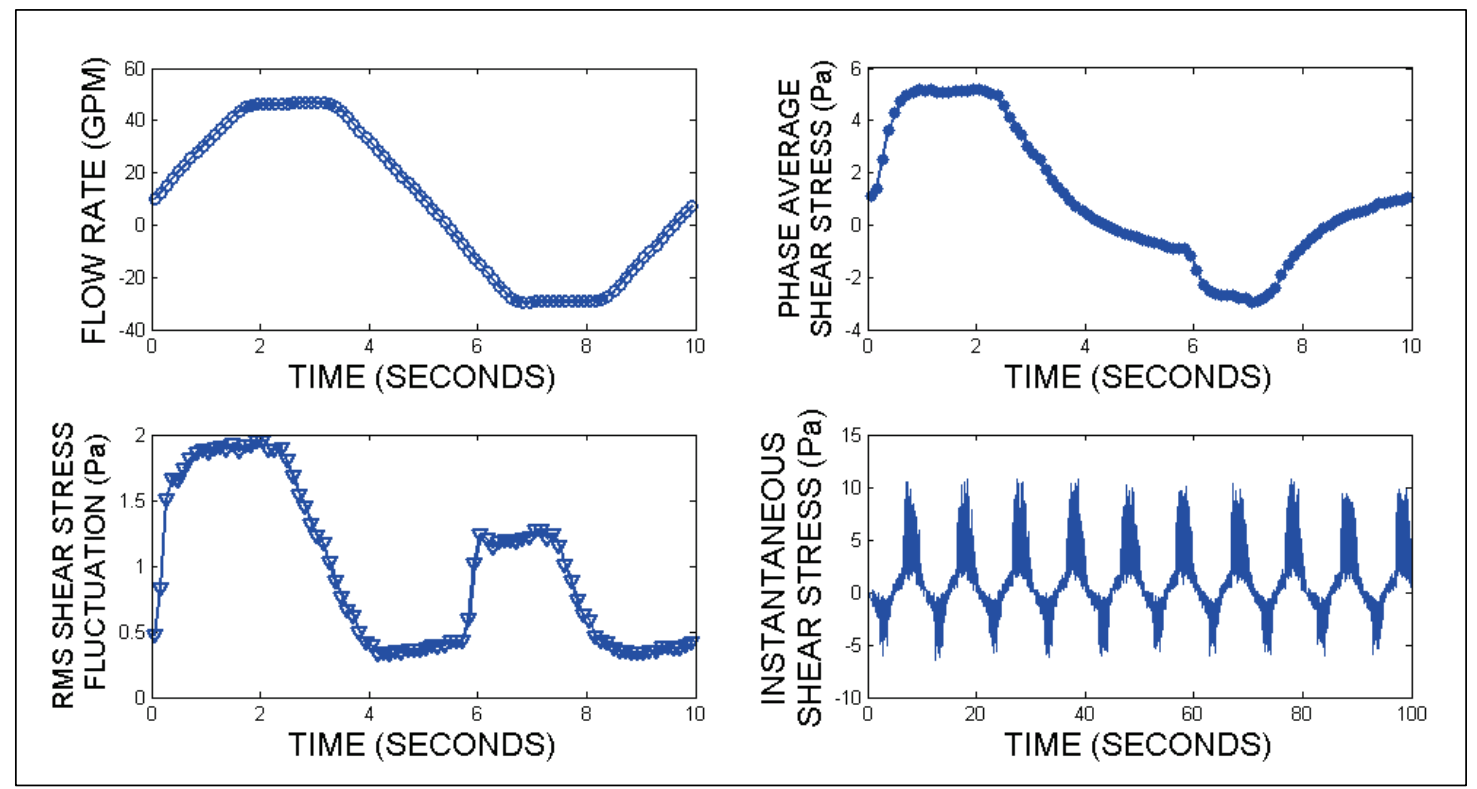

Figure A14. Shear-stress results for operating condition 40_10_open_off.

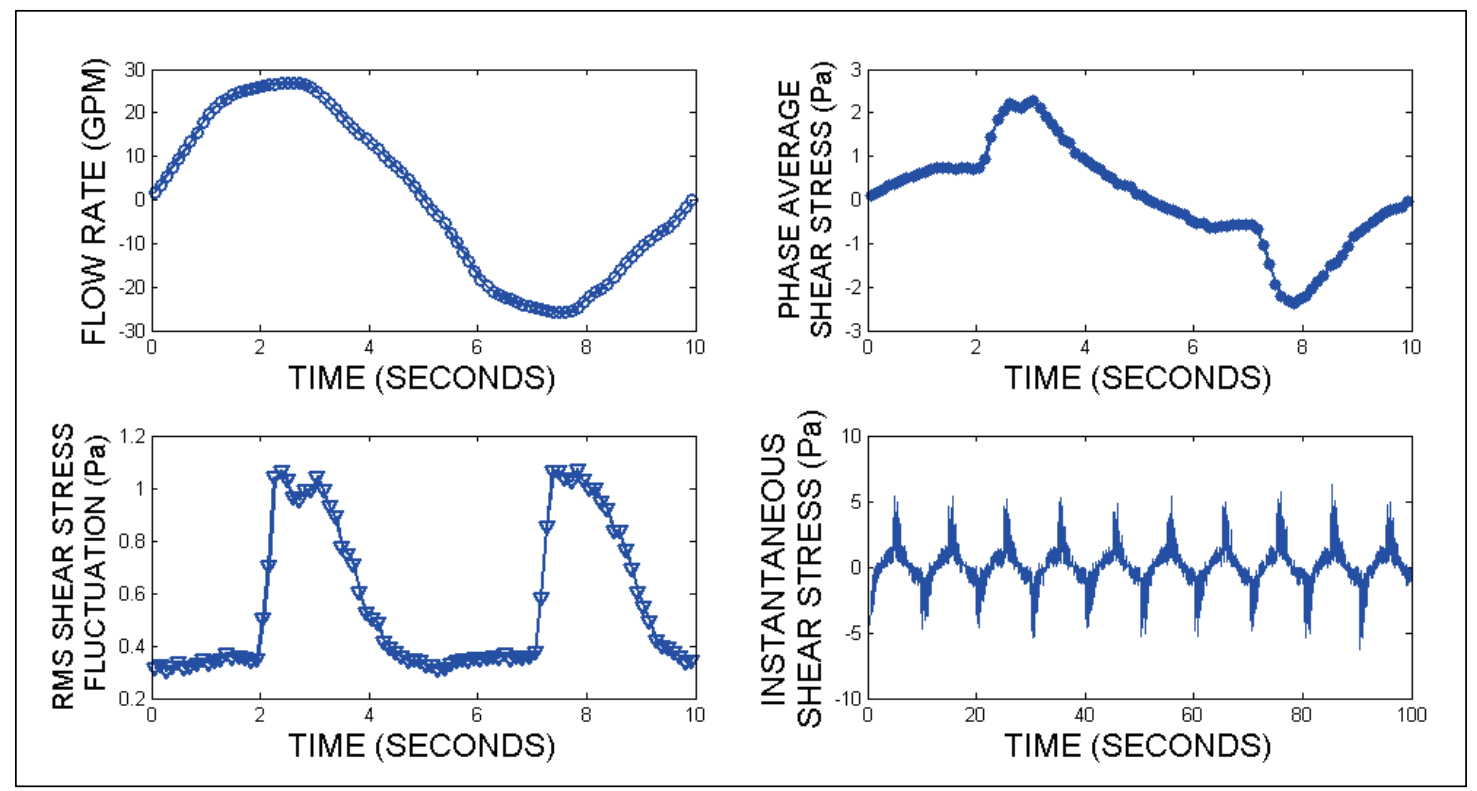


Figure A15. Shear-stress results for operating condition 40_10_open_on.

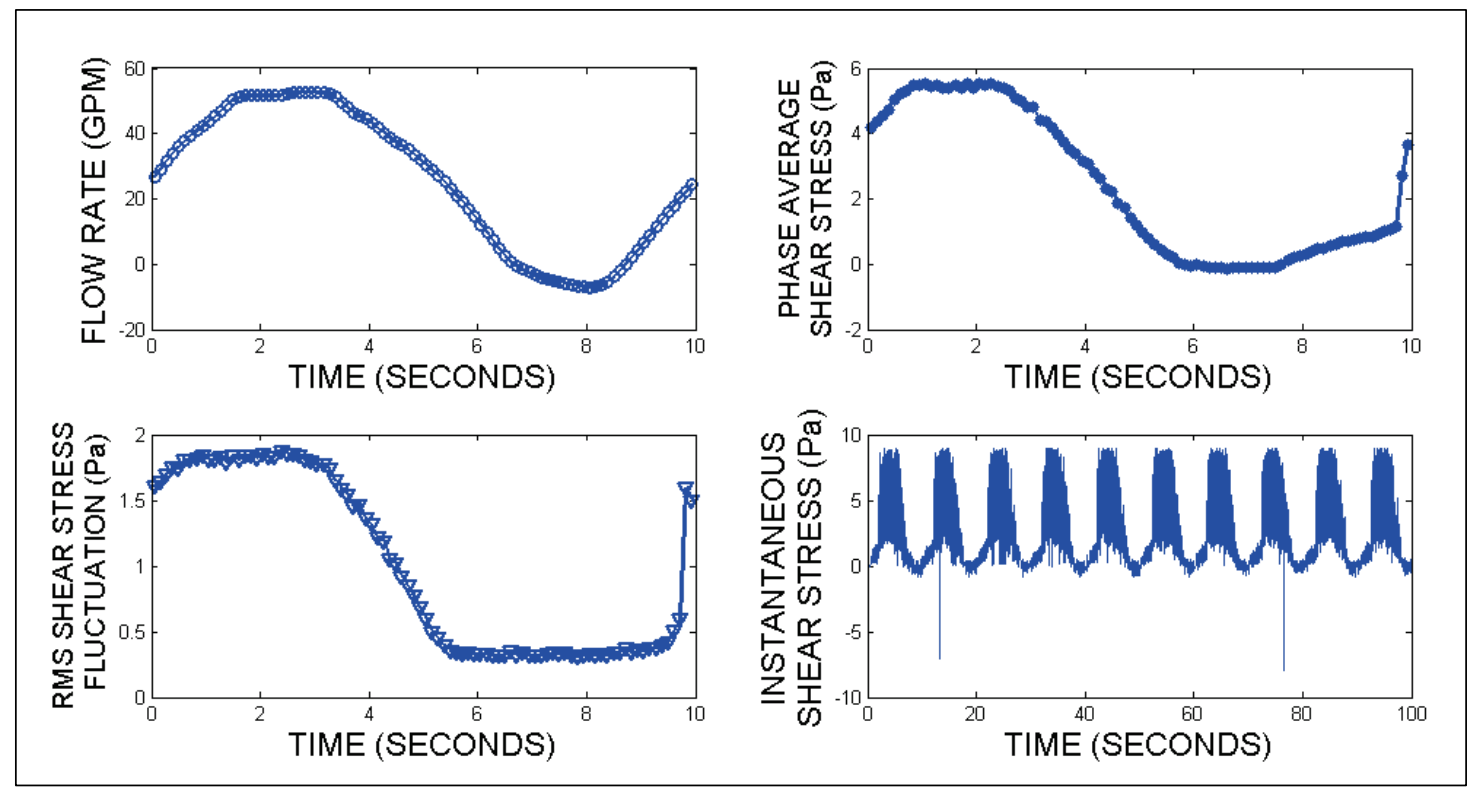

Figure A16. Shear-stress results for operating condition 40_5_open_on.

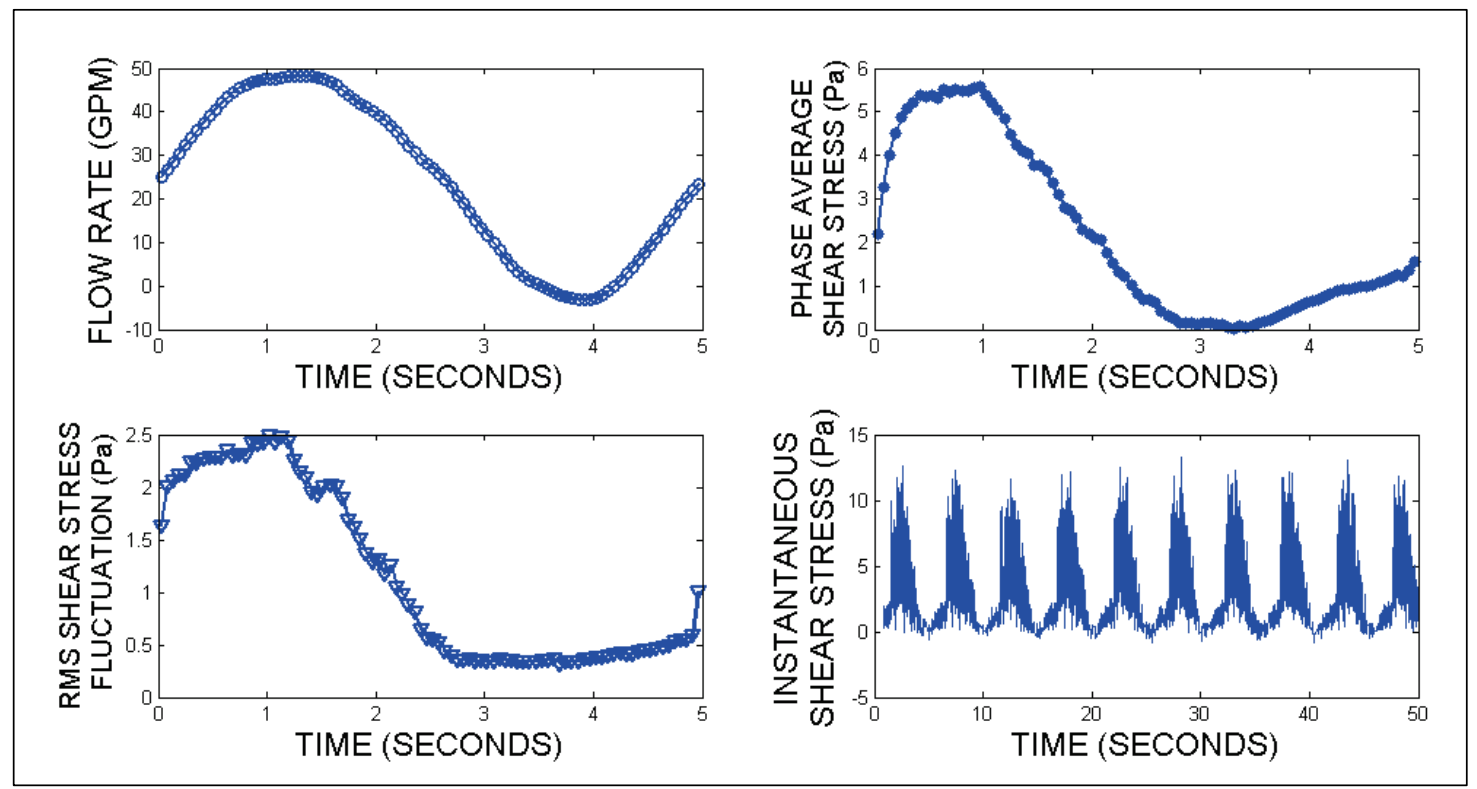


Figure A17. Shear-stress results for operating condition 40_5_45_on.

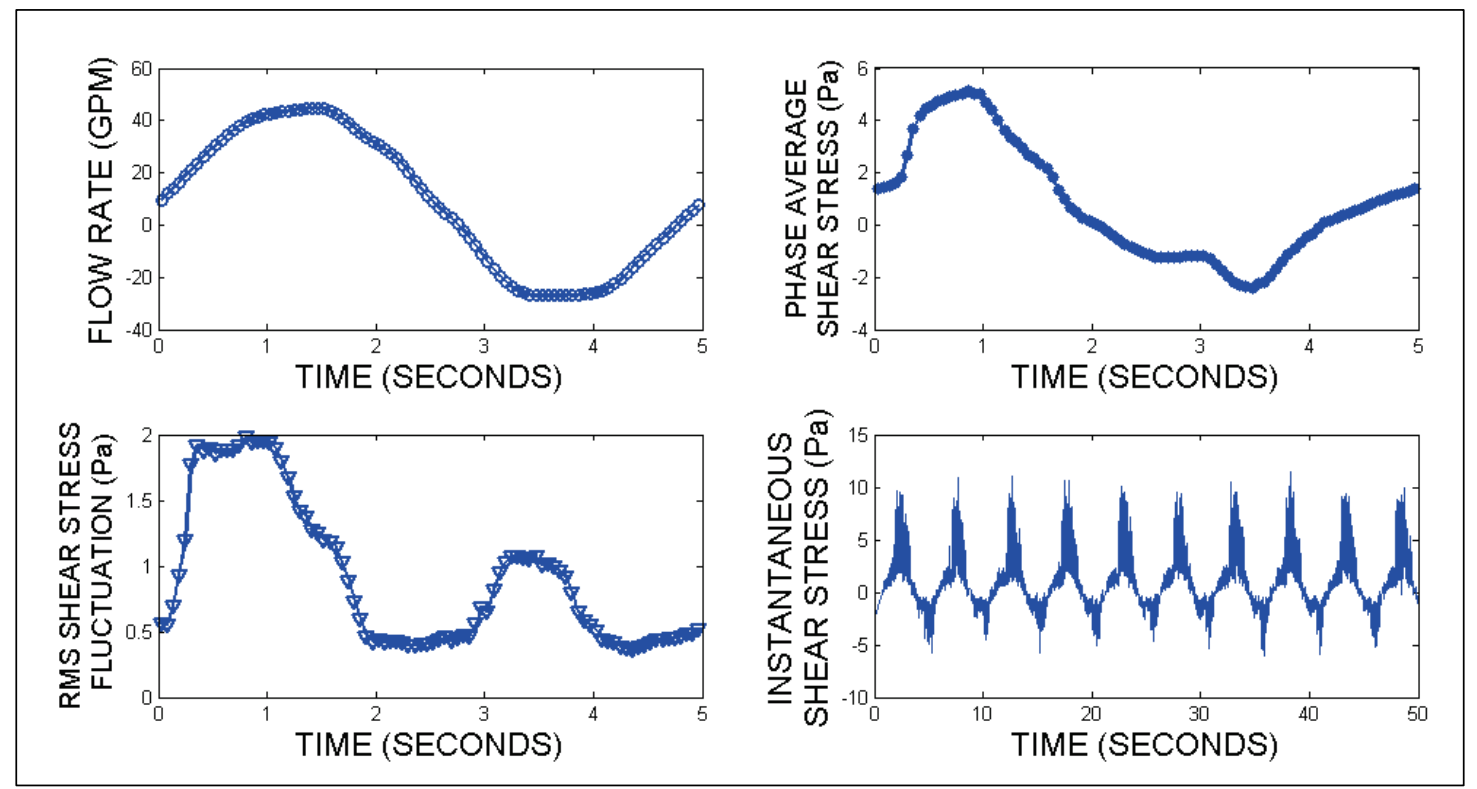

Figure A18. Shear-stress results for operating condition 40_5_60_on.

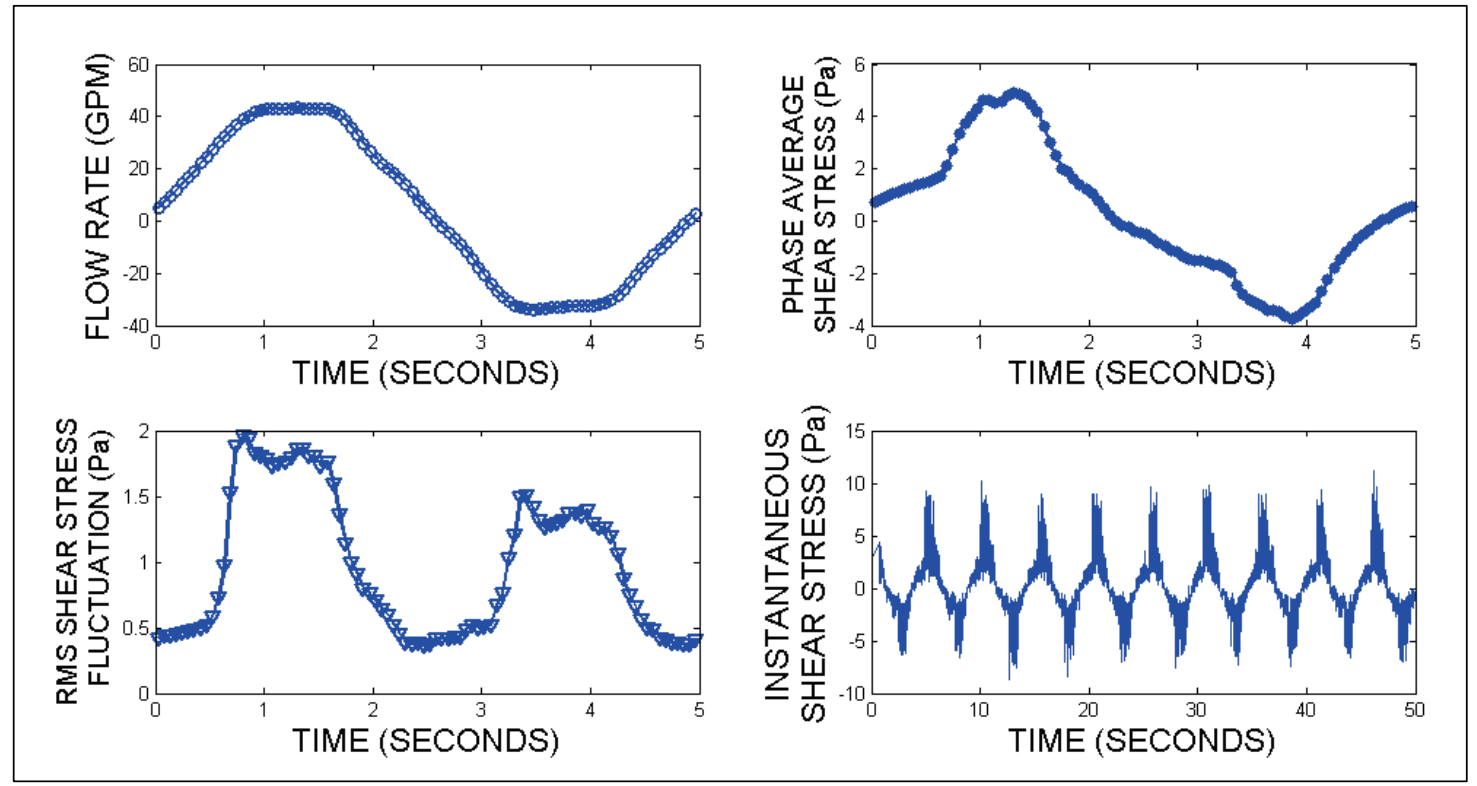


Figure A19. Shear-stress results for operating condition 40_5_open_off.

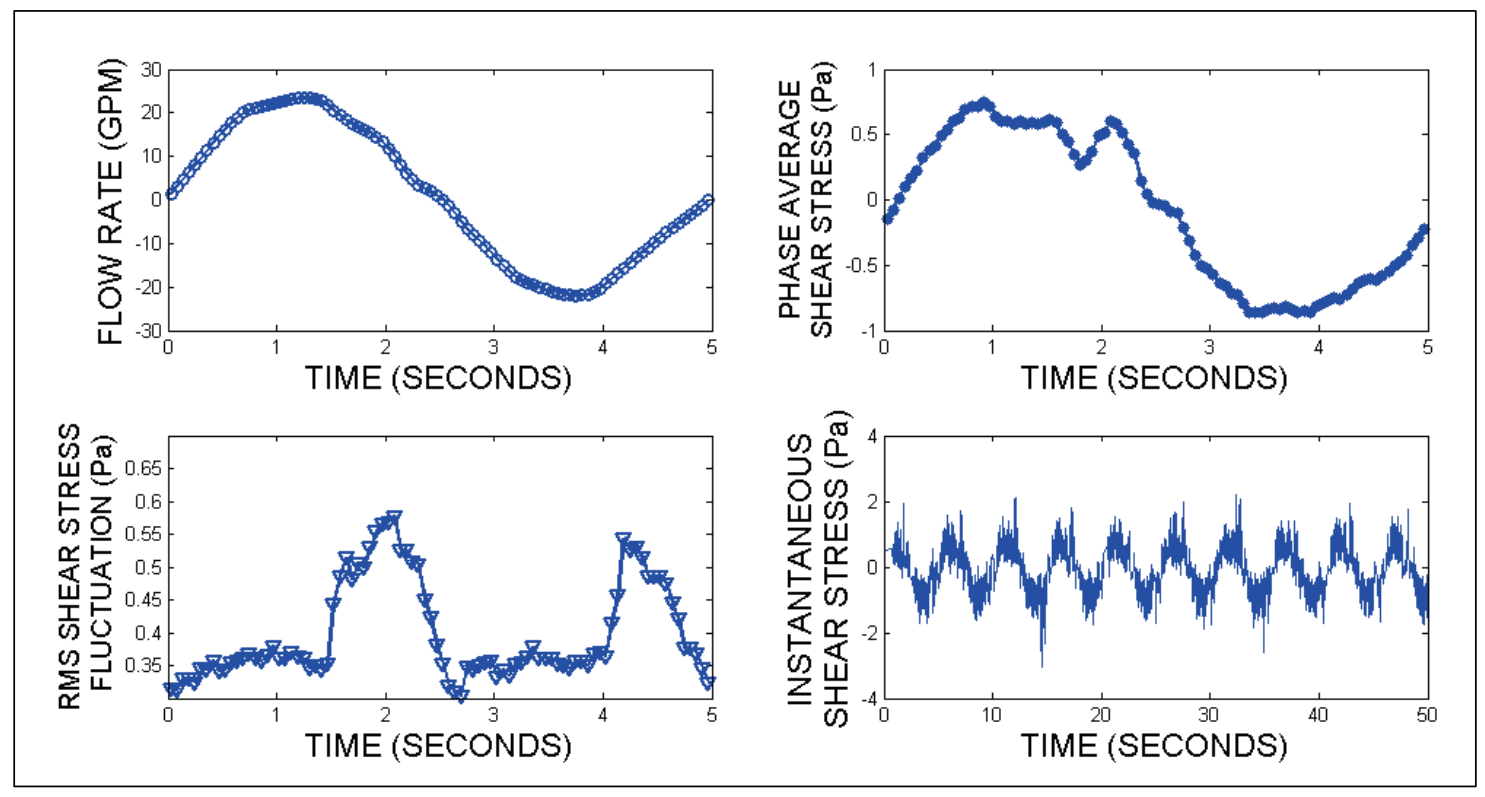

Figure A20. Shear-stress results for operating condition 40_5_45_off.

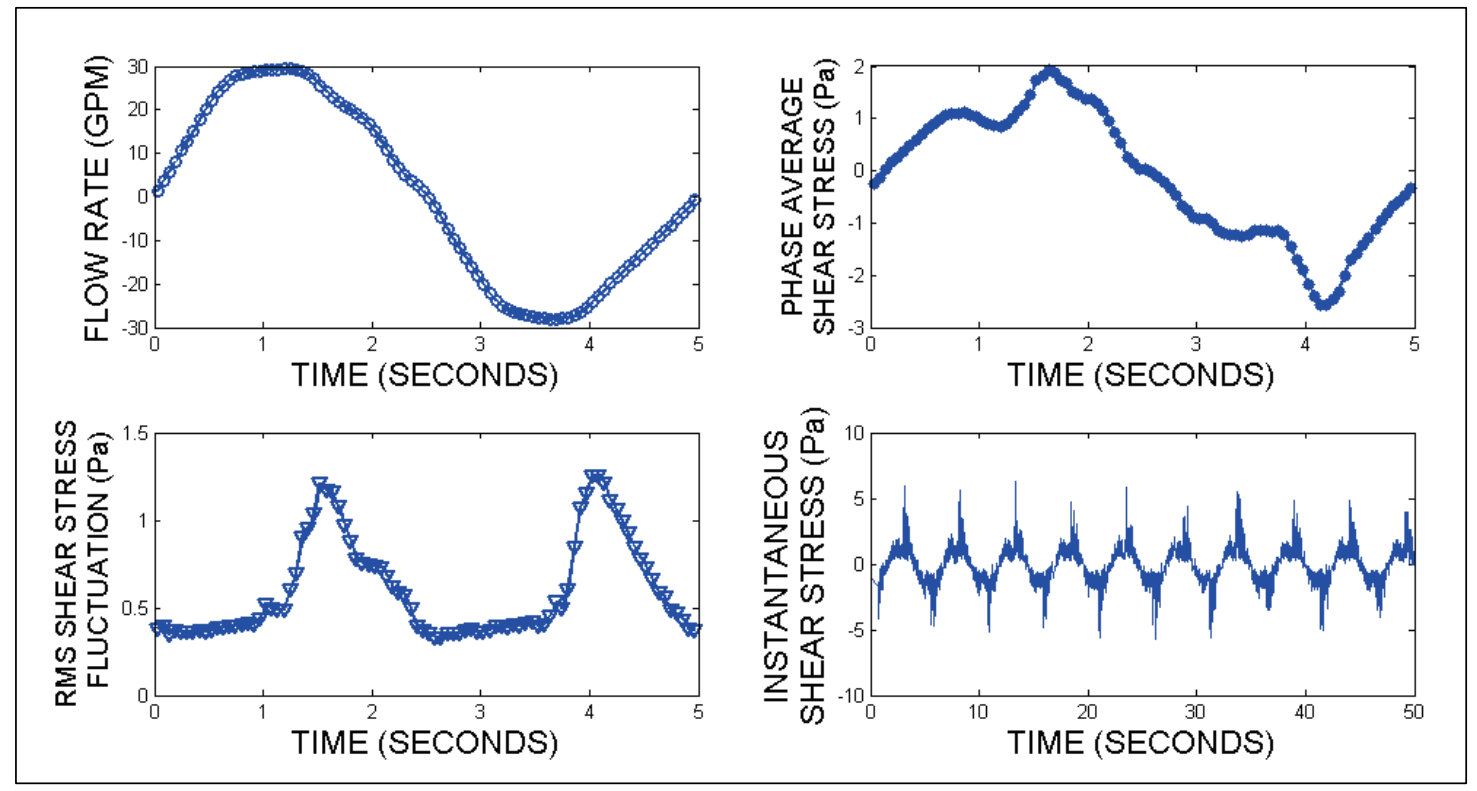


Figure A21. Shear-stress results for operating condition 40_5_60_off.

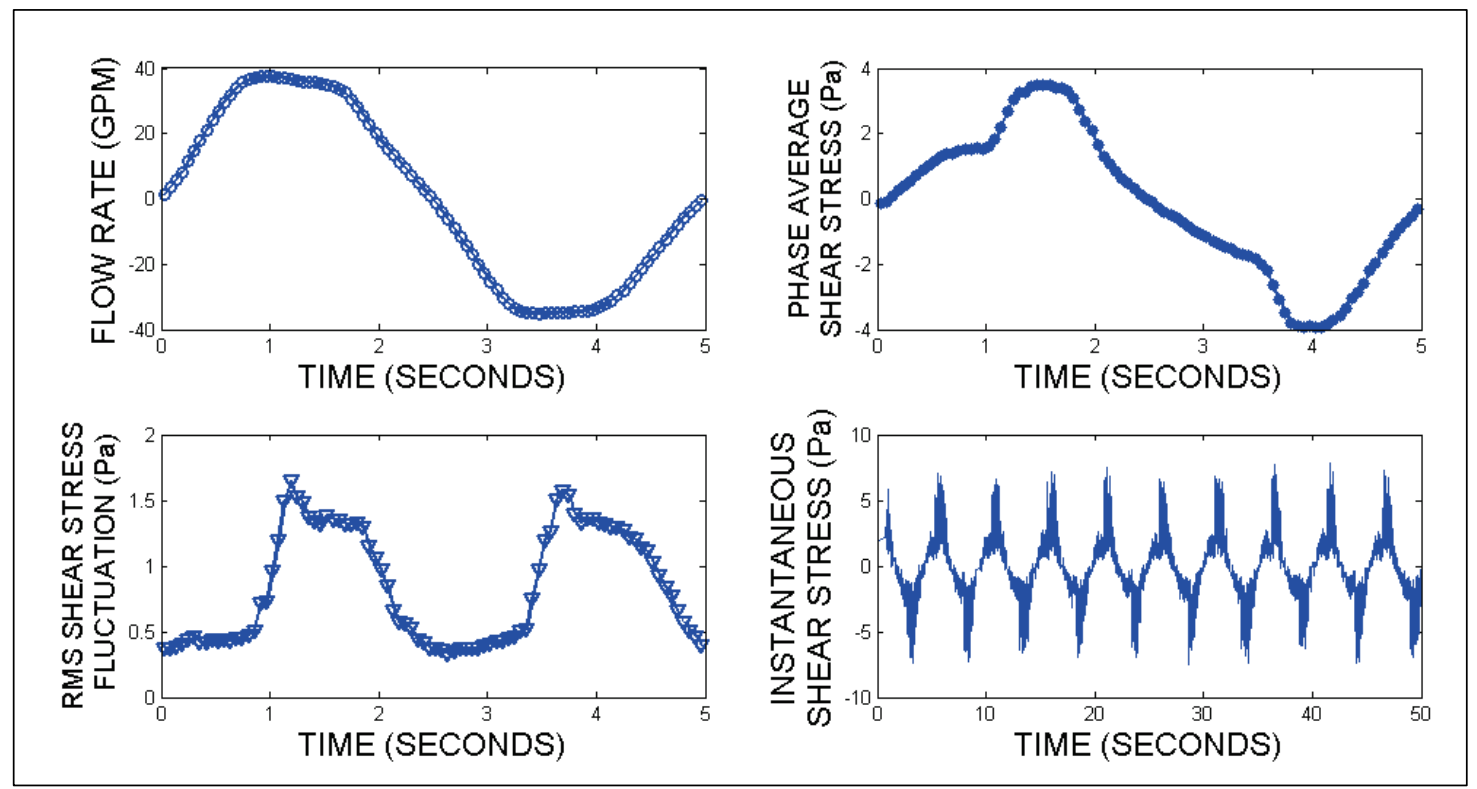

Figure A22. Shear-stress results for operating condition 50_5_open_on.

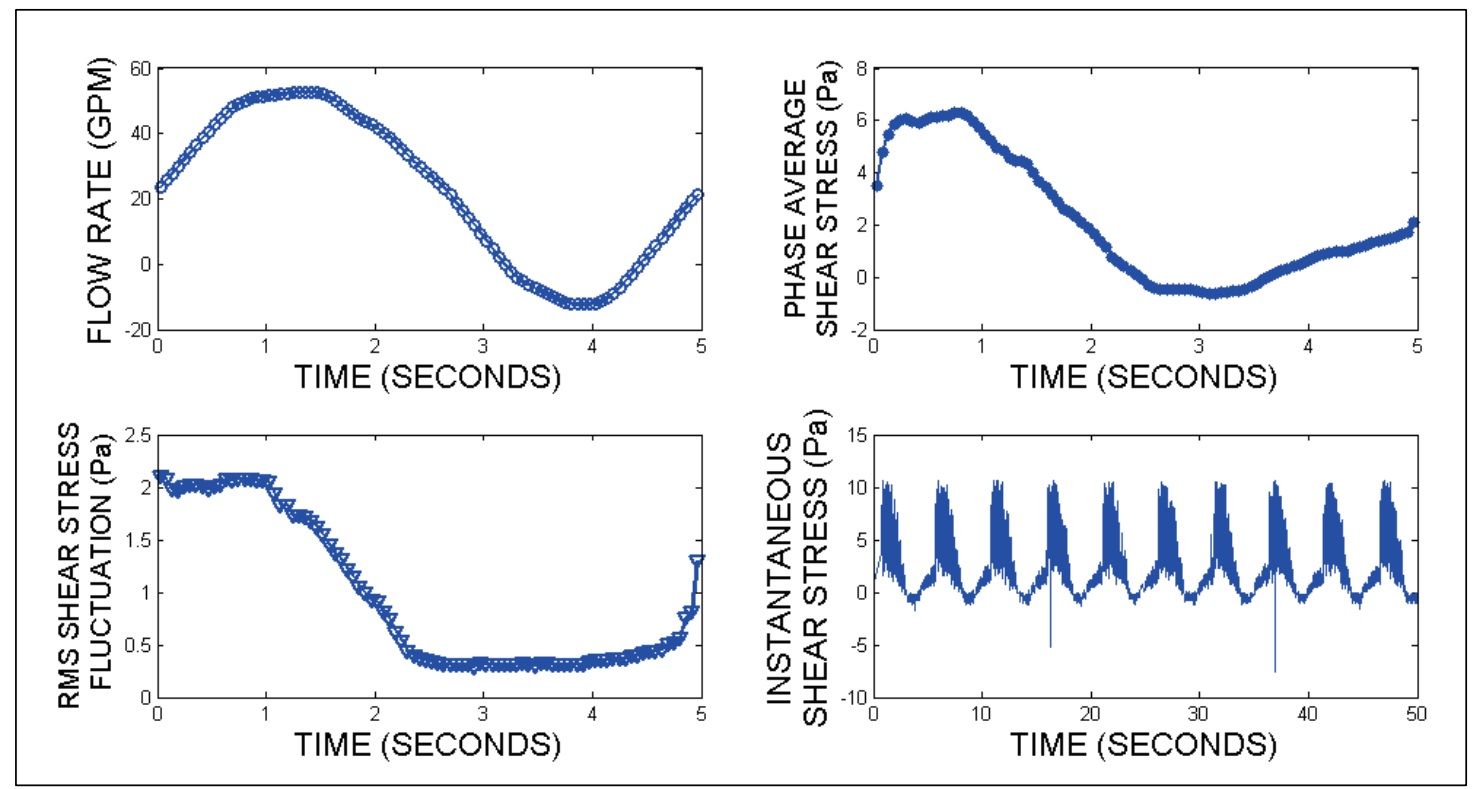




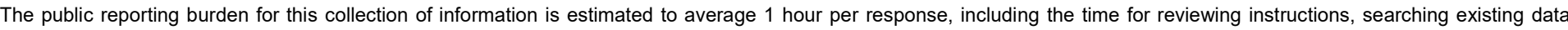

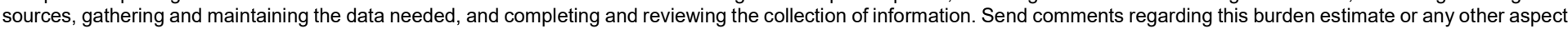

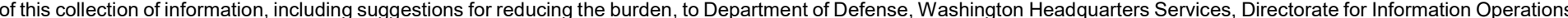

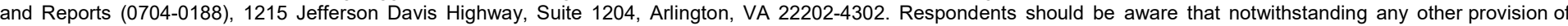
law, no person shall be subject to any penalty for failing to comply with a collection of information if it does not display a currently valid OMB control number. PLEASE DO NOT RETURN YOUR FORM TO THE ABOVE ADDRESS.

\section{REPORT DATE \\ September 2017 \\ 2. REPORT TYPE \\ Final Report}

\section{TITLE AND SUBTITLE}

Develop Accurate Methods for Characterizing And Quantifying Cohesive Sediment

Erosion Under Combined Current-Wave Conditions: Project ER 1497
6. AUTHOR(S)

Joseph Z. Gailani, Jesse D. Roberts, Sean P. Kearny

\section{DATES COVERED (From - To)}

\section{5a. CONTRACT NUMBER}

5b. GRANT NUMBER

5c. PROGRAM ELEMENT NUMBER $622784 \mathrm{~T} 4000$

5d. PROJECT NUMBER

5e. TASK NUMBER

5f. WORK UNIT NUMBER

W74RDV51808301

\section{PERFORMING ORGANIZATION} REPORT NUMBER

ERDC/CHL TR-17-15

10. SPONSOR/MONITOR'S ACRONYM(S) SERDP

11. SPONSOR/MONITOR'S REPORT NUMBER(S)
ERDC, 3909 Halls Ferry Road, Vicksburg, MS 39180

Sandia National Laboratories, 1515 Eubank SE, Albuquerque, NM 87123

\section{SPONSORING/MONITORING AGENCY NAME(S) AND ADDRESS(ES)}

Strategic Environmental Research and Development Program (SERDP), 48000 Mark

Center Drive, Suite 17D08, Alexandria, VA 22350-3605

\section{DISTRIBUTION/AVAILABILITY STATEMENT}

Approved for public release; distribution is unlimited.

\section{SUPPLEMENTARY NOTES}

\section{ABSTRACT}

Aquatic cohesive sediments are often the final receptor of contaminants released by Department of Defense (DoD) activities. In estuaries, harbors, and channels, fine sediments can be mobilized and transported by the combined action of both relatively steady currents (forced by river flow, tides, and wind) and by the larger unsteady bottom shear stresses associated with higher-frequency oscillatory wave motions (either wind- or vessel-generated waves). Methods to measure contaminated sediment stability and erosion as influenced by wave processes are critical to assessing contaminant fate and associated risk as well as evaluating remediation options. This research focused on calibrating and verifying the applicability of a prototype flume designed to measure erosion of fine-grained cohesive sediment under combined unidirectional and superimposed oscillatory bottom shear stress. These are similar to the shear-stress conditions that exist in wave-current environments. This research will provide more accurate methods for assessing contaminated sediment stability for many DoD and Environmental Protection Agency managed contaminated sediment sites.

\section{SUBJECT TERMS}

Bed Shear Stress, Bottom Boundary Layer, Cohesive Sediment, Oscillatory Flow, Particle Image Velocimetry, Sediment Transport, Waves and currents,

\section{SECURITY CLASSIFICATION OF:}

\begin{tabular}{|l|l|l|}
\hline a. REPORT & b. ABSTRACT & c. THIS PAGE \\
Unclassified & Unclassified & Unclassified
\end{tabular}

17. LIMITATION OF ABSTRACT

SAR

\section{NUMBER OF PAGES}

100 19a. NAME OF RESPONSIBLE PERSON Joseph Gailani

19b. TELEPHONE NUMBER (Include area code) 601-634-4851 